\title{
30th Meeting of the Canadian Congress of Neurological Sciences
}

\section{ORAL PRESENTATIONS}

Thursday, June 22
A. Neurosurgery - General and Spinal (14:00 - 17:00)
B. Neurology - General (14:00 - 15:15)
A-1 to A- 10
C. Cerebrovascular Disease (15:30 - 17:00)
B-1 to B-4
D. Clinical Neurophysiology $(14: 00-15: 15)$ C- 1 to $\mathrm{C}-6$
E. Movement Disorders (15:30 - 17:00) D- 1 to D-5
F. Neurogenetics (14:00 - 15:15) E-1 to E-6
G. Pediatric Neurology $(15: 30-16: 45)$ $\mathrm{F}-1$ to $\mathrm{F}-5$ G-1 to G-5

Friday, June 23

\section{Morning}

H. Neurosurgery - Trauma (10:30 - 12:00) $\mathrm{H}-1$ to $\mathrm{H}-5$

I. Neurology - General (10:30 - 12:00) I-1 to I-5

J. Clinical Neurophysiology $(10: 30-12: 00)$ $\mathrm{J}-1$ to $\mathrm{J}-5$

K. Pediatric Neurology (10:30 - 12:00) K-1 to K-6

\section{Afternoon}

L. Neurology - General (14:00 - 17:00) L- 1 to L-11

M. Epilepsy (14:00 - 17:00) $\mathrm{M}-1$ to $\mathrm{M}-11$ N. Neurosurgery - Vascular (14:00 - 17:00) $\mathrm{N}-1$ to $\mathrm{N}-11$

O. Neurosurgery - Pediatric (14:00 - 17:00) O-1 to O-11

\section{Oral Presentations}

\section{A. NEUROSURGERY - GENERAL AND SPINAL $(A-I$ to $A-10)$ (Thursday, June 22, 14:00 - 17:00)}

\section{A-1}

Conus Medularis and Low Lumbar Spinal Cord Symptoms Caused by Undetected Remote Dural AVM - a Poorly Recognized Entity

J.F.R. Fleming, M. TAYlor, M.C. Wallace and R.A. WILLINSKY (Toronto, Ontario)

When neurological symptoms and findings point to a lesion in the lumbar spinal cord and conus medularis, neuro-imaging is normally directed to the thoracolumbar spine. Subtle abnormalities in the spinal cord may be missed, or an incidental abnormality, such as a bulging thoracolumbar disc, may wrongly be blamed for the clinical picture when the real culprit is a dural AVM elsewhere in the spinal axis. Venous stasis in the lowest portion of the spinal cord, caused by shunting of arterial blood into the peri-medullary venous system by the dural AVM, is responsible for the neurological findings, and if untreated results in permanent neurological damage. Early diagnosis and prompt

\section{POSTER PRESENTATIONS}

Thursday, June 22

(07:00 - 17:00, Authors Standing By 13:00 - 14:00)

\section{Session I}

$\mathrm{P}-1$ to $\mathrm{P}-13$

$\mathrm{P}-14$ to $\mathrm{P}-21$

Pediatric Neurology

P-22 to P-25 Neurosurgery - Trauma

P-26 to P-49

P-50 to P-54

P-55 to P-59

Neurosurgery - Vascular

P-60 to P-69

P-70 to P-77 Neurology - Vascular Multiple Sclerosis Neuroradiology Peripheral Nerve Movement Disorders

Friday, June 23

(07:00 - 17:00, Authors Standing By 13:00 - 14:00)

Session Il

P-78 to P-90

P-91 to P-102

Epilepsy

P-103 to P-115 Neurosurgery - Tumours

P-116 to P-118 Neuro-oncology

P-118 to P-140

$\mathrm{P}-141$ to $\mathrm{P}-145$

Education

General Neurology Spinal Cord

obliteration of the AVM will result in dramatic neurological impovement.

We present three patients in whom the diagnosis of spinal dural AVM was seriously delayed (4, 5, and 12 months from onset of symptoms), and the reasons that the correct diagnosis was overlooked. The features of myelography, myelo/CT and MR imaging that should suggest the diagnosis of dural AVM and mandate spinal angiography are highlighted. In 17 patients with dural AVM's, MRI showed T-2 hyperintensity within the conus and lower cord in $16(94 \%)$, swelling of the conus in 11 $(65 \%)$, and a plethora of extramedullary flow-voids in $14(82 \%)$.

A high level of awareness of spinal dural AVM's should prompt the neurologist/neurosurgeon/radiologist to "look high", and search for MR abnormalities remote from the clinical level.

\section{A-2}

Management of Atlantoaxial Instability with Transarticular Screw Fixation

Michael G. Fehlings (Toronto, Ontario)

Although several methods of surgically managing atlantoaxial instability have been described including cerclage wiring or interlaminar clamps, each of these methods has the disadvantage of not controlling rotational movement of the atlantoaxial 
facet joint, having a nonunion rate up to $25 \%$ or requiring postoperative halo immobilization. Recently, posterior transarticular screw fixaton of $\mathrm{Cl}-\mathrm{C} 2$ has been proposed as a superior alternative to conventional fusion techniques. In the present study, the author reports on the results of transarticular $\mathrm{Cl}-\mathrm{C} 2$ fixation in a consecutive series of 15 patients (age $15-81 ; 11 \mathrm{M}, 4 \mathrm{~F}$ ), followed prospectively with a mean followup of $17 \mathrm{mo}$. (range 628 mo.).

The indications for surgery included unstable Type II odontoid fracture $(n=9)$; disrupted transverse atlantal ligament $(n=2)$; rheumatoid atlantoaxial subluxation $(\mathrm{n}=3)$; os odontoideum $(n=1)$; C1-C2 facet joint arthritis with intractable pain $(n=1)$. Four patients had a previous failed Gallie fusion done elsewhere. Custom $3.5 \mathrm{~mm}$ self-tapping transarticular screws were placed under fluoroscopic guidance using instruments designed by the author. The instrumentation was supplemented with a posterior Gallie fusion. Postoperative immobization was in a hard collar for 12 weeks.

There were no vascular or neurological complications. All patients achieved solid union as demonstrated by stability in flexion-extension and bridging trabecular bone. a postoperative $\mathrm{CT}$ scan with reconstructions was performed in all cases to verify correct placement of the transarticular screws. There were no cases of screw malposition.

The author concludes that in experienced hands C1-C2 transarticular screw fixation is a safe, effective technique for managing atlantoaxial instability.

\section{A-3}

Anterior Thoraco-lumbar Spinal Decompression and Antero-lateral Stabilization with the Kaneda System Preliminary Experience

C.B. Agbi, S. Chakravarthi, A.G.E. North, R.R. Anderson, M. Coughlin and R. LEMMON (Windsor, Ontario)

It is now generally accepted that an anterior approach is the optimal method for dealing with anteriorly placed spinal canal compressive lesions. Internal stabilization is almost always necessary following such decompression. Traditional posterior stabilization employing compressive distraction rods suffer from the disadvantages of requiring a separate incision as well as often resulting in constructs that are mechanically unsound. For this reason, recent efforts have shifted to anterior stabilization techniques.

We present an initial experience with anterior decompression followed by anterolateral stabilization using the Kaneda System. Sixteen patients are included in this study. The cause of the compression was metastatic tumor ( 7 patients), traumatic fractures (5 patients), thoracic discs (2 patients), Pott's Disease (1 patient), and primary giant cell tumor ( 1 patient).

There were no operative deaths. One patient with metastatic disease deteriorated neurologically following surgery. In all the others, the neurological condition either improved or stabilized. In all patients presenting with pathological kyphotic deformity it was possible to correct the kyphosis. all patients were mobilized on the first post-operative day and active rehabilitation was commenced at once.

\section{A-4}

\section{Impact of Urodynamic Evaluations on the Surgical Management of Spinal Cord Tethering}

Olivier Vernet, Jean-Pierre Farmer, AnNe-Marie Houle and José LUIS MONTES (Lausanne, Switzerland; Montreal, Quebec)

To determine the usefulness of urodynamic studies in the management of children with suspected tethered cord, we retrospectively reviewed case reports of 21 patients evaluated both pre- and post-spinal cord untethering surgery with this diagnostic adjunct. All patients were investigated with MRI or myelogram-CT. Five patients underwent primary cord untethering whereas 16 patients with prior myelomeningocele closure underwent secondary untethering. The primary surgical indication was neurological ( 9 cases), urological (9 cases), or orthopedic (3 cases) deterioration. Urodynamic investigations were abnormal or aggravated in 15 cases and normal in 6 cases. All patients were operated with microsurgical technique and intraoperative nerve root stimulations. At a mean follow-up of 26 months, 7 out of 9 patients with neurological deterioration were improved, 2 stabilized. Six out of 9 patients with urodynamic aggravation improved, 2 stabilized and one worsened. Two out of 3 patients with orthopedic presentation improved and one stabilized. Of the 21 patients studied urodynamically, 11 improved, 9 stabilized and one worsened postoperatively. We conclude that urodynamic studies are useful both diagnostically and in the follow-up of patients with tethered cord, that urodynamic disturbances often precede clinical deterioration and that untethering of the cord influences favorably the urodynamic status of these patients.

\section{A-5}

\section{What Use is Chronic Stimulation for Pain?}

\section{R.R. Tasker, Andrew Parrent and Osvaldo Vilela Filho} (Toronto, London, Ontario; Goiania, Brazil)

3 decades of chronic stimulation has given conflicting results. For neuropathic pain using 3 modalities, half our patients reported relief during trial stimulation but $\%$ with $>$ $50 \%$ relief $>1$ year depended on diagnosis (Table). Postherpetic and amputation pain were particulary intractable. DCS was unsuccessful for central pain. Otherwise, if adquate DCS failed, so did DBS. Only parethesiae-producing (capsule, thalamus, lemniscus), not pVG, stimulation affected steady neuropathic pain. $54 \%$ of cases of DCS that failed after internalization did so in the first 3 months. Although VS and DS sometimes relieved allodynia from peripheral lesions, DBS was usually painful in stroke pain with allodynia.

\begin{tabular}{lccccccc}
\hline & \multicolumn{2}{c}{ VSTIM } & \multicolumn{2}{c}{ DCS } & \multicolumn{2}{c}{ DBS } \\
\hline Lesion & $\mathbf{N}$ & $\begin{array}{c}\% \\
\text { relief }\end{array}$ & $\mathbf{N}$ & $\begin{array}{c}\% \\
\text { relief }\end{array}$ & $\mathbf{N}$ & $\begin{array}{c}\% \\
\text { relief }\end{array}$ \\
\hline Peripheral & 24 & 33 & 102 & 39 & 27 & 37 \\
\hline Cord & - & - & 31 & 0 & 16 & 35 \\
\hline Brain & 6 & 83 & 12 & 8 & 17 & 32 \\
\hline
\end{tabular}




\section{A-6}

Clinico-pathological Correlations in Cushing's Disease: a Longitudinal Study of Surgical Failure and Recurrence Rates

amin B. Kassam, H. Grossman, H. Smyth, K. Kovacs, E. HoRVATH and S. EzZAT (Toronto, Ottawa, Ontario)

Despite significant refinements over the last three decades in both diagnostic and microsurgical techniques, the failure and recurrence rates in patients with surgically treated Cushing's disease remains as high as $15-20 \%$. Further to study these problems, we retrospectively reviewed 102 consecutive cases of biochemically and clinically documented Cushing's disease treated surgically at the Wellesley Hospital from 1977 through 1994.

The disease primarily affects females (5.8:1), and is detected at a mean age of $35.3 \mathrm{yr}$. MRI proved to be the most valuable imaging method, correctly lateralizing a lesion within the pituitary gland in $87 \%$ of 48 cases. Simultaneous bilateral inferior petrosal sinus sampling was not as reliable. While intrasellar localization of an ACTH source was $100 \%$, lateralizing gradients matched surgical findings in only $56 \%$ of 16 cases.

We report a spectrum of histological and pathological findings including: "normal gland only" $(20 \%)$; corticotroph cell hyperplasia (9\%); microadenoma (67\%); and macroadenomas $(7 \%)$. The overall failure rate was $19.6 \%$. The recurrence rate after sustained remission was $16.6 \%$, with delayed recurrences arising as late as 14 and 16 years postoperatively. The immunohistochemical demonstration of alpha subunit in surgically resected tissue appears to be a predictor of likely failure ( $\mathrm{p}=$ $0.038)$ and of recurrence ( $p=0.00024)$. Alpha subunit is not a feature of normal corticotrophs, and, while its exact significance in this context is not clear, its presence may prove an indication for closer monitoring of patients in remission. Further study of this finding may illuminate the complex relationship between the pathogenesis of Cushing's disease and the heterogeneous spectrum of histopathologic findings with which it is associated.

\section{A-7}

MRI Follow-up after Transsphenoidal Surgery: Does it Make a Difference?

\section{J.F.R. Fleming and W. MONTANERA (Toronto, Ontario)}

We report the findings in serial MR scans following transsphenoidal surgery in 100 consecutive patients with pituitary macroadenomas $(>1 \mathrm{~cm})$, with a mean follow-up of 6 years. MR scans were done 3 months postoperatively and annually thereafter. The postoperative pituitary gland may be identified by its shape, size, location and enhancing characteristics but is not always distinguishable from residual tumour.

Of the endocrine-inactive macroadenomas (which tended to be very large), the initial MRI suggested no residual tumour in $35 \%$ of patients, some residual tumour in $55 \%$, and a large amount of residual tumour requiring an early second operation in $10 \%$. There was MRI evidence of tumour recurrence in $6 \%$ of those patients whose initial postoperative MRI showed no residual tumour. Of those with some residual postoperative tumour, there was no subsequent enlargement during our follow-up period (range $2-10$ years) in $83 \%$, and progressive enlargement in $17 \%$.

We recommend a baseline MR scan 3 months postoperatively (long enough for post-surgical changes to have stabilized) in all patients, regardless of the surgeon's estimation of totality of removal. A valuable by-product of post-op MRI is feedback to the surgeon, which encourages aggressive tumour removal with the goal of as radical and complete removal as possible. All patients with endocrine-inactive adenomas should have an annual follow-up scan. Management of patients with residual or recurrent tumour is individualized and may include either continuing MRI follow-up, repeat surgery, and/or radiotherapy.

\section{A-8}

Functional Magnetic Resonance Imaging (fMRI) in Neurosurgical Patients

E.T. Kiriakopoulos, D.J. Mikulis, M.L. WoOd, M. BERnSTEIN, C. Wallace, J. Drake, H. Hoffman, A. Lozano and S. Chung (Hamilton, Toronto, Ontario)

Purpose: To investigate the utility of localizing regional brain activity with fMRI in adult and pediatric neurosurgical patients.

Methods: fMRI studies in 17 patients with focal space occupying lesions were reviewed. Patients underwent testing to evaluate either motor, sensory, language or visual cortical activity. Images were obtained on a conventional $1.5 \mathrm{~T}$ instrument using the following parameters: TR 68 , TW 40 , matrix $256 \times 128$, field of view $30 \times 22 \mathrm{~cm}$, flip angle 45 degrees and $4 \mathrm{~mm}$ slice thickness. Activated regions were identified with a pixel-bypixel t-test analysis comparing the mean signal intensities of the resting and task images.

Results: In 15 of 17 patients the relationship between functional cortex and lesions was successfully demonstrated, based on classical neuroanatomy of the brain, with fMRI. Mappings were confirmed by electrocortical stimulation in 5 patients.

Conclusion: fMRI can demonstrate, using simple motor language paradigms, the relationship of regional brain activity to focal lesions in adult and pediatric patient populations. Presurgical mapping of vital brain regions is an appealing clinical application of fMRI. Intra-operative electrocortical stimulation has confirmed the ability of fMRI to accurately display the relationship between sensorimotor and language areas and resectable lesions.

\section{A-9}

Hearing Preservation in Intracanalicular Acoustic Neuroma Removal by the Retrosigmoid Approach

\section{D.W. Rowed and J.M. NEDZELSKi (North York, Ontario)}

In 472 consecutive acoustic neuroma excisions, hearing preservation by a retrosigmoid approach was attempted in 100 cases. In 79 patients intraoperative auditory function was monitored by means of cochlear compound action potentials (CAPs) and brainstem auditory evoked potentials (BAEPs). Twenty hearing preservation attempts $(20 \%)$ were in cases of predominantly intracanalicular tumours which extended $\leq 4 \mathrm{~mm}$ medial to the porus acusticus. In the intracanalicular group, serviceable hearing [speech reception threshold (SRT) $\leq 50 \mathrm{~dB}$, speech discrimination score (SDS) $\geq 60 \%$ ] was preserved in $10 / 20$ patients (50\%), compared with $19 / 59$ (32\%) of monitored patients with tumours $5-20 \mathrm{~mm}$ in extracanalicular diameter. (Overall success rate for serviceable hearing preservation was $38 \%$ ). Thus there appears to be a trend to improve hearing preservation in intracanalicular tumours which is not statistically significant $(P=$ 0.18 ). Results in the present series do not differ significantly 
from recent reports which suggest that higher success rates for hearing preservation in intracanalicular acoustic neuromas may be achieved via the middle cranial fossa approach. The retrosig$\mathrm{moid} /$ suboccipital approach is safe, familiar to all neurosurgeons and achieves a relatively high rate of hearing preservation. Features of surgical technique which we believe to be important will be discussed in detail.

\section{A-10}

Intraoperative Somatosensory Evoked Potential (SSEP) Identification of Sensorimotor Cortex in Intracranial Tumour Resection: Clinical Experience

\section{D.W. Rowed and D.A. Houlden (North York, Ontario)}

Phase reversal of the cortically generated median N20/P30 SSEP complex across the central sulcus was used to identify sensorimotor cortex intraoperatively in 40 patients under general anesthesia. All patients had intracranial neoplasms invading or compressing the frontal or parietal lobes (18 metastatic carcinomas, 16 gliomas and 6 meningiomas). SSEP recordings were obtained from a 4 lead strip electrode placed directly on the brain. We report our experience correlated with visual identification based on anatomical landmarks, and with preoperative MR and CT imaging.

In 36 patients (90\%) SSEPs showed consistent phase reversal. In 2 patients (5\%) intraoperative SSEPs were absent and in 2 SSEPs were present but without consistent phase reversal. In 7 cases $(18 \%)$ electrophysiological localization of sensorimotor cortex differed from visual identification at operation. In 5 this was due to displacement by tumour. In 2 cases SSEP localization of sensorimotor cortex was successful despite out inability to visually localize the sonsorimotor cortex due to distortion of surface anatomy by tumour. In 4 patients $(10 \%)$ tumour resection was modified because of SSEP findings.

SSEP is a simple, inexpensive and reliable method of identifying sensorimotor cortex under general anesthesia and is not affected by cortical displacement at operation. This technique appears to be underutilized in the practice of neurosurgery.

\section{B. NEUROLOGY - GENERAL ( $B-1$ to $B$-4) (Thursday, June 22, 14:00 - 15:15)}

\section{B-1}

\section{Post-concussion Syndrome: Clinical - MR Brain Relationships}

Gordon Cheung, Sandra Black, Michael Schwartz, Perry COOPER and RICHARD FARB (Toronto, Ontario)

Purpose: To evaluate the relationship between clinical severity measures and MR parencymal brain abnormalities in post-concussion syndrome.

Methods: Positive MR brain scans were retrospectively reviewed in 12 patients with post-concussion syndrome ( 8 males and 4 females, ages 17-47) consecutively referred for medicallegal or compensation assessment. The relationship of lesion abnormalities to duration of loss of consciousness (LOC), Glasgow Comma Scale (GCS), retro and anterograde amnesia and neuropsychological testing was examined.

Results: Only 2/12 patients had parenchymal lesions on CT. On MRI, 9 patients had punctate old hemorrhages ( $\mathrm{N}=3$ to $>20$ ) at the grey-white junction, corpus callosum or midbrain, best seen on gradient-echo images. Two patients had cortical contusions and one had multiple white matter lesions on spin-echo. No clear relationship emerged between clinical severity measures and the number of MR lesions. In one severe head injury with poor outcome, only three hemorrhages were seen; other patients with more numerous hemorrhages had relatively mild neuropsychological sequalae.

Conclusions: If confirmed in a larger sample, our finding that the number of MR lesions did not clearly correlate with clinical severity measures will be important in the interpretation of medical-legal and compensation cases.

\section{B-2}

Pathophysiology of HTLV-I Associated Myelopathy: Increased Spontaneous DNA Synthesis, Response to HTLV-I Envelope Protein, and Inhibition by Myelin Basic Protein

Joel J.-F. Oger, G. Dekaban, T. Lu, T. Aziz, L. Elder and J. Arp (Vancouver, British Columbia; London, Ontario)

We have recently reported that HTLV-I Associated Myelopathy (HAM)/Tropical Spastic Paraparesis (TSP) is endemic among Coastal Natives in B.C. To explore the pathogenesis of this demyelinating disease, we have tested cell mediated immunity (CMI) in Native HAM/TSP patients $(n=5)$, Native seropositive carriers $(n=7)$, Caucasian M.S. patients with myelopathy $(n=8)$ and healthy controls, both Native $(n=5)$ and Caucasian $(n=12)$. CMI was evaluated by DNA synthesis (tritiated thymidine uptake) in cultured ( 3 and 6 days) blood lymphocytes stimulated with HTLV-I viral envelope protein (ENV-IB, 0.05 and $0.10 \mu \mathrm{g} / \mathrm{ml}$ ), guinea-pig myelin basic protein (MBP, gift of Dr. J. Whitaker), or pokeweed mitogen (GIBCO: 1/300 dilution).

We verify that spontaneous DNA synthesis is significantly higher in HAM/TSP $(38,974 \pm 8,612$ mean \pm SEM $)$ than in carriers $(21,620 \pm 5,850)$, M.S. $(6,031 \pm 1,209)$, or healthy controls $(16,779 \pm 3,297) .3$ of 5 HAM/TSP but only 1 of 7 carriers, 2 of 8 M.S., and 2 of 15 healthy controls showed a substantial increase $(10,000 \mathrm{cpm}>$ spontaneous) in proliferative response to ENV-IB. Spontaneous proliferation was reduced in HAM/TSP and carriers when MBP was added at $1 \mu \mathrm{g} / \mathrm{ml}$, a concentration determined not to reduce PWM induced proliferation in helathy controls.

These data suggest that in HAM/TSP lymphocytes are sensitized to ENV-IB and MBP turns down spontaneous DNA synthesis.

\section{B-3}

Psychometric Prediction of Progression to Dementia in Subjects with Aging Associated Cognitive Decline (AACD)

R. McKelvey, H. Chertkow, L. Babins, N. Kelner and H. BERGMAN (Montreal, Quebec)

Non-demented elderly subjects with mild cognitive decline have been demonstrated to be at high risk for progression to dementia. Several groups of investigators have identified neuropsychological investigations that are sensitive to mild cognitive decline and may predict progression. We have administered a battery of neuropsychological tests reported from three such labs to a group of elderly subjects who fit the criteria for AACD by Levy et al. (1994). We have followed thirty such subjects for an average of 14 months, during which eight subjects have progressed to dementia. The Enhanced Cued Recall task reported by Grober et al. (1988) demonstrated a good correlation with subsequent progression to dementia (Kappa .56). Abnormalities on a group of standard tests used by Morris et al. (1991), and on another group of 
tests constructed by Flicker, Ferris and Reisberg (1991) showed only mild correlation with progression to dementia (Kappa $<.4$ ). Clinical impressions of the neurologists and psychologists were recorded separately, and each showed poor correlation with subsequent decline (Kappa <.3). We conclude that the best predictor of progression to dementia in AACD subjects is the demonstration of objective memory loss as measured by the enhanced cued recall test.

\section{B-4}

Kennedy's Disease: a Rare But Important Clinical Subtype of Motor Neuron Disease

\section{T.J. Benstead and R.A. Purdy (Halifax, Nova Scotia)}

$\mathrm{X}$-linked bulbospinal muscular atrophy (Kennedy's Disease) can be mistaken for acquired motor neuron disease (MND), if the family history is not obvious. We evaluated two patients initially felt to have acquired MND, who were eventually diagnosed with Kennedy's Disease. Patient 1 age 68, had a 5 year history of progressive weakness. He had speech and swallowing difficulties and extremity weakness. Two maternal uncles had late onset progressive weakness. Patient 2 age 57, had a 3 year history of progressive dysphagia and extremity weakness. No family members were known to have neuromuscular disease. Both patients had prominent facial fasciculations, normal sensation, no upper motor neuron features, diffuse EMG denervation and mild CK elevation. Kennedy's Disease was confirmed by demonstrating expansion of the CAG repeats in the 5' region of the androgen receptor gene. These cases highlight the features of Kennedy's Disease, which can easily be mistaken for acquired MND, lower motor neuron type. The relevant family history may be absent. Prominent facial fasciculations, other features of early bulbar disease and elevated CK may raise clinical suspicion of the disorder. Correct diagnosis is important for appropriate counseling regarding prognosis and genetics.

\section{CEREBROVASCULAR DISEASE ( $C-1$ to $C-6$ )}

(Thursday, June 22, 15:30 - 17:00)

\section{C-1}

Cerebrospinal Fluid D-Dimer Assay: a More Sensitive and Specific Test for the Diagnosis of Subarachnoid Haemorrhage?

\section{C.R. HoNEY (Vancouver, British Columbia)}

This study compares the sensitivity and specificity of a Ddimer assay with red blood cell (RBC) counts in the cerebrospinal fluid (CSF) of patients suspected of having had a subarachnoid haemorrhage (SAH).

CSF was examined from 38 consecutive patients having had either i) a lumbar puncture for suspected SAH, of ii) a lumbar drain prior to craniotomy for aneurysmal bleed. Four samples of CSF were examined for a) RBC count in tube 1 , b) percentage change of RBC from tube 1 to tube 4 , c) xanthochromia, d) DDimer concentration (ORTHOTM Dimertest, Ortho Diagnostics System). A positive $\mathrm{RBC}$ test was defined as $\mathrm{RBC}_{\text {tube } 1}>1000 \mathrm{x}$ $10^{6} / \mathrm{L}$ and $<50 \%$ drop from $\mathrm{RBC}_{\text {tube }}$ to $\mathrm{RBC}_{\text {tube } 4}$. A positive $\mathrm{D}$ Dimer Assay was defined as $\geq 200 \mathrm{ng} / \mathrm{ml} \mathrm{D}$ dimer. These results were compared against the gold standard of CT scan and cerebral angiogram.
The D dimer assay sensitivity $(0.80)$ and specificity $(0.87)$ fell surprisingly below that for RBC count sensitivity $(0.87)$ and specificity (1.0). The pretest prevalence of SAH in our patient population was 0.39 . Using RBC counts, this resulted in a postpositive test likelihood PTL $(+)=1.0$ and a post-negative test likelihood PTL $(-)=0.077$.

The implications of these tests on the diagnosis of SAH in different community settings is discussed. We conclude that CSF $\mathrm{RBC}$ cell counts is a very sensitive and highly specific test for the diagnosis of SAH.

\section{C-2}

\section{Carotid Stenosis in Lacunar and Cortical TIA's}

\section{B.J. STEWART and A. SHUAIB (Saskatoon, Saskatchewan)}

Previously, it has been reported that cortical TIA's are associated with a significantly greater degree of ipsilateral carotic stenosis than are lacunar TIA's. We retrospectively analyzed 122 patients with TIA's, 30 of which underwent cerebral angiography. Symptoms were associated with $50 \%$ or greater ipsilateral carotid stenosis in 5 out of 16 patients (31.3\%) with presumed lacunar TIA's and in 5 out of 14 patients $(35.7 \%)$ with presumed cortical TIA's. In contrast to earlier reports, our findings indicate that there is no significant difference in the degree of ipsilateral carotid stenosis in patients suffering lacunar TIA's versus those suffering cortical TIA's. This supports the idea that lucunar TIA's can frequently arise from emboli originating in the ipsilateral carotid artery as well as from lipohyalinosis. Our data suggests that all patients experiencing a presumed lacunar TIA should have a complete workup which includes carotid ultrasound and, if necessary, cerebral angiography.

\section{C-3}

Lesion Correlates of Persisting Hemi-spatial Neglect in a Consecutive Stroke Population

\section{S.E. BLACK and P. EBERT (North York, Scarborough, Ontario)}

Objective: To investigate lesion parameters in persisting hemispatial neglect. Background: There are few studies of neglect recovery and even fewer with lesion correlations. Method: In a longitudinal study of 453 consecutive strokes, 294 patients underwent standardized neglect testing within 14 days of onset. Neglect was classified as severe (score 40-100) or mild (6-39). If neglect was present patients were recalled at 1 month, 3 months, and 1 year. Results: Neglect was initially present in $31 \%$ of left-hemisphere-damaged (LHD) and $54 \%$ of right-hemisphere-damaged (RHD) subjects. It was severe in $21 \%$ of RHD and $0.8 \%$ of LHD subjects. Most recovery occurred by 3 months. Of the 19 RHD subjects with initial severe neglect who were testable at I year, $32 \%$ still demonstrated severe and $32 \%$ demonstrated mild neglect. Lesion volumes were not significantly different in RHD patients with and without recovery. Overlapping of lesion localizations identified common damage to the temporo-occipital (TO) junction in persisting neglect. Those who recovered had more anterior lesions, sparing this area. Conclusions: Neglect recovers in the majority of patients by 3 months. Damage to white matter pathways, deep to temporo-occipital cortex appears to impede restoration of visuospatial attention. 
C-4

\section{Quality of Life After Stroke}

\section{P.J. Clarke, J.M. Lawrence and S.E. Black (Toronto, Ontario)}

While physical disability is often perceived to be the main determinant of quality of life following stroke, we found that there are other factors influencing the life satisfaction of stroke survivors.

In a prospective cohort study of 439 consecutive hemispheric strokes we examined the factors associated with quality of life at three months and one year post stroke in 131 surviving patients who could be meaningfully assessed. Greater independence in mobility and cognition, as measured by the Functional Independence Measure (FIM), were statistically significant independent predictors of a better quality of life, as measured by the Reintegration to Normal Living Index (RINL), explaining $56 \%$ and $51 \%$ of the variance $\left(\mathrm{R}^{2}\right)$ in RINL at three months and one year, respectively. The large multiple correlation coefficients of these two linear models suggests that over half of the variance in the quality of life of stroke survivors is explained by physical and cognitive disability. However, not all individuals who remained functionally dependent reported a poor quality of life. For instance, physically disabled individuals reported an improvement in their quality of life from three months to one year even though there was no improvement in disability.

While disability may explain over half of the variability in quality of life following stroke, our results suggest that there are also other important factors which account for an individual's satisfaction in social roles. These factors need to be identified so that health care professionals will have more ability to assist in the restoration of optimal quality of life following stroke.

\section{C-5}

\section{Factors Affecting Survival in Life-threatening Stroke}

Natalia A. Alexandrova, Patrick M. Pullicino, Liliana T. Smurawska, Andrei V. Alexandrov and John W. Norris (Toronto, Ontario; Buffalo, New York)

Background: Currently available data lacks precision in quantifying life-threatening brain damage on CT, and the disease-specific criteria for futility of life-sustaining interventions in acute stroke remain uncertain.

Results: Consecutive patients who died within 3 months following acute stroke $(n=111)$ were studied. Control group comprised of patients with acute severe stroke who were discharged from the hospital $(n=35$, Canadian Neurological Scale scores $<$ 5.0 on admission). There were 68 patients who died within the first two weeks after stroke (mean time $5 \pm 4$ days) and 43 died later (76 \pm 91 days). CT-volume of the lesion was greater in the early death group $(248 \pm 233 \mathrm{ml})$ compared to $140 \pm 191 \mathrm{ml}$ in the delayed death group and in the control group $(129 \pm 132 \mathrm{ml}, \mathrm{p}<$ 0.01 ). Time of death following stroke was inversely related to the lesion volume, septum pellucidum (SD) and pineal gland displacement (PD). All patients with $S D>12 \mathrm{~mm}$ and $\mathrm{PD}>5 \mathrm{~mm}$ died early irrespective of treatment. Surgical decompression and ventilator-assisted breathing were used in $12 \%$ of patients with fatal stroke and in $26 \%$ in the control group (ns).

Conclusions: Measurements of lesion volume and midline shift or herniation are useful in predicting inevitable fatal outcome in acute stroke and may be used for limiting the aggressiveness of treatment.
C-6

NMDA Antagonists: a Reappraisal - Protection Against Transient Forebrain Ischemia in Rodents by the Competitive NMDA Antagonist Remacemide

\author{
H.J. Lesiuk, K. BARnes, M.J. Todd and K. Hewitt (Ottawa, \\ Ontario)
}

Efficacy of NMDA antagonists as protectants against cerebral ischemia, in vivo, remains controversial. Much literature centres on the non-competitive NMDA antagosits typified by MK-801 (Dizocilpine Maleate); some have suggested "protection" by these agents is due to effects on temperature or cerebral blood flow rather than NMDA antagonism. To further probe this issue, we evaluated the competitive NMDA antagonist, Remacemide, in a rodent model of transient forebrain ischemia.

Twenty-three male Sprague-Dawley rats $(250-300 \mathrm{~g})$ maintained normothermic under general endotracheal anaesthesia and mechanical ventilatory support, were subjected to transient (10 min) bilateral carotid occlusion plus systemic hypotension (50 torr). Post ischemia, they received either Remacemide $(20 \mathrm{mg} / \mathrm{kg}$ ip in $1.0 \mathrm{ml}$ saline upon reperfusion, followed by $10 \mathrm{mg} / \mathrm{kg}$ ip 4 and 24 hours later) or equivalent volumes of saline. Meticulous temperature regulation was maintained post-ischemically. After 7 days animals were perfusion fixed and standard coronal brain $\mathrm{H}$ \& E sections prepared. Normal and ischemically damaged hippocampal CAl neurons were counted and the percentage ratios of ischemic to total neurons calculated.

Remacemide treated animals $(n=15)$ sustained $(58.1 \pm 12.2 \%$ damage, mean \pm S.D.), significantly less ( $p<0.02$, Mann-Whitney $U)$ than control animals $(n=8,72.8 \pm 9.7 \%$ damage $)$. We conclude Remacemide has significant neuroprotective effects not dependant on induced hypothermia.

D. CLINICAL NEUROPHYSIOLOGY ( $D-l$ to $D-5)$ (Thursday, June 22, 14:00 - 15:15)

\section{D.1}

\section{Lambert-Eaton Myasthenic Syndrome Presenting as Ventilatory Failure}

D.J. Stewart, R. Chen, M.W. Nicolle and C.F. Bolton (London, Ontario)

Lambert-Eaton myasthenic syndrome (LEMS) is a rare presynaptic neuromuscular transmission disorder, characteristically presenting with the triad of proximal muscle weakness, hyporeflexia and constipation. Ventilatory failure is rarely a presenting feature.

We report on two cases of LEMS presenting with ventilatory failure. Antibodies against the presynaptic voltage-gated calcium channel were measured in Case 1 and were markedly elevated. Case 1 was associated with squamous cell carcinoma in situ and Case 2 was associated with small cell carcinoma of the lung.

Electrophysiologic studies in both cases showed characteristic low amplitude compound muscle action potentials (CMAPs) and a $50-100 \%$ incremental response to fast $(40 \mathrm{~Hz})$ repetitive nerve stimulation. Phrenic nerve conduction studies showed either absent (Case 1) or reduced (Case 2) diaphragmatic CMAP. Needle EMG of the diaphragm showed markedly decreased recruitment (Case 1) or no voluntary units recruited (Case 2).

Case 1 was ventilatory-dependent for six weeks, Case 2 for only four weeks. Both cases were weaned from ventilation following treatment with plasma exchange, 3,4-diaminopyridine, prednisone and pyridostigamine. 
LEMS should be considered in the differential diagnosis of patients presenting with unexplained ventilatory failure, expecially those with reduced CMAPs. Electrophysiological studies are useful to demonstrate severe involovement of the respiratory system in neuromuscular transmission disorders.

\section{D-2}

\section{Radial Neuropathy: Rare Mononeuropathy With a Poor} Outcome?

\section{MARTIN VeILLEUX (Montreal Quebec)}

Radial motor and/or sensory neuropathy is the least common mononeuropathy in the upper extremity. From January 1992 to December 1994, 6386 patients were evaluated in the EMG Laboratory, of whom 19 had symptoms and signs consistent with a radial neuropathy. There were 12 women and 7 men with a mean age of 45.5 years (range: $20-88$ ). Clinical findings suggested a mixed motor and sensory radial neuropathy in 8 patients, a radial motor neuropathy in 8 , and a radial sensory neuropathy in 3 . Eight patients had a moderate to severe weakness of the wrist and/or finger extensor muscles. Causes for radial mononeuropathy included radial nerve compression proximal to the elbow in 5 patients, fracture of humerus in 4, resistant tennis elbow or following lateral epicondylectomy in 3 , laceration in the distal arm in 1 , and unknown in 6 . Nerve conduction studies and concentric needle examination of radial and non-radial innervated muscles revealed findings consistent with a radial neuropathy in all patients. Mean length of follow-up was 12.3 months (range: $2-42$ ). Overall, recovery was poor except in patients with radial nerve compression proximal to the elbow (Saturday night palsy).

\section{D-3}

\section{Functional MRI Evaluation of Visual Evoked Responses}

W.J. Logan, K.K. Kwong, R.B.H. Tootell, V.S. Caviness and B.R. Rosen (Charlestown, Massachusetts)

Several visual processing streams and cortical centres have been identified in the primate brain which are selectively activated by different visual stimuli. Functional imaging studies in the human have also indicated that there are different cortical areas for vision (e.g., VI, V4, MT/V5; Tootel et al., Soc. Neurosci. Abst. $350.1-350.5,1994)$. There is evidence that clinical visual evoked potentials (VEPs) produced by different stimuli (e.g., flash, pattern) have different morphology, latency and topography in the same individual. To determine if this is due to activation of different cortical areas, we used a funtional magnetic resonance imaging technique (fMRI) which has been shown to detect activation produced by visual stimulation.

We studied adult volunteers using a $1.5 \mathrm{~T}$ GE MR imager equipped with echoplanar technology for fast imaging. A headcoil was used for effective whole brain scanning. Visual stimulations with the different VEP stimuli used clinically were performed in the same subject. During imaging these stimuli were presented at $1-4 \mathrm{~Hz}$, and the areas demonstrating significant change in signal intensity with activation were compared for the different stimuli.

Each type of visual stimulation produced prominent occipital cortex activation of a region in the occipital cortex, but in contrast to pattern, flash activation tended to be more circumscribed, medial and anterior. It appears that flash more selectively activates V1 whereas pattern may produce more extrastriate activation in areas such as MT/V5. Thus, while these VEP stimuli each activate visual cortex, different cortical areas are involved, and this may help explain the electrophysiological differences that have been described.

\section{D-4}

\section{Prediction of Outcome in Anoxic Coma With EEG and Evoked Potential}

\section{R. CHEN, C.F. Bolton and G.B. Young (London, Ontario)}

Neurological prognosis is often the most important question in survivors of cardiac arrest. Reliable prediction of outcome can facilitate subsequent management decisions. We studied 22 patients (age 17-80, mean 58) who remained in coma 24 hours after cardiac arrest or severe hypotension. In all patients neurological examination, EEG and median nerve somatosensory evoked potential (SEP) studies were performed within four days of coma. Three survived with good outcome and 19 died. All three survivors had normal N20 component of SEP, one had benign EEG and 2 had malignant EEG. Among the 19 patients who died, 4 had normal, 5 had low amplitude and 10 had absent SEP. The results of EEG in this group were: 1 benign, 3 uncertain and 15 malignant. Two patients with malignant EEG and normal SEP survived with good outcome. Three patients whose SEP amplitude declined in follow up studies all died. SEP may improved outcome prediction based on EEG and clinical features. It can reinforce the impression of fatal or poor outcome and is useful in following disease progression. Some patients with normal SEP but poor prognosis based on EEG and clinical criteria may survive with good outcome.

\section{D-5}

Sensory Evoked Potentials Recorded From the Posterior Fossa: Analysis of a Non-Cephalic Reference

John R. Hurlbert and Michael G. Fehlings (Toronto, Ontario)

Evoked potentials recorded from over the cerebellar hemispheres (CEPs), arising from lower limb stimulation, have been shown to be carried in the ventral funiculus in animal studies. A similar evoked potential has been reported in human patients, and has been hypothesized to provide a method for evaluation of the electrophysiological integrity of the ventral spinal cord. The purpose of this experiment was to evaluate the contribution of active and reference recording sites to these evoked responses, using cephalic (Fpz) and non-cephalic (shoulder) references.

Although the diminished signal-to-noise ratio made recordings difficult to obtain, we were able to record responses from over the cerebellum (Occ) in all patients studied $(n=10)$. The initial negative Occ-Fpz activity (N33) was seen in the non-cephalic (Occshoulder) recordings. The subsequent positive component (P40) was also present when referenced to the shoulder. However, a negative peak of slightly later latency, seen in Fpz-shoulder recordings, contributed a variable amount to the amplitude of P40.

These results demonstrate that in humans, evoked potentials recorded over the cerebellum from lower limb stimulation, arise from activity seen by both the active and reference electrodes. The Occ-Fpz response may prove useful as an adjunct to somatosensory evoked potentials for intra-operative spinal cord monitoring.

\section{E. MOVEMENT DISORDERS ( $E-l$ to $E-6$ )}

(Thursday, June 22, 15:30-17:00)

\section{E-1}

\section{Lewy Body Parkinson's Disease in a Large Kindred}

A.H. Rajput, M. Fenton, D. George, Alex Rajput and B. KANIGAN (Saskatoon, Saskatchewan; Iowa City, lowa)

Large kindreds of idiopathic Parkinson's disease (PD) have been reported but most cases are clinically atypical-young onset, 
no tremor, prominent dementia and short survival. We report a large kindred of 4 generations with 9 definite and 1 possible case. In generation I, the patriarch developed parkinsonian symptoms in his 70 s but no medical documentation exists. In generation II, 5 of 8 siblings (7M, IF) developed PD - all evaluated personally (AHR). Mean onset age was 72.6 years. One brother who died at age 88 after 12 years of PD had Lewy body pathology. The 5 affected brothers shared the same family residence and manifested an inverse relation between time in family residence and onset age. In generation III, three cousins (with PD fathers) developed PD at mean 52.3 (49-59) years. In generation IV, a 23-year-old male displayed early PD. All affected members spent childhood on farms in the same general area. The transmission in this pedigree conforms to a multifactorial-threshold (MFT) model and to an autosomal dominant - low penetrance model. The onset age is younger in successive generations. The anticipation phenomenon suggests greater genetic loading. The reasons for only males having PD are unclear. Similar activities by same-sex siblings and/or hormonal protection are among the possibilities.

Details of this pedigree and significance to the etiology of PD will be presented.

\section{E-2}

\section{Levodopa Complications in Idiopathic Parkinson's Disease}

A.H. Rajput, D. George, Alex Rajput and Mark Fenton (Saskatoon, Saskatchewan; Iowa City, Iowa)

Long term levodopa (LD) therapy in parkinsonism (PS) frequently results in dyskinesias, wearing off and on-off. The pathophysiology of these complicaitons, however, remains unknown. Based on clinical observations it is believed that an early onset age predisposes to dyskinesias and the late onset to motor response fluctuations. Most previous studies have included all variants of Parkinson syndrome and are of short duration followup.

We conducted clinical and pathological studies in 28 Lewy body parkinsonian cases. 25 cases were given adquate trial on $\mathrm{LD}$. The median followup was $6.7(0.25-21.5)$ years. All patients had initial improvement on LD and $36 \%$ maintained some benefit during the last year of life. Combination of all - dyskinesias, wearing off and on-off were observed in $12 \%$ while $40 \%$ cases had none of those side effects. $24 \%$ patients had only dyskinesias and $22 \%$ only the wearing off but no one manifested on-off phenomenon without experiencing dyskinesias and/or wearing off. These side effects did not correlate with the onset age, sex, severity of motor disability at initial assessment, duration of followup or the length of illness before death.

Details of different response profile and possible basis of these LD complications will be presented.

\section{E-3}

Pre-synaptic Nigrostriatal Dysfunction Predates Clinical Symptoms in Carriers of The PPND Gene

A. Kishore, F.J. Vingerhoets, B.J. Snow, F. Arwert, Z.K. Wszolek and D.B. CAlne (Vancouver, British Columbia; Amsterdam, Netherlands; Omaha, Nebraska)

Objective: To determine pre-synaptic nigrostriatal dopaminergic function using FD PET in genetically confirmed carriers of PPND.

Background: Rapidly progressive autosomal dominant parkinsonism and dementia with pallido-ponto-nigral degeneration (PPND) begins in the fifth decade and runs an inexorable, fatal course. FD PET has shown marked reduction in striatal uptake in
PPND. The gene has a $95 \%$ penetrance and carriers may have presymptomatic disease. PET has shown pre-clinical abnormalities in other forms of parkinsonism.

Methods: FD scans were performed on four genetically confirmed (90\% confidence) asymptomatic carriers, eight non-gene carrier relatives, four symptomatic individuals and ten agematched controls. FD scans were analysed using a graphic model to calculate the striatal uptake rate constant. The results were compared using ANOVA.

Results: The FD striatal uptake constant was significantly reduced in gene carriers $(25 \%)$ and symptomatic individuals $(63 \%),(p=<0.01)$. The gene negative relatives had normal values. There was no trend towards an age-related decline in striatal uptake in gene carriers.

Conclusion: Pre-synaptic nigrostriatal dopaminergic function is abnormal in clinically asymptomatic carriers, though less than in symptomatic individuals. The accelerated decline in nigrostriatal function in PPND appears to occur in the fifth decade when the disease manifests.

\section{E-4}

Lesions of the Subthalamic Nucleus Suppress Tardive Dyskinesia-like Movements in the Rat

\section{A.J. StoEsSL (London, Ontario)}

Although tardive dyskinesia (TD) is a common complication of neuroleptic treatment, the mechanisms are poorly understood. Current models of basal ganglia function suggest that the subthalamic nucleus (STN) plays a critical role in the development of dyskinesias and stimulation of D1 dopamine receptors in this region is known to elicit oral movements.

Male SD rats were treated for 24 weeks with fluphenazine decanoate (FLU; $25 \mathrm{mg} / \mathrm{kg}$ i.m.) or its vehicle (VEH; sesame oil 1 $\mathrm{ml} / \mathrm{kg}$ i.m.) q 3 weeks. Bilateral STN lesions were made with quinolinic acid ( $100 \mathrm{nmol} / 1 \mu \mathrm{l}$ per side). Sham lesions were made by infusing the quinolinate vehicle. FLU induced increased vacuous chewing movements in sham lesioned rats (a putative model of TD) which were attenuated to control levels by lesions of the STN. STN lesions resulted in increased locomotion and sniffing in VEH-treated animals, but this was markedly suppressed by FLU. Rearing and grooming were also suppressed by FLU in the lesioned animals.

If neuroleptic-induced VCMs are indeed analogous to TD humans, the present data suggest that TD is associated with increased activity in the STN, in keeping with the model recently proposed by Trugman et al. (1994).

\section{E-5}

Clinical and Experimental Evidence of Persistent Parkinsonism and Dopaminergic Cell Loss in Substantia Nigra Following Neuroleptic Treatment

\section{M.F. MAZUREK and P.I. RosebuSH (Hamilton, Ontario)}

Background: Neuroleptic medications are recognized to produce a variety of delayed-onset neurological syndromes that may persist after discontinuation of the drug. Among these are tardive dyskinesia, tardive dystonia and tardive akathisia. We present clinical and experimental evidence that neuroleptics may produce tardive parkinsonism. Case: A 37-year-old woman with no past neurological or psychiatric history developed anxiety and insomnia. She was treated for 6 months with a depot neuroleptic, Modecate, in dosages 7-10x higher than are typically used in schizophrenia. 
When we first saw her 6 months after discontinuation of neuroleptics, she had marked parkinsonism, as well as akathisia and tardive dyskinesia. She still had clinical evidence of parkinsonism 18 months after stopping neuroleptics. Laboratory study: Rats were treated with daily injections of saline or neuroleptic (haloperidol $1 \mathrm{mg} / \mathrm{kg} /$ day) for 8 weeks, then sacrificed 2 weeks after the final injection. Animals in the haloperidol-treated group had $20 \%$ reduction in the cross-sectional area of the substantia nigra $(\mathrm{p}<.0001)$ and $32-46 \%$ fewer dopaminergic cell bodies $(\mathrm{p}<$ .01 ) as identified by tyrosine hydroxylase immunohistochemistry. Conclusion: These observations suggest that neuroleptic medications may be toxic to the dopaminergic neurons of the substantia nigra. Even relatively short-term neuroleptic treatment can result in dopaminergic cell loss in rats and persistent parkinsonism in humans.

\section{E-6}

\section{Limbic-Musical Biofeedback in Parkinson's Disease}

I.E. Szpindel, H. Chertkow, C. Melmed, L. Beaudet, D. De Kerckhove, J. Kraemer and M. Freedman (Hampstead, Montreal, Quebec; Toronto, Ontario)

Patients with Parkinson's disease are known to show preserved motor responses in the presence of limbic stimuli. This suggests that primitive limbic motor pathways remain potentially accessible via other stimuli such as music. We have adapted a computer based system which attempts to utilize these pathways through the creation of musical biofeedback representative of patient movement.

As a pilot study patients with Parkinson's disease were subjected to musical biofeedback sessions for 6 weeks. Three patients received true biofeedback and three patients received sham biofeedback as a control. Motor function was measured according to a modified UPDRS scale with patients and assessors blinded as to use of sham or real biofeedback. At midpoint one patient showing improvement from the treatment group was crossed over to placebo and a matched patient from the placebo group showing no improvement was crossed to treatment. At endpoint the patient crossed to control had deteriorated but remained improved over baseline. The patient crossed to treatment had improved. One other treatment patient showed significant motor improvement at endpoint. The final treatment patient and remaining control patients showed minor improvements only.

Results of this pilot study suggest that musical biofeedback may serve as an effective rehabilitation technique to improve motor function in some patients with Parkinson's disease.

\section{F. NEUROGENETICS $(F-1$ to $F-5)$}

(Thursday, June 22, 14:00 - 15:15)

F-1

The Oculopharyngeal Musculary Dystrophy Locus Maps to the Region of the Cardiac $\alpha$ and $\beta$ Myosin Heavy Chain Genes on Chromosome 14q.

Bernard Brais, Y. Xie, M. Sanson, K. Morgan, A. Korcyzn, S.C. Blumen, Ramat Aviv, J. Weissenbach, M. Fardeau, F.M.S. TOMÉ, J.-P. BOUCHARD and G.A. Rouleau (Montreal, Quebec, Quebec; Israel; Paris, France)

Oculopharyngeal muscular dystrophy (OPMD) is a late onset autosomal dominant muscular dystrophy which presents typically after the age of fifty with progressive eyelid drooping and increasing difficulty swallowing. Though OPMD has a worldwide incidence, it is more common in the French Canadian population. We have identified a homogenous group of families and studied 166 polymorphic markers as part of a genome search before establishing linkage to chromosome 14 . We determined that the OPMD locus maps to a less than $5 \mathrm{~cm}$ region of chromosome $14 \mathrm{q} 11.2-$ q13. The maximum two-point lod score in three French Canadian families of $14.73(\theta=0.03)$ was obtained for an intronic cardiac $\beta$ myosin heavy chain gene ( $M Y H 7)$ marker. The regional localization for the OPMD locus raises the intriguing possibility that either the caridac $\alpha$ or $\beta$ myosin heavy chain genes may play a role in this disease.

\section{F-2}

Correlation of Imaging Findings, Clinical Course, and Trinucleotide Repeats in Huntington's Disease Patients

M. Lewall, R.J. Sevick, O. Suchowersky, L. Graham, M.L. KLIMEK and A. GARBER (Calgary, Alberta)

In Huntington's disease (HD), a progressive neurodegenerative disorder, imaging studies have revealed atrophy of the caudate nucleus, previously documented by a number of different measurements. We investigated patients with HD to determine correlation between these measurements, clinical course and trinucleotide DNA repeats.

Imaging studies (CT, MR) were retrospectively reviewed in 28 symptomatic HD patients and 75 controls. Several parameters were used to measure caudate atrophy: intercaudate/inner skull diameter (CC/IT), intercaudate/frontal horn diameter, and intercaudate/outer skull diameter. Clinical charts were reviewed on all patients. Trinucleotide repeats were available in 22 patients.

CC/IT was found to have the best correlation with duration of observable symptoms, revealing exponential atrophy of the caudate nucleus with increasing duration of symptoms. Extrapolation from a graph of CC/IT vs. duration of symptoms suggested that there was a threshold beyond which patients began to show symptoms; there was a significant difference in CC/IT between HD patients and controls present at time of symptom onset. Correlation of CC/IT symptom duration vs. number of DNA repeats showed a greater amount of atrophy per unit time with increasing DNA repeats. This study suggests that significant caudate atrophy is present at time of symptom onset, and rate of atrophy is greater with larger number of DNA repeats.

\section{F-3}

\section{Cognitive Abilities in Huntington's Disease in Relation to} Clinical Onset and Genetic Analyses

G.W. Jason, O. Suchowersky, E.M. Pajurkova, L. Graham, M.L. KLIMEK and A. GARBER (Calgary, Alberta)

Neuropsychological evaluation was administered to 50 people with Huntington's Disease and 127 people at risk for the disorder. Thirty-one clinically affected and 86 at-risk people also had molecular genetic analysis. Significant cognitive decline was seen in subjects with young onset as well as late onset. In clinically affected subjects, cognitive ability was worse in those who had been affected for a longer time. With higher numbers of trinucleotide repeats in the abnormal gene, clnical onset occurred at a younger age. The rate of cognitive decline was examined by dividing the measure of cognitive ability by the number of years affected. Results were consistent with either cognitive decline 
beginning before clinical onset, or faster deterioration in early stages of disease, or both. In clinically normal subjects with abnormal genetic results, cognitive ability was worse in those with a higher number of trinucleotide repeats. This may reflect the beginning of cognitive deterioration in clinically normal people with a high number of trinucleotide repeats, who will go on to develop Huntington's Disease at a younger age than those with fewer repeats.

\section{F-4}

Susceptibility to Multiple Sclerosis: Linkage in HLA DR2+ Families but not DR2- Families

S.S. Beall, M. Hockertz, H. McFarland, W.E. Biddison, P. DuquetTe and G.Thomson (Vancouver, British Columbia; Bethesda, Maryland; Montreal, Quebec; Berkeley, California)

To test for linkage of susceptibility to multiple sclerosis (MS) and the HLA locus, affected sib pair analysis (ASP) of haplotypes identical by descent (IBD) was utilised in 66 ASPs from the United States $(n=32)$, British Columbia $(n=13)$, and Quebec ( $=21$ ). Evidence for heterogeneity of sharing was found in ASPs from Quebec vs. American and British Columbian families ( $p<$ 0.05 ). In families in which there was more than one ASP per family evidence for linkage was found in the American families ( $p$ $=0.025$ ) but not the French-Canadian families which conversely showed a non-significant $(p=0.37)$ whift towards sharing of 0 haplotypes IBD. Linkage with the HLA locus and susceptibility to MS was found in DR2+ non French-Canadian families when the DR2 allele was maternally derived $(p=0.045)$ but not paternally derived $(p=0.55)$. Linkage was not found in French-Canadian families or in DR2- non French-Canadian families. The data exclude linkage of the HLA gene locus with susceptibility to MS unless maternally derived risk alleles are taken into account. The data show linkage of this locus with susceptibility in non FrenchCanadian DR2+ families if the risk allele is maternally derived. Futher studies should examine the influence of maternally derived risk alleles upon susceptibility.

\section{F-5}

\section{Mitochondrial DNA Depletion: Estimation of Prevalence in a} Pediatric Population Referred for Neurological Evaluation

\section{C.J. MaCmillan and ERIC A. ShEnbridge (Montreal, Quebec)}

Mitochondria are unique organelles in that their biogenesis depends on the expression of both nuclear and mitochondrial genes. A new mitochondrial disease, mtDNA depletion, has been described in which there is a quantitative error of mtDNA copy number. A nuclear encoded defect is suspected as there is no evidence of maternal inheritance, and no miDNA point mutation or deletion has been found. To estimate the prevalence of mtDNA depletion in children referred for neurological evaluation, we quantitated the mtDNA: 18 sribosomal DNA ratio in muscle biopsy samples from all children with compatible clinical histories referred from the Montreal Children's Hospital between 1983 and 1994. Inclusion criteria were: age under 2 years at onset of disease and weakness, hypotonia or developmental delay. Exclusion criteria were: muscle destruction or evident histological diagnosis. 304 biopsies were evaluated, of which $68(22 \%)$ met the study criteria. We found 4 cases of mtDNA depletion, or $6 \%$ of those meeting the study criteria and $1.3 \%$ of the total biopsies. Further delineation of the phenotype at the cellular level is underway using in situ hybridization and immunocytochemistry. Evaluation for mtDNA depletion should be included in the workup of children with unexplained weakness, hypotonia or developmental delay.

\section{G. PEDIATRIC NEUROLOGY $(G-l$ to $G-5)$}

(Thursday, June 22, 15:30-16:45)

\section{G-1}

\section{Acute Swelling of the Cerebellum in Childhood}

\section{J. Hukin, D.D. CoChraNE and J.U. CRICHTON (Vancouver, British Columbia)}

Acute cerebellar swelling, severe enough to cause concern about herniation, is rare in childhood. The few reported cases emphasize its serious nature. We report two cases illustrating problems of diagnosis and treatment, but with good outcome.

Case 1. Male, 11 years, had a 2 day history of progressive occipito-frontal headache with vomiting, phonophobia and photophobia. The only positive finding was an equivocal right plantar response. A day later, he developed a mild left 6 th and 7th cranial nerve palsy. CT and MR imaging showed bilateral cerebellar swelling with evidence of upward and downward herniation. $\mathrm{He}$ was treated with dexamethasone over a 5 day period and recovered uneventfully.

Case 2. Female, 10 years, with a history of recent mild head injury, developed over six days headache, vertigo, diplopia and ataxia. She had a mild right 7 th nerve palsy, right sided palatal weakness and mild cerebellar signs. CT and MR imaging showed swelling of cerebellum and meningeal enhancement with obliteration of ambient cisterns. She recovered well with conservative therapy.

The search for infectious agents was unfruitful although the clnical course suggests a para-infectious etiology. These cases, managed conservatively, contrast with the poor outcome in most reported cases.

\section{G-2}

\section{Brain Herniation in Children with Cerebral Dysgenesis}

K.A. Selby, K. Farrell and A. Hill (Vancouver, British Columbia)

We describe four children with cerebral dysgenesis and epilepsy who became comatose and developed clinical and neuroradiological evidence of brain herniation in the absence of status epilepticus.

The underlying diagnoses were hemimegalencephaly, Pfeiffer syndrome, hypomelanosis of Ito with hemihypertrophy, cerebral dysgenesis with absent corpus callosum and polymicrogyria. Two children with ventriculo-peritoneal shunts had no evidence of shunt malfunction.

All children presented with a history of frequent seizures, decreasing level of consciousness and brainstem dysfunction. EEG's performed during the clinical deterioration demonstrated no evidence of status epilepticus. CT scans demonstrated effacement of the basal cisterns in all patients. The two patients with radiologic evidence of cerebral edema died. There was cerebral asymmetry in two of the children and craniosynostosis in another child.

We speculate that increased seizure activity may have contributed to brain herniation, possibly by increasing cerebral blood volume in children with compromised intracranial space. Patients with frequent seizures and cerebral dysgenesis may be at increased risk for cerebral herniation. 


\section{G-3}

Treatment of Guillain-Barré Syndrome with Intravenous Gammaglobulin in Children

L.A. Castagna, J. Vajsar, E.G. Murphy, R.I. Munn and A.E. SloANE (Toronto, Ontario)

The clinical course of 24 children (gange 3-16, mean 10.3 years) with Guillain-Barré Syndrome (GBS) treated with highdose intravenous gammaglobulin (IVIG) was evaluated retrospectively. On admission, the patients were either bed-ridden, or could walk only with support. $15(62 \%)$ had bulbar involvement, 8 (33.3\%) had autonomic symtoms; 5 patients $(20.1 \%)$ required ventilatory support for an average of 19 days. Complications were documented in 9 patients (37.5\%), including vomiting (3), headache (2), pneumonia (2), sepsis (2), fever (1), urticaria (1), and deep venous thrombosis (1). No patient deteriorated after administration of IVIG; all but two $(8.3 \%)$ recovered full function. There was no mortality.

In comparison, combining the data of several studies published after 1979, in untreated children the incidence of respiratory failure was $14 \%$, with an average time of ventilation of 29 days; the incidence of incomplete recovery or recurrent GBS was $8.4 \%$, and the mortality $2 \%$.

Our observations suggest that IVIG is a safe treatment which may reduce duration of assisted ventilation and mortality in children with GBS.

\section{G-4}

\section{X-Linked Adrenoleukodystrophy - an Expanding Clinical} Spectrum

F.A. Booth, S.S. Seshia, S.J. Bow, M.L. Schroeder and C.R. GrEenBERG (Winnipeg, Manitoba)

Adrenoleukodystrophy (ALD) is an $X$-linked peroxismal disorder characterised by central nervous system inflammation and demyelination and adrenal insufficiency. We reported on 4 affected boys and their extended families. Patient 1 presented at 10 years with acute transient visual loss. Optic neuritis was suspected. Mother and sister have neurological dysfunction felt secondary to toxic exposure but are ALD heterozygotes. Patient 2 presented at 11.5 years (when ALD diagnosed in cousin) with apparently static neurological dysfunction. Mother was considered to have "multiple sclerosis" (MS) but is an ALD heterozygote. Patient 3 presented at 5.5 years with incoordination and visual impairment. His maternal uncle with known adrenal insufficiency has the biochemical defect of ALD and mild adrenomyeloneuropathy. Patient 4 presented at 3.5 years with mild speech delay and acute transient left hemiparesis, then months later with acute right hemiparesis progressing to quadriparesis with rapid recovery. Maternal grandfather had a past diagnosis of MS but is an ALD hemizygote. Four other male members are affected, some asymptomatic. We conclude that the presentation of ALD may be acute and transient or seemingly static and we emphasize the need to screen for unrecognized or asymptomatic cases within affected families.

\section{G-5}

On the Prevalence and Clinical Features of Tuberous Sclerosis: A Population Study

D.W.M. WeBB, A.E. FrYer and J.P. Osborne (Vancouver, British Columbia; Liverpool, Bath, England)

The frequency of clinical features and disease complications of tuberous sclerosis (TSC) is derived largely from institution based studies, hospital series and small population cohorts.
We sought to identify individuals with TSC who were resident in an area with a known population. Specialists likely to encounter individuals with the disease were contacted. A full clinical evaluation was performed on index cases and their first degree relatives.

131 individuals with TSC (aged 6 months to 74 years) were living in the study region giving a total population prevalence of $1 / 26,500$. The prevalence was highest in children under 5 years $1 / 11,600$. The reasons for presentation were: seizures in $90(69 \%)$, identification of the family proband in $21(16 \%)$, skin lesions in 10 $(8 \%)$, giant cell astrocytomas in 3 , renal angiomyolipomas in 3, polycystic kidney disease in 2 and unknown in 2 .

The prevalence of cutaneous lesions varied widely with age. Retinal astrocytomas were detected in $24 \%$, macrocephaly in $40 \%$ of affected children, seizures (at some point) in $78 \%$ and mental handicap in $53 \%$. The age of onset of seizures and risk of mental handicap were related. The birth incidence of TSC is at least $1 / 12,000$. The prevalence of neurological sequelae is lower than estimated from hospital based series.

\section{H. NEUROSURGERY - TRAUMA ( $H-1$ to $H-5)$}

(Friday, June 23, 10:30-12:00)

\section{H-1}

Predicting Mortality From CT Head Scans in Head Injured Patients

Magdy S. Shady, M.I. Khan and S. Lal (Stony Brook, New York; Saskatoon, Saskatchewan)

Seventy-seven patients with significant head trauma were admitted to University Hospital in Saskatoon. CT scans were obtained on inpatients with Glasgow Coma Score of 8 or less. Surgical lesions were treated immediately after scanning. This study examines the relationship between the radiographic findings observed on the CT scans and mortality in severe head injured patients in an attempt to identify radiological findings that will independently predict mortality caused by head injury. The most common abnormal CT findings on admission in descending order or frequency included abnormal lateral ventricles, subdural hematoma, intracerebral hematoma, obliterated cistern, shift of midline structures and subarachnoid hemorrhage. The relationship between mortality and both radiographic and clinical findings were examined. The independent effects of these factors were then evaluated using logistic regression. Significant CT findings include subdural hematoma, intracerebral hematoma, abnormal third ventricle, complete cisternal obliteration, septal shift greater than 15 $\mathrm{mm}$ and pineal shift greater than $5 \mathrm{~mm}$. Clinically, a GCS of less than 8 and dilated pupils are both significantly associated with high mortality rate. Statistically, it would appear that only a GCS less than 8 , complete cisternal obliteration, intracerebral hematoma and SAH were independent predictors of outcome.

\section{H-2}

Electroencephalography, Somatosensory Evoked Potentials and Cerebral Blood Flow after Traumatic Brain Injury in Humans

J.I.M. Brown, S.J. Konasiewicz, A. Baker and R.J. Moulton (Toronto, Ontario)

Introduction: Cerebral ischaemia is believed to be an important cause of secondary damage following traumatic brain injury (TBI). Abnormal electrophysiologic function may be observable 
at levels of ischaemia that do not cause frank infarction. We examine relationships between $\mathrm{CBF}$ and quantitative EEG and SSEP indices in the acute period following TBI to look for associations between brain electrical activity and cerebral blood flow.

Methods: From July 1987 to January 1995, intensive monitoring of 36 patients with severe TBI (GCS $\leq 8$ after resuscitation) included continuous EEGs, hourly SSEPs, and twice daily CBF $\left(\mathrm{N}_{2} \mathrm{O}\right.$ inhalation method) for an average of five days. EEGs were quantitated as total spectral power (TSP) and percent slow wave activity (Delta) and SSEPs as summed peak to peak amplitudes. Data were assessed by correlation analysis and analysis of variance.

Results: Changes in Delta and SSEP over the monitoring period (CDelta and CSSEP) showed significant correlation with CBF ( $r=0.219, p=0.009 ; R=-0.240, p=0.0012$ respectively) which was strongest in the early period ( $<48$ hours) post injury ( $R$ $=0.493, p=0.0001 ; R=-0.535, p=0.0001$ respectively) and in patients with poor outcomes $(R=0.285, p=0.0001 ; R=-0.271, p$ $=0.0007$ ). Patients with CBF $\leq 40 \mathrm{ml} / 100 \mathrm{~g} / \mathrm{min}$ differed significantly in initial Delta, initial SSEP, CDelta, CTSP, and CSSEP from those with flows over 40 . No electrophysiologic index showed significant differences between blood flow classes when blood flows were further stratified into normal, high, and reduced flow categories.

Conclusions: Changes in Delta and SSEP are correlated to CBF, especially in the early period, following severe TBI. CBF levels not usually considered ischaemic are associated with significant electrophysiologic aberrations after severe TBI. CBF thresholds may be altered in this population.

\section{H-3}

\section{Chronic Subdural Hematoma Revisited; an Analysis of Surgical Outcome}

B.H. Guiot, B.G. Benoit, H.J. Lesiuk, M.T. RiCHARD, S.D. MCCluskeY and R. BENOIT (Ottawa, Ontario)

A restrospective review of 184 patients with chronic SDH treated at 2 adult teaching hospitals between 1987-1994 was conducted. Demographics between the burr hole $(n=91)$ and craniotomy $(n=93)$ cohorts were similar, as was the eventual surgical outcome. Re-operation was necessary in 41 patients $(22 \%)$, an incidence at the high end of the reported range. Hematoma volumes were calculated from direct $\mathrm{CT}$ scan console reconstruction or using a square grid analysis of hard copy. Hematoma volumes in the 2 surgical groups were similar.

Re-expansion intraparenchymal hemorrhage occurred in 4 patients $(2 \%)$ and will be the object of a subset analysis.

Both the procedure time and hospital length of stay were shorter in the burr hole group. Preliminary data indicates that significant recurrence is more likely in large volume hematomas, and the need for repeat surgery is more likely if the initial hematoma was large and was treated by craniotomy. These preliminary results suggest that there is a need for prospective trial comparing the outcome of the two surgical techniques.

\section{H-4}

\section{Decompressive Craniotomy and Duraplasty in the Treatment of Traumatic Brain Swelling}

\section{MEHTA and R.O. HolNESS (Halifax, Nova Scotia)}

Decompressive craniotomy has been abandoned in many centers for the treatment of patients with head trauma. This is largely due to the high incidence of vegetative survivors in the reported series of patients with acute subdural hematoma (and poor Glasgow Coma Scales) who were treated with this method.

We had used decompressive craniotomy and duraplasty in a select group of patients with intractable intracranial hypertension, ominous brain shift on CT, and in whom the main pathology was unilateral cerebral swelling.

We present five such young males whose intracranial pressure did not respond to hyperventilation, sedation, mannitol and external ventricular drainage. CT scans showed diffuse brain swelling, worse on one side, with shift and compression of the basal cisterns. All patients wre treated medically for a minimum of 48 hours before operative treatment. All five patients survived in good condition. Three were university students at the time of their injury and were able to return to school. They all display varying degrees of residual neuropsychological deficit but function independently.

On the basis of this experience, we conclude that decompressive craniotomy and duraplasty is worth considering in patients with unilateral cerebral swelling who do not respond to medical measures. A favourable Glasgow Coma Scale on admission correlates with the quality of survival and is the critical criterion in selecting patients for this avenue of treatment.

\section{H-5}

\section{The Course of Post Traumatic Amnesia}

M.L. Schwartz, D.T. Stuss, F. Carruth, C. Brandys, R. MOULTON, G.W. SNOW and M.A. Binns (Toronto, Ontario)

Ninety-one patients with traumatic brain injuries were examined repeatedly following injury with the Galveston Orientation and Amnesia Test (GOAT) and a test of their ability to learn and retain new information. Three concrete words of high frequency were presented. The subjects were asked to look at each work individually for 10 seconds and to read it aloud. Immediate and 24hour recall were tested. If 24-hour recall was imperfect, recognition was tested by presenting 9 words including the 3 target words and 6 distracters. The words were re-presented and recall was tested at intervals after 24 hours. The time intervals to first recognition and first free recall were compared with the patients' first GOAT score of 75 to see whether a score of 75 accurately predicted the patients' ability to learn and recall new verbal information.

For all categories of head injury severity, the median interval to perfect free recall followed the achievement of a GOAT score of 75 by a significant interval. The majority of patients with minor brain injuries could not pass the 3 -word recognition memory test until significantly after the GOAT score was 75 , whereas, in patients with moderate and severe injuries, recognition preceded a GOAT score of 75 by a significant interval.

If ability to recognize and/or recall 3 words signifies emergence from post-traumatic amnesia (PTA) then a GOAT score of 75 does not identify it. Three-word recall, which is simpler to administer than the GOAT, is a more reliable measure of emergence from PTA.

\section{NEUROLOGY - GENERAL (I-I to $I-15)$ \\ (Friday, June 23, 10:30 - 12:00)}

\section{I-1}

The Syndrome of Subacute Diabetic and Uremic Polyneuropathy

C.F. Bolton, M. McKeown, R. Chen, H. Remtulla, L. BERNARDI, B. TOTH and R.M. LindSAY (London, Ontario)

Combined diabetic and uremic polyneuropathy is being observed with increasing frequency. It is a difficult problem in 
diagnosis and treatment, partly because it is difficult to determine which disease contributes to the polyneuropathy. We describe four adult patients, observed in the last four years, who had distinctive features. They had recently been treated by chronic hemodialysis for end-stage renal disease. Progressive, moderate to severe weakness of proximal and distal limb muscles developed over several months. Comprehensive electrophysiological studies indicated a primary axonal motor and sensory polyneuropathy with prominent secondary segmental demyelination. There was unusual paucity of abnormal spontaneous activity on needle electromyography, possibly due in part to the effect of uremic toxins (Buront et al., 1991). Muscle biopsy showed features consistent with denervation atrophy. The dialysis techniques were changed to remove middle molecules with resulting improvement in the neuropathy in three patients, suggesting renal failure was the main cause of the polyneuropathy.

Thus, subacute diabetic and uremic polyneuropathy may represent a syndrome with distinctive, but potentially reversible, features.

\section{I-2}

Clinical Efficacy and Cost-effectiveness of Long Term Intrathecal Baclofen Therapy in Patients with Severe Spasticity

W.J. Becker, C.J. Harris, M.L. LonG, D.P. Ablett, G.M. Klein and D.A. DeForge (Calgary, Alberta; Ottawa, Ontario)

An analysis of clinical efficacy and cost-effectiveness was done for intrathecal baclofen (IB) therapy in all patients treated with IB therapy at our centre in whom one year or more of follow-up after initiation of therapy was available. Six patients had multiple sclerosis, two cervical spinal cord injury and one head injury. All were non-ambulatory.

Patients showed improvement in many areas, including ability to transfer, seating, pain control, personal care and liability to skin breakdown. Before IB therapy, only three of the nine patients were able to live at home in the community and six were institutionalized. At the end of our follow-up, only one patient remained institutionalized, three lived in group homes, and five lived at home in the community. In the year preceding pump implantation the nine patients spent a total of 755 days in acute care hospitals; in the year following only 259 days. Complications of therapy and a cost analysis of treatment will be presented in detail.

IB therapy can improve patient quality of life and can be costeffective in carefully selected patients with severe spasticity and disability. The drug delivery catheter is that part of the therapeutic system most vulnerable to failure.

\section{$1-3$}

\section{Seizures Triggered During Love-Making (Ictus Eroticus)}

\section{A.O. OGUNYEMI and JAMES WoODRow (St. John's, Newfoundland)}

Very little information exists concerning the precipitation of seizures during sexual activities. This report describes 3 patients who consistently experienced epileptic seizures during sexual engagement with their partners.

Two patients are female aged 25 and 28 years; the third is a male aged 26 years. All of them suffer from complex partial seizures with or without secondary generalization. In addition, the female patients experience simple partial seizures with epigastric (visceral) or somatosensory sensation. The female patients began to have seizures at ages 4 and 7 years and were never free of seizures for more than a few months. As adults, they had seizures during sexual activities and also when they were not making love. For the male patient, seizures began at age 10 years and remained recurrent for 2 years. Thereafter, he was seizure-free until age 23 years when he began to have seizures related only to love-making.

In the 3 patients, the complex partial seizure consisted of abrupt loss of contact, vacant stare, kissing motions and protrusion of the tongue. The immediate post-ictal manifestations included a brief smile followed by irritability, swearing and sex-seeking behaviour. EEG recordings were normal in the male patient but showed asynchronous bitemporal spikes and sharp waves in the female patients.

\section{I-4}

\section{Catatonia Following Withdrawal of Benzodiazepine Medication}

P.I. Rosebush and M.F. MAZUREK (Hamilton, Ontario)

Background: The use of benzodiazepine medication is associated with a variety of acute withdrawal syndromes that can command neurological attention, including seizures, confusion, and agitation. While these hyperarousal states are well recognized, it is not commonly appreciated that benzodiazepine withdrawal can produce psychomotor retardation. Method: Six patients $(2 \mathrm{M}, 4 \mathrm{~F})$ developed retarded catatonia following withdrawal of benzodiazepines. All 6 were older individulas (53-88 yrs) who had acutely become bed-ridden, immobile, mute and rigid, with refusal or inability to eat or drink. The diagnosis of catatonia had not been recognized in any of the patients prior to their being referred to us. Results: We treated each patient with lorazepam 1-2 mg., a regimen which we have previously reported to be effective in the treatment of catatonia. All 6 showed pronounced improvement within an hour, and complete reversal of the catatonic signs within 24 hours. Careful review of the records showed that each of the 6 patients had taken benzodiazepine medication for anywhere from 6 months to 15 years, but had stopped receiving benzodiazepines 2-7 days prior to developing catatonia. Conclusions: Severe and potentially life-threatening catatonia, presenting as an acute neurological illness, can develop in the wake of benzodiazepine withdrawal, particularly in older individulas. Reinstitution of benzodiazepine medicaiton can lead to dramatic resolution of the syndrome.

\section{I-5}

Reversible Neurological and Psychiatric Abnormalities in Adult $\mathrm{GM}_{2}$ Gangliosidosis: the Role of Neuroleptic Withdrawal and Benzodiazepine Treatment

\section{P.I. Rosebush and M.F. MAZureK (Hamilton, Ontario)}

Background: The neuropsychiatric abnormalities associated with adult $\mathrm{GM}_{2}$ gangliosidosis (AGG) are thought to be irreversible. After discontinuing neuroleptics and instituting benzodiazepine therapy, we have observed sustained neurological and psychiatric improvement over 18 months in a paitent with biochemically and genetically confirmed AGG. Methods: We identified severe-hexosaminidase A deficiency in a 17-year-old non-Jewish boy with a 6-year history of cognitive and motor deterioration, and a 3-year history of neuroleptic-resistant catatonic schizophrenia. Neurological signs included unintelligible speech, severe dysphagia with cachexia, nystagmus, dystonia, hyperreflexia, ataxia and inability to write or draw. Neurobehavioural abnormalities included poor concentration, marked perseveration, 
psychosis and bizarre behaviour. Treatment consisted of stopping neuroleptics and starting treatment with the benzodiazepine lorazepam. Results: The dysphagia, dystonia, nystagmus and hyperreflexia resolved over 3 months. At 1-year he was able to perform tandem gait and his speech was clearly intelligible. At 18 months he was able to write legibly, draw and complete a neuropsychological assessment. Conclusions: I. Neuroleptics, which are known to promote lipidosis, may exacerbate conditions such as AGG. Benzodiazepines may play a useful role in the treatment of AGG.

\section{J. CLINICAL NEUROPHYSIOLOGY ( $J-1$ to $J-5)$}

(Friday, June 23, 10:30-12:00)

\section{$\mathbf{J}-1$}

The Correlation Between Surface Electro-myography and Biochemical Tests to Measure an Athletes Performance

B.M. Chaudhuri, Stuart Donaldson and David SMith (Calgary, Alberta)

Various characteristics of the surface electromyography (sEMG) signal were examined in relation to known biochemical measures in the blood (creatine kinase, inorganic phosphate, etc.). Seven fully informed male volunteers participated in the study where the subjects completed an exhastive workout of the legs, followed by repeated measurements taken a) immediately after, b) $1,3,6,9,24$ and 48 hours post workout. Independently of the measurements, the subjects compelted a maximal assessment. Neurological and serological measurements were then taken at $0,1,3,6,9,24$ and 48 hours post workout. The workout consisted of knee extensions of 300 degrees $/ \mathrm{sec}$., $180 \mathrm{~d} / \mathrm{s}, 30 \mathrm{~d} / \mathrm{s}$ and an isometric contraction held at 30 degrees (the first two movements were repeated only twice due to severe strain). A measurement of the patient's potential physical ability was assessed. The interaction of the sEMG measures of the vastus lateralis and medialis were found to vary with time.

Preliminary results indicate low variability between experiments with regard to maximum amplitude measured both neurologically and serologically. As achieved by this report, sEMG measurements correlate quite closely with serological results indicating a neurological means of assessing an athletes ability to recover from a workout effectively.

\section{$\mathbf{J}-\mathbf{2}$}

Needle EMG of the Human Diaphragm: Power Spectral Analysis in Normal Subjects and in Patients with Respiratory Failure

R. Chen, H. Remtulla, K. Power, S. Collins and C.F. Bolton (London, Ontario; Victoria, Australia)

Needle EMG of the diaphragm was performed in 43 diaphragms in 23 healthy volunteers and in 14 patients with respiratory failure secondary to neuromuscular disease. The mean \pm standard deviation of the median frequency (MF) of the power spectrum was $233.3 \pm 58.1 \mathrm{~Hz}$ for the normal subjects. The MF increased with age and showed a negative correlation with the forced vital capacity (FVC). The higher MF in older subjects may be due to early recruitment of larger units. The negative correlation between MF and FVC is likely secondary to a lower level of contraction required for normal breathing in subjects with high FVC. The integrated EMG (iEMG) of each inspiration strongly correlated with the tidal volume and the duration of inspiration. The precise position of the needle within the diaphragm did not significantly affect the MF. Preliminary studies in patients with neuromuscular respiratory failure showed that the MF can be high, normal or low in patients with neuropathy and may be elevated in patients with myopathy. Decrease in the MF with time during spontaneous respiration may be associated with difficulty weaning from the ventilator. Power spectral analysis of diaphragmatic EMG is feasible and reliable. It is potentially useful in the assessment of respiratory muscle fatigue and may help in the diagnosis of neuromuscular disorders affecting the diaphragm.

\section{$\mathbf{J}-3$}

Electrophysiologic Features of Cervical Radiculopathy: a Population Based Study

M. Mosewich, K. Radhakrishnan and W. Litchy (Rochester, Minnesota)

Cervical radiculopathy is a common condition with an average annual age-adjusted incidence of 83.2 per 100,000 population. The clinical syndrome is well described but there are no detailed reports of the electrophysiologic observations in a large group of cervical radiculopathy patients using population based studies.

We studied 561 patients with cervical radiculopathy ascertained using the Mayo Clinic computerized medical linkage system to identify residents of Rochester, Minnesota between 1976-1990. Thirty-one percent (175) of these patients had electrophysiologic (EP) studies including electromyography and nerve conductions. The mean age of these patients was 49.4 years, 99 males and 76 were female. There were no statistically significant differences in operative versus non-operative treatment and functional outcome between EP group (EPS+) and the group without EP studies (EPS-). The EPS+ group were older (49.4 years vs. 46.5 years) and had an insidious onset of symptoms with more poorly localized pain in an upper extremity. In addition, they complained of weakness more frequently, they were more likely to have a C8 distribution and often had magnetic resonance imaging study abnormalities. These results and the detailed EPS findings of the EPS+ group will be presented.

This population based report provides useful information on the role of EPS in the evaluation of patients with cervical radiculopathy.

\section{J-4}

The Value of Diaphragmatic Needle EMG and Phrenic Nerve Conduction in Patients with Guillain-Barré Syndrome

U. Zifko, R. Chen, H. Remtulla, W. Wihlidal, A. Hahn, W. KOOPMAN and C.F. BOLTON (London, Ontario)

We determined the value of diaphragmatic needle electromyography (EMG) and phrenic nerve conduction by comparing these results with forced vital capacity (FVC) and the need for mechanical ventilation in patients with Guillain-Barré syndrome (GBS).

The results, mean \pm S.D., in 18 patients ( 12 male, 6 female, mean age 56) were compared to 25 healthy persons (mean \pm S.D.). Phrenic nerve parameters were abnormal in 12 patients $(66 \%)$ with a mean latency of $8.7 \mathrm{~ms} \pm 1.5 \mathrm{~ms}(6.5 \pm 0.8)$ and an amplitude of $460 \mu \mathrm{V} \pm 290 \mu \mathrm{V}(660 \pm 201)$. The diaphragmatic EMG revealed a neurogenic pattern in 9 cases $(50 \%)$, with fibrillation potentials and positive sharp waves between inspiratory bursts, a decrease in the number of motor unit potentials during respiratory bursts, the remaining ones being polyphasic and of normal or increased 
amplitude. The FVC (at the day of EMG) was abnormal in 14 patients $(77 \%)$, ventilation being required in 7 patients $(39 \%)$. The diaphragm compound muscle action potential correlated significantly with the FVC $(p=.0009)$ and with the need for ventilation $(p=.0419)$. Six of the 7 patients $(86 \%)$ requiring mechanical ventilation had an abnormal diaphragm EMG with a significant correlation of $\mathrm{p}=.0156$.

Our results indicate both phrenic nerve conduction and diaphragmatic EMG is valuable in management of patients with GBS.

\section{$\mathbf{J}-5$} Distinctive Electrophysiological Features of Denervated
Muscle in Uremic Patients

C.F. Bolton, H. Remtulla, B.Toth, L. Bernardi and R.M. LINDSAY (London, Ontario)

We describe the clinical, electrophysiological and morphological features of muscle denervation in five uremic patients. These observations have not been made previously, except for a preliminary report by our group (Burton et al., 1991).

The patients were a young male with traumatic femoral neuropathy resulting in severe axonal degeneration of the femoral nerve, and four older patients with subacute diabetic and uremic polyneuropathy, all five on chronic hemodialysis. The following observations were made in the distribution of the involved nerve(s). There was severe paralysis and sensory loss in the patient with traumatic femoral neuropathy and moderate weakness and sensory loss in the patients with polyneuropathy. Compound muscle and sensory nerve action potentials were reduced or absent, and latencies mild to moderately prolonged. Needle electromyography usually showed absent, occasionally sparse fibrillation potentials and positive sharp waves, even though insertional activity was present and the numbers of motor unit potentials were markedly reduced. Muscle biopsy showed features consistent with denervation atrophy. These needle electrode abnormalities are unusual and suggest uremic toxins may inhibit the generation of denervation potentials in human muscle.

\section{K. PEDIATRIC NEUROLOGY $(K-1$ to $K-6)$ \\ (Friday, June 23, 10:30 - 12:00)}

\section{K-1}

Diagnostic and Therapeutic Advances in Birth Traumatic Phrenic Nerve Injury

U. Zifko, M. HarmanN, W. GirSCh, A. LischKa, W. Grisold and K. LiNDNER (Vienna, Austria)

Recent diagnostic and therapeutic experiences in 4 newborns with birth traumatic phrenic nerve injury are reported. All our cases were breech-delivered; in one case with forceps. Phrenic nerve lesions occurred once bilaterally, and three times unilaterally, right-sided. Accompanying signs of traumatic birth were seen in all our infants, consisting of severe asphyxia, upper brachial plexus lesions, clavicular and humeral fractures. Diaphragmatic paresis was suspected clinically, between the second and sixth days of life. At this time, chest $\mathrm{x}$-rays were misdiagnostic for detecting diaphragm paresis in 2 babies. However, ultrasound examination of diaphragmatic motion and phrenic nerve conduction studies turned out to be the diagnostic method of choice. In all newborns, mechanical ventilation was required for at least 16 days.
Spontaneous recovery occurred in 2 children within the first five months, and 2 became asymptomatic after operative treatment. One child with unilateral phrenic nerve injury and recurrent pulmonary infections improved after plication of diaphragm at an age of 4 months. Due to recent advances in peripheral nerve reconstruction, surgical revision and autologous nerve transplantations were performed successfully in the baby with complete bilateral phrenic nerve lesion. However, careful preoperative nerve evaluation is mandatory for defininte operative decisions.

\section{K-2}

Significance of Thalamic Hypodensity on Computed Tomography in the Asphyxiated Term Newborn

E.C. Rodriguez, K. Poskitt, E.H. Roland and A. Hill (Vancouver, British Columbia)

In asphyxiated term newborns, diffuse decreased tissue attenuation on computed tomography (CT) performed at age 3-5 days correlates with poor outcome. We describe another pattern of brain injury on CT characterized by low attenuation in thalamus/basal ganglia and relative preservation of cortex which is associated with poor outcome.

In an ongoing study of acute neonatal hypoxic-ischemic cerebral injury, 17 term newborns had CT abnormalities involving low attenuation of thalamus/basal ganglia and relative preservation of cortex. 11/17 infants had a history which suggested acute, "total asphyxia": e.g., cord prolapse (3), uterine rupture (1), torn umbilical vein (1), massive abruptio (1), severe bradycardia (5). Clinical abnormalities included cranial nerve dysfunction in all infants. Marked tongue fasciculation may be a useful clinical marker. All infants had seizures suggesting some cortical injury. Injury to other organs was common: renal (17), cardiac (8), hematological (4). Neurological outcome was poor: death (5), prolonged nasogastric feeding (6), multiple severe abnormalities (microcephaly, spastic quadriplegia, seizures) (9), hemiplegia (1).

These data demonstrate that a pattern of injury involving thalamus/basal ganglia and presumably brainstem with relative preservation of cortex can be recognized on CT and is secondary to acute, total asphyxia. The outcome of such insult in the term newborn is poor.

\section{K-3}

Possible Etiological Factors Associated With Increasing Incidence of Periventricular Leukomalacia

E.C. Rodriguez, A.Y. Manzur, E.H. Roland, B.A. Lupton and A. HILl (Vancouver, British Columbia)

Recent reports suggest an increase in the incidence of periventricular leukomalacia (PVL) in the premature newborn. We document the incidence of cystic PVL in premature newborns and identify possible etiological factors.

A computerized database was reviewed of premature newborns (BW < 1500g; $n=2,159$ ) admitted between 1985-1993. 61 infants had cystic PVL on serial ultrasound scans. Between 1985 and 1991, the incidence was 1-6/year (average: $2 \%$ ) which rose to $10 / 215$ in $1992(4.7 \%)$ and $16 / 209$ in $1993(7.7 \%$ ) without change in imaging surveillance. During this period, there was a major decrease in annual mortality rate from $20.4 \%$ (1985-1991) to $11 \%$ (1992-1993). Cystic PVL occurred before 4 days of age, suggesting antenatal origin, in 3/35 infants (3\%) between 1985-1991 and increased to $8 / 26$ infants $(30 \%$ ) in 1992-1993. Compared with a control population, significant etiological factors were multiple 
pregnancy $(p<0.011)$, maternal infection $(p<0.0001)$ and duration of ventilation $(p<0.007)$. The following did not reach statistical significance: sepsis, bronchopulmonary dysplasia, surfactant, steriod and colloid therapy, hypoglycemia.

This increasing incidence of cystic PVL may be explained by the major decrease in mortality rate of premature infants and by an increased incidence of multiple pregnancy. Maternal infection may contribute to increase in antenatal onset of PVL.

\section{K-4}

Acute Hypertensive Encephalopathy in Children - Clinical and Neuroradiological Findings

K.A. Selby, K. Poskitt and A. Hill ( Vancouver, British Columbia)

We describe six episodes of hypertensive encephalopathy in five patients aged $4-18$ years, characterised by headaches, seizures, visual changes, altered level of consciousness, and markedly elevated blood pressure.

Four had renal pathology, one had steriod resistant nephrotic syndrome, three had chronic renal failure and the other had Fanconi's anemia. One child had a renal transplant and one a bone marrow transplant prior to developing hypertensive encephalopathy. Four children were on steroids of which three were also on cyclosporine.

All had early CT scans. Eleven CT scans were performed, of which three were late follow up scans. Five early CT scans showed multiple foci of decreased attenuation in white matter most marked in parietal and occipital lobes, with little or no involvement of cortex. One early scan revealed a temporoparietal intracerebral hematoma and edema of the centrum semiovale. Three late CT scans demonstrated marked resolution.

Clinically all patients improved. The CT findings are consistent with reversible cerebral edema. The pathophysiology of these entities is difficult to determine. Disruption of cerebral autoregulation may be the mechanism of reversible cerebral involvement in hypertensive encephalopathy.

\section{$\mathbf{K} \cdot 5$}

\section{Functional MRI (fMIR) Localization of Language in Children}

W.J. Logan, R.R. Benson, G.R. CosGrove, B.R. BuChBINDER, H. JIANG, L.L. LeSueur, V.S. CAVINESS JR., B.R. Rosen and A. COLE (Charlestown, Boston, Massachusetts)

Localizing critical brain function prior to surgery generally requries invasive techniques. Activation PET imaging can localize function but is limited by the amount of radioisotope that can be administered, especially in children. MRI can detect focal changes in signal intensity associated with specific brain activity (fMRI) using fast imaging techniques. In addition to sensory and motor functions, language activity has been localized in normal adults and in patients with brain lesions (Benson et al., Soc. Neurosci. Abst. 9.7, 1994). Our goal was to determine if this approach could be adapted for the evaluation of language in children.

We studied a nine-year-old right handed male with attention deficit, mildly decreased verbal proficiency and intractable epilepsy who was scheduled for left temporal lobe surgery. Using fMRI, language was mapped on multiple sections through the whole brain using a $1.5 \mathrm{~T}$ GE imager equipped with echoplanar fast imaging. To optimize performance, increased interstimulus intervals and age appropriate tasks were used including covert word reading, verb generation, antonyms and letter fluency.

With instruction he was able to perform each task during the imaging. Two of the task activations were without motion artifact and revealed clear lateralized activity similar to that seen in normal adults. It was possible, therefore, to obtain reproducible brain activation with specific language tasks in this child. This non-invasive method for evaluation of language function appears to be feasible in children and should be helpful in presurgical localization of function.

\section{K-6}

Canadian Pediatric Ischemic Stroke Registry: an Updated Analysis

\section{G.A. Deveber (Hamilton, Ontario)}

Since January 1992 we have now identified 156 consecutive children $<18$ yrs (135 with complete information) with radiographically proven arterial ischemic stroke or sinovenous thrombosis at all tertiary care pediatric centres in Canada $(\mathrm{N}=16)$. We report an updated analysis of clinical and radiological features of this entity. The incidence was 1.00 per 100,000 children per yr. and was highest in infancy; neonates: $\mathrm{N}=33(24 \%)$; infants $>1$ mo. and < 1 yr: $\mathrm{N}=13(9 \%) ; 1-<5$ yrs: $\mathrm{N}=38(28 \%) ; \geq 5$ yrs: $\mathrm{N}=$ $53(39 \%)$. Males $(\mathrm{N}=77)$ were more frequently involved than females $(\mathrm{N}=58)$. Arterial involvement predominated; large vessel arterial: $\mathrm{N}=49(36 \%)$, small vessel arterial: $\mathrm{N}=53(39 \%)$ and sinovenous: $N=34$ (25\%) (53\% with venous infarction). Risk factors were: non identified: $\mathrm{N}=36(27 \%)$, cardiac disease: $\mathrm{N}=28$ (21\%), infection/inflammation: $N=21(15 \%)$, coagulation disorder: $\mathrm{N}=10(7 \%)$, dehydration: $\mathrm{N}=8(6 \%)$, dissection: $\mathrm{N}=7$ (5\%), metabolic: $\mathrm{N}=3(2 \%)$, Moya moya: $\mathrm{N}=3(2 \%)$, perinatal complications $\mathrm{N}=3(2 \%)$ and other known: $\mathrm{N}=16(12 \%)$. Outcome at discharge $(\mathrm{N}=120)$ was: $8(6 \%)$ dead, $68(57 \%)$ with neurologic sequelae and $43(36 \%)$ normal. Multiple or recurrent strokes occurred in $11 \%$. Treatment was variable but anticoagulants were used in $32(24 \%)$ patients with: sinovenous thrombosis $(43 \%)$, cardiac disease $(23 \%)$, dissection (13\%), multiple infarcts $(10 \%)$ and unknown indications $(7 \%)$. In conclusion pediatric cerebral thromboembolism is common, especially in infancy. The outcome and variability in treatment support the need for intervention trials in pediatric cerebral thromboembolism. (Funded by Bloorview Children's Hospital Foundation.)

\section{NEUROLOGY - GENERAL ( $L-1$ TO $L-11)$ \\ (Friday, June 23, 14:00-17:00)}

\section{L-1}

A Monoclonal Antibody to $\alpha 4$-integrin Reverses the Clinical, Histological and MRI-detectable signs of Experimental Allergic Encephalomyelitis in the guinea pig

S.J. Karlik, S.J. Hyduk, G.P.A. RiCE and H. HorNer (London, Ontario; San Francisco, California)

In experimental allergic encephalomyelitis (EAE) circulating leukocytes enter the central nervous system (CNS) leading to inflammation, myelin damage and paralysis. Leukocyte-endothelial adhesion, through the VLA4/VCAM-laxis at the blood-brain barrier, is considered an essential step in the mediation of CNS leukocyte infiltration in EAE. This study examined the ability of a monoclonal antibody to $\alpha 4$ integrin to reverse an acute EAE episode. AN $100226 \mathrm{~m}$ treatment during active disease produced a rapid clearance of leukocytes from the CNS which was accompanied by a reversal in clinical signs. Histologically, treated animals showed a marked decrease in the abnormal pathological signs 
associated with EAE, including demyelination. Magnetic resonance imaging is important for the diagnosis of multiple sclerosis (MS) and the evaluation of potential new therapies. Following AN100226m treatment in acute EAE, gadolinium enhancement of the CNS parenchyma was also significantly decreased indicating closure of the blood-brain barrier. A decrease in CNS abnormalities associated with cerebral adema and inflammation was also observed in T2-weighted images $(p<0.05$, Mann Whitney Rank Sum). Thus, an antibody to $\alpha 4$ integrin reversed the blood-brain barrier permeability changes characteristic of acute $\mathrm{EAE}$, as well as the clinical and histological signs of disease. We conclude that AN $100226 \mathrm{~m}$ is an effective treatment for EAE and may be similarily useful in the treatment of autoimmune diseases such as multiple slerosis.

\section{L-2}

\section{The Variability of Multiple Sclerosis Lesion Quantification}

S.J. Karlik, J.R. Mitchell, D.H. Lee, M. Eljiasziw, G.P.A. Rice and A. FEnSTER (London, Ontario)

Magnetic Resonance Imaging (MRI) has enabled non-invasive visualization and quantification of Multiple Sclerosis (MS) lesions in vivo. Nevertheless, accurate quantification techniques are subject to inter- and intra-operator variability, which may hinder monitoring of disease progression. We have developed a computer assisted technique for quantification of MS lesions in spin-echo MR exams. Inter- and intra-operator variabilities of assisted and manual quantification were determined from repeated measures of lesions in MS patient exams. Results indicate that assisted quantification reduced inter-operator variability from $0.34 \mathrm{~cm}^{3}$ to 0.17 $\mathrm{cm}^{3}$, and reduced intra-operator variability from $0.23 \mathrm{~cm}^{3}$ to 0.15 $\mathrm{cm}^{3}$. In addition, the minimum significant change between two successive measurements of lesion volume by the same operator, was $0.64 \mathrm{~cm}^{3}$ for manual quantification, and $0.42 \mathrm{~cm}^{3}$ for assisted quantification. For two different operators working successive measurements, the minimum significant change was $0.94 \mathrm{~cm}^{3}$ for manual quantification, but only $0.47^{3}$ for assisted quantification. Finally, assisted quantification reduced, by $1 / 2$ or more, the number of lesions which must be monitored for an average change in volume at a given power and significance level. These results suggest that assisted quantification may have practical applications in MS clinical trials.

\section{L-3}

\section{Amphetamine Failed to Improve Motor Function in Acute Stroke}

Rida Mazagri, A. Shuaib, M. McPherson and M. Deighton (Saskatoon, Saskatchewan)

There is convincing animal evidece that amphetamine improves outcome of function after brain injury. A limited study in patients (4 treated with amphetamine) showed promise that the drug may be effective in stroke patients.

We conducted a prospective stratified randomized and doubleblind study of the efficacy and safety of amphetamine in acute cerebral infarction in 25 patients. All these patients were admitted to the University Hospital with the diagnosis of cerebral infarction and motor weakness in the carotid or vertebro-basilar circulation. These patients were all adults and consents were obtained, received a single dose of amphetamine $(10 \mathrm{mg}$ ) or placebo orally within 72 hours from the start of the symptoms. Clinical evaluation was done one day before and 48 hours and 3 months after receiving the medications. Fugl-Meyer, Canadian neurological score and Barthel index were used in the clinical evaluation. The study did not alter the usual course of investigation and treatment of these patients. All patients had one hour of intense physiotherapy within hours after receiving the medications.

Recovery of motor function was not significantly different between the placebo and amphetamine treated groups. Our limited study indicates no benefit with amphetamine given within 24-48 hours after stroke.

\section{L-4}

Intracellular $\mathrm{Ca}^{2+}$ Chelator Ameliorates Synaptic Field Responses of Hippocampal CA1 Neurons Following Severe Forebrain Ischemia

S. Shinno, L. Zhang, M.C. Wallace and P. Carlen (Toronto, Ontario)

The mechanism of delayed neuronal death is not well understood, but the increase of intracellular $\mathrm{Ca}^{2+}$ during ischemia may play a critical role. Previous reports state that synaptic field responses are greatly reduced 2 - 3 days after global ischemia. This study was designed to verify the effect of pretreatment of BAPTAAM on the synaptic field responses after global ischemia. Adult male Wistar rats were exposed to $15 \mathrm{~min}$ of forebrain ischemia using a modified four-vessel occlusion (4VO) model. A single dose of BAPTA-AM $(20 \mathrm{mg} / \mathrm{kg})$ was slowly injected intravenously $1 \mathrm{~h}$ before $4 \mathrm{VO}$ in the treated group $(n=6)$. Non-treated rats were subjected to $4 \mathrm{VO}$ only $(\mathrm{n}=7)$. Synaptic field responses were recorded in CAl region $24 \mathrm{~h}$ after ischemia. Both amplitudes of somatic population spike (PS) and dendritic field EPSP were significantly improved in treated group as compared with non-treated group ( $\mathrm{p}<0.05$; PS: $3.1 \pm 1.7$ vs. $0.6 \pm 1.0[\mathrm{mV}$ ]; field EPSP: 1.8 \pm 0.5 vs. $0.4 \pm 0.4[\mathrm{mV}])$. Pair-pulse facilitation in dendritic field EPSP was also improved ( $p<0.05 ; 23.6 \pm 5.3$ vs. $7.8 \pm 13.4[\%])$. We conclude that BAPTA-AM is capable of preserving synaptic responses of CAl region at least $24 \mathrm{~h}$ after $15 \mathrm{~min}$ of forebrain ischemia. Increased intracellular $\mathrm{Ca}^{2+}$ may have a role in triggering delayed neuronal death.

\section{L-5}

\section{Brain SPECT Scanning with Tc-HMPAO in Acute Stroke}

Andrei V. Alexandrov, Lisa E. Ehrlich, Sandra E. Black and JOHN W. NORRIS (Toronto, Ontario)

Single-photon emission computed tomography (SPECT) is widely available in Canada and may have an important role in management of patients with acute stroke.

A total of 500 consecutive patients with acute stroke were studied. Clinical examination on admission (CNS) had the strongest predictive value for stroke outcome at two weeks $(\mathrm{p}<0.0001$, polychotomous logistic regression). SPECT had little additional value $(p<0.03)$ if performed anytime within the first week after stroke. Admission CT scanning had no prognostic value since it was false-negative in $48 \%$ of patients within the first 24 hours. SPECT performed better in patients in whom CNS failed to predict the outcome. Predictive value of cerebral perfusion imaging was the highest when SPECT was used within the "therapeutic window" in combination with TCD (Cerebral Perfusion Index): admission CNS, $p=0.03$; CPI, $p<0.0001$. SPECT identifies large volume ischemic lesions better than admission CT (absent perfusion pattern - $104 \pm 84 \mathrm{cc}$ ). Normal HMPAO distribution favors transient deficit or minor stroke, while HMPAO hyperfixation is associated with cardioembolic stroke. 
SPECT patterns of brain perfusion reliably predict the short-term outcome and lesion volume. SPECT is of particular value when admission clinical examination is inconclusive and $C T$ scan is negative. SPECT (and its combination with TCD) is suggested for stroke protocols and evaluating experimental therapies.

\section{L-6}

\section{Selection Criteria for Selective Peripheral Denervation (SPD) for Spasmodic Torticollis (ST)}

Reina Benabou, Pedro Molina-Negro and Guy Bouvier (Montreal, Quebec)

SPD has been a successful surgical treatment for patients with ST since 1987. It was developed as a result of experience from former techniques, anatomical and electrophysiological studies. It intends to abolish abnormal movements in all the muscles involved and preserve normal acting or inhibited muscles.

Over 350 patients had SPD for ST through these years. They were evaluated with a standard pre- and post-operative clinical and electrophysiological protocol. The follow up ranges from 1 to 16 years.

From these data we could establish the following premises as best indications for SPD: 1) The patient with ST should be refractory to previous conservative medical treatments and Botox injections, 2) The clinical picture should be stable for at least 1 year, preferably evolving for 3 years, 3) The dystonic symptomatology must be restricted to or, at least, prevalent in the cervical region, 4) Pure rotatory and its combinations with mild inclination and/or extension are the types of ST that obtained the best results $(88 \%$ excellent and very good), 5) Psychological features should be analyzed in individual basis.

Previous cervical surgical procedures, preexisting fibrosis and severe arthrosis have been shown to impair the post-operative results.

\section{L-7}

\section{Treatment of Laterocollis by Selective Peripheral Denervation}

Pedro Molina-Negro, Guy Bouvier and Reina Benabou (Montreal, Quebec)

Laterocollis or lateral inclination of the head is encountered as an isolated focal dystonia or, more frequently, associated with rotatory torticollis. Until recently we were under the impression that the ipsilateral trapezius combined with posterior muslces, were the main agonists of lateral tilting of the head. When denervation of posterior muscles fails to improve laterocollis, we verify the contribution of the trapezius by means of temporary bloc which usually results in marked reduction of movements of the shoulder. In most cases it was decided not to denervate the trapezius and some degree of laterocollis remained. Three years ago we started the analysis of the function of the levator scapulae (L.S.) with cadaver dissection, electrophysiological techniques and blocs. Our conclusion was that the L.S. is the main agonist of the lateral tilting of the head and consequently of laterocollis. We therefore define a surgical approach consisting in anterior ramisectomy combined with myectomy of the L.S. At the present time 20 cases of laterocollis have been treated with the new technique and the results are similar to those of the rotatory torticollis.

\section{L-8}

Foveal Colour Versus Motion Perception in Optic Neuritis,
Compressive Optic Neuropathy and Glaucoma

\section{J.J.S. BARTON and M. MUNSHI (Toronto, Ontario)}

It has been suggested that colour and motion are processed separately in parvocellular and magnocellular channels, and that optic neuritis and glaucoma affect channels selectively.

We studied foveal colour hue discrimination and motion direction discrimination in 12 patients with optic neuritis, 12 with optic nerve compression, and 17 with glaucoma. We used the Farnsworth-Munsell 100-hue test to study colour and random dot cinematograms to study motion perception.

Optic neuritis and optic nerve compression impaired hue discrimination more frequently and severely than motion discrimination. Glaucoma did not often affect either motion or colour perception. Motion perception was more often affected by optic neuritis than glaucoma, and was more often impaired in those with abnormal colour vision than in those with normal colour vision. Colour and motion scores correlated significantly with each other and with central luminance sensitivity on automated perimetry.

These results suggest that foveal luminance, colour, and motion perception may not be affected independently by these diseases. Either the diseases are not selective for subcortical channels at the fovea or moiton perception is not selectively processed in such channels.

\section{L-9}

\section{"Saccadic Jitter": a New Quantitative Ocular Sign of} Myasthenia Gravis

\section{J.J.S. BARTON and J.A. SharPe (Toronto, Ontario)}

Our purpose was to quantify the variability of saccadic peakvelocity/amplitude relationships in myasthenic and non-myasthenic ocular palsies. We measured centrifugal saccades in 9 patients with myasthenia gravis, 9 patients with proven non-myasthenic ocular palsies, and 3 normal subjects. Patients made repetitive saccades for 8 minutes. Saccades were analyzed at the start of the task, after 3 minutes of the task (fatigue), and 1 minute after edrophonium. We used a non-linear regression method to fit an exponential function to both individual saccades and saccadic averages for amplitude bins and calcualted the root mean square error of the curves. We subtracted the root mean square error of curves fitted to bin averages from that of curves fitted to individual saccades: the result was an index of the variability of saccadic peak velocity, which we called "saccadic jitter".

Compared to both normal subjects and non-myasthenic patients, the saccades of myasthenic patients showed more variability in both the initial and fatigue periods. The change induced by edrophonium did not distinguish between the groups. Signal detection analysis indicated that saccadic jitter has little value as a screening tool but is a diagnostic sign in $42 \%$ of myasthenic saccadic analyses. 


\section{Amantadine Hydrochloride Treatment in Heredo- Degenerative Ataxias: a Double-Blind Study}

M.I. Botez, Therese Botez-Marquard, Robert Elie, O.L. Pedraza, Kristine GoyetTe and Robert Lalonde (Montreal, Quebec)

A group of 27 patients with Friedreich's ataxia (FA) and another group of 30 subjects with olivopontocerebellar atrophies (OPCA) were each randomly divided into 2 subgroups, one receiving placebo and the other amantadine hydrochloride $(\mathrm{AH})(200 \mathrm{mg}$ daily) for $3 / 2-4$ months. The effect of double-blind treatment was evaluated by simple visual and auditory reaction time (RT) and movement time (MT) on both right and left hands. Therefore, for each patient there were $4 \mathrm{RT}$ and $4 \mathrm{MT}$ measures in milliseconds. The OPCA subgroup receiving AH showed significant improvement on all 8 parameters studied by analysis of covariance. Final data were adjusted taking into account the heterogeneity of the regression slopes. In FA patients, improvement was milder. The results suggest that $\mathrm{AH}$ should be administered to all OPCA patients as well as in some FA patients. Treatment remains contraindicated in those with cardiomyopathies and those with drug intolerance. The rationale of $\mathrm{AH}$ treatment in heredo-degenerative ataxias can be explained by 2 effects: i) dopamine release; and ii) the direct involvement of $\mathrm{N}$-methyl-D-aspartate (NMDA) in glutamate-mediated neurotoxicity in cerebellar granular cells. Memantine, an AH analog, is a potent blocker of NMDA receptors.

\section{L-11}

Idiopathic Cranial Pachymeningitis Presenting With Fluctuating Neurological Manifestations

M.A. BRyer, B.J. SchmidT and W.C. Halliday (Winnipeg, Manitoba)

A-46-year-old man presented with unremitting headaches in 1988; neurological and CT examinations were normal. In Dec 1989 he developed sizures. Biopsy of a left parietal white matter lesion, showed marked gliosis with mild glial atypia. By April 1991 his headaches subsided, anticonvulsant drugs were stopped, and no evidence of the parietal lesion was seen on CT or MRI. He remained well until Oct 1992, when he was reassessed because of headaches, a left NIV palsy, and an elevated ESR. Enhancement of the meninges, especially the falx and tentorium, was noted on CT and MRI; foci of increased MRI signal were present in the pons, cerebellum, and temporal lobes bilaterally. No granulomatous, infectious, or other disease was detected. A dural biopsy showed fibrosis with occasional perivascular lymphocytic cuffing. The left NIV palsy resolved. However, in May 1993 he developed headaches again and a right NVI palsy; the MRI showed worsening of meningeal enhancement but resolution of left temporal lobe edema. The right NVI deficit and headaches resolved over the course of several months, however, evidence of optic neuropathy appeared. Prednisone was started in April 1994. An MRI 5 months later showed marked improvement of dural enhancement and complete resolution of right temporal lobe edema.
The evanescent nature of this patient's manifestations and the response of the neuro-imaging abnormalities to steroid represent a novel presentation of this rarely described form of pachymeningitis.

\section{EPILEPSY (M-l to $M-1 I)$ \\ (Frida), June 23, 14:00 - 17:00)}

\section{M-1}

\section{Inheritance of Febrile Convulsions in Selected Large Families}

John MaHER and R.S. MCLaChLan (Winnipeg, Manitoba; London, Ontario)

Familial clustering of febrile convulsions is well recognized, twin and family studies supporting genetic influences. As part of a genetic linkage study in febrile convulsions, we explored modes of inheritance of febrile convulsions in families with at lease four members affected.

Of eight families, 75 members had febrile convulsions (26 males, 49 females). Vertical transmission was present through three to four generations, including from male to male. 51 and $46 \%$ of siblings and first-degree relatives of probands were affected. However, only 23 and $9 \%$ of second- and third-degree relatives were similarly affected.

An autosomal dominant mode of inheritance of febrile convulsions is suggested in these selected large families, based on the pattern of transmission and the proportion of affected relatives, although a polygenic basis with one predominant gene is another possibility. Why the risk falls over generations is unclear, but may be due to ascertainment biases or modifier genes.

\section{M-2}

\section{The Natural History of Late Febrile Seizures}

D.W.M. Webi, R. Jones, A. Manzur and K. Farrell. (Vancouver, British Columbia)

Benign febrile convulsions are an age specific epilepsy syndrome with favourable prognosis. The natural history of seizures occurring with fever after the age of five years is less clearly defined.

We reviewed the Neurology records at our hospital and identified 33 children who had seizures only associated with fever after the age of five years. Outcome was assessed by chart review and parental interview.

A history of febrile seizures befor the age of five years (14 male, 11 female) was noted in 25 . In this group, seizures with fever continued beyond seven years in 12 and nine years in four. Four (including one child with learning difficulties) developed afebrile seizures.

Eight children had their first seizure with fever after the age of five (six male, two female). Seizures with fever recurred in six patients. Two of this group have a first degree relative with febrile seizures. All have normal development and none have had afebrile seizures. 
Children with febrile seizures may continue to have seizures with fever after the age of five. This may be associated with a modestly increased risk of afebrile seizures. Children presenting with their first seizure associated with fever after the age of five often have a favourable outcome.

\section{M-3}

The Modulating Effect of Gender and Age of Seizure Onset on Intellectual Outcome in Children With Partial Seizures

K.A. Selby, K. Farrell, E.H. Strauss, J. Zaide and M. HUNTER (Vancouver, Victoria, British Columbia)

In a retrospective study of children with partial seizure disorders, we examined the influence of gender and age at seizure onset on cognitive outcome.

We identified 40 children with definite lateralization of siezure focus, based on clinical, neurophysiological and neuroradiological assessment. Comprehensive neuropsychological evaluation was performed on all children.

Age at seizure onset was an important predictor of intellectual outcome $(p<0.03)$. The average full scale IQ was 76 in those with seizure onset before 12 months and was 94 in those with seizure onset after 12 months. The effect of age of onset of seizures on intellectual outcome was observed only in males ( $p$ $<0.05$ ). Age of onset of seizures had no influence on intellectual outcome in females.

These data would be consistent with an influence of gender on the seizure threshold in the young child or on the effect of seizures on the developing brain. These may relate to gender differences in the rate of brain maturation or to the influence of sex hormones.

\section{M.4}

\section{Predicting Exacerbation of Seizures and Response to Vigabatrin and Lamotrigine}

\section{H.Z. DARWISH and V. LANGE (Calgary, Alberta)}

It is often quoted that failure of response to one or two major anti-epileptic agents implies a refractory state to medical therapy.

We report our experience with 39 children with a mean age of $8.9 \pm 5$ years, whose partial complex and secondary generalized seizures had failed to three or more medications.

15 were of normal intelligence and had no abnormalities on exam or neuro-imaging. 24 had abnormality on imaging $(\mathrm{N}=$ 16) and/or a mental handicap $(N=24)$. These children all received Vigabatrin add-on trial and when that failed, some subsequently went on to a Lamotrigine trial $(\mathrm{N}=15)$.

$12 / 39$ children had $>50 \%$ reduction in seizure frequency on Vigabatrin. In 9/39 children Vigabatrin induced a dramatic increase in seizure frequency. Seven of these nine children were mentally normal whereas only $2 / 12$ responding to Vigabatrin were normal. The age of those with seizure exacerbation was 5 \pm 4 years. The interval to seizure exacerbation was $0.53 \pm 0.6$ months and the dose was $56 \pm 29 \mathrm{mgm} / \mathrm{kg}$ whereas in those responding to Vigabatrin it was $48.6 \pm 13.2 \mathrm{mgs} / \mathrm{kg}$.

$15 / 27$ Vigabatrin failures were treated with Lamotrigine.
$9 / 15$ had a $>50 \%$ seizure reduction with a dose $8 \pm 9 \mathrm{mgm} / \mathrm{kg}$. $5 / 9$ had no mental handicap.

We conclude that newer anti-epileptic medication can be effective in children with otherwise refractory partial complex and secondary generalized epilepsy (53\%). Normal mental ability and younger age suggested a group of children at greater risk for Vigabatrin induced exacerbation of seizures.

\section{M-5}

A One-year Follow-up Study in Adults With Uncontrolled Complex Partial Seizures on Vigabatrin (Sabril@): The Canadian Experience

A. Guberman, J. Bruni, C. Desforges, M. Jones, M.-A. Lee, R. Mclachlan, A. Ogunyemi, N. Pillay, M. Sadler, E. Starreveld, N. Sussman, L. VaChon and D. Weaver (Ottawa, London, Kingston, Ontario; Laval, Quebec; Vancouver, British Columbia; Calgary, Edmonton, Alberta; St. John's, Newfoundland; Winnipeg, Manitoba; Halifax, Nova Scotia; Kansas City, Missouri)

Treatment with vigabatrin ( $\gamma$-vinyl-GABA, Sabrilß) was followed up in open-labelled conditions for one year, in patients (age $19-51$ ) with epilepsy who had previously participated in a 36-week double-blind, placebo-controlled, multicentre study (Guberman et al., Can J Neurol Sc 21 [Suppl. 2]: S17 - 18, 1994). Out of 97 patients enrolled, 53 (55\%) completed the trial, on 2 - 4 grams per day. At the end of treatment, the baseline median monthly frequency of complex partial seizures (with or without secondary generalization) had decreased from 8.6 (95\% C.I. $=6.5-10.5)$ to $4.0(2.5-6.0)$ in the intent-to-treat population, and from $7.0(6.0-9.0)$ to $1.5(1.0-2.5)$ in patients who completed the study. A decrease in seizure frequency $\geq 50 \%$ was observed in $54 \%$ of the patients, including $11 \%$ who were seizure free at the end of the study. Lack of benefit (27\%) and adverse events $(13 \%)$ represented the main reasons for premature discontinuation. Adverse events most frequently reported (11 - 26\%) included fatigue, headache, dizziness memory disturbances, agitation, nystagmus and drowsiness. Safety assessments did not show major, clinically significant changes. These results demonstrate that more than $50 \%$ of patients with uncontrolled seizures display a sustained benefit following $12-20$ months of treatment with vigabatrin used as add-on to a current antiepilepsy medication regimen.

\section{M-6}

Pathology and Imaging in Pediatric Temporal Lobectomy for Intractable Epilepsy

D.B. Sinclair, J. McKean, K. Aronyk, M. Javidan, M. HoSkINGSON and B. Mielke (Edmonton, Alberta)

We reviewed the pediatric cases of temporal lobectomy performed for intractable partial complex seizures at the University of Alberta Hosptial. 26 cases are reviewed, and the radiological and pathological features described. The patients range from 18 months to 16 years old. The most common pathology was mesial temporal sclerosis or temporal lobe gliosis (MTS/TLG) 
which was found in 12/26 patients. In six patients dual pathology was seen (MTS and developmental abnormalities). Six patients had tumours, one a porencephalic cyst, and one the form fruste of tuberous sclerosis. The CT Scan was abnormal in $66 \%(4 / 6)$ of tumour patients but abnormal in only $33 \%$ of the patients (4/12) with MTS/TLG or dual pathology (2/6). The MRI was accurate in all cases of tumour and most cases of dual pathology (5/6) but was abnormal in only 50\% (6/12) of MTS/TLG patients. Both modalities accurately detected the patients with porencephalic cysts and tuberous sclerosis. Overall, 77\% (20/26) of the patients were seizure free following surgery with the follow up ranging from one to seven years (mean 3.6 years). $83 \%$ of both the tumour and the dual pathology patients were seizure free while $88 \%$ of the patients with MTS were seizure free in follow up.

\section{M-7}

\section{A Prospective, Non-randomized, Controlled Trial of Seizure Outcome Following Temporal Lobectomy}

R.S. Mclachlan, C. Bonnar, P. Derry, W.T. Blume and J. Girvin (London, Ontario)

Background: Numerous retrospective surveys of seizure outcome following epilepsy surgery have been done but this is the first study in which outcome is compared prospectively to that with medical management. Methods: 70 consecutive surgical candidates with epilepsy were investigated in our epilepsy unit. Two groups of patients, those who underwent temporal lobectomy and those who continued medical management without surgery were followed up at 6,12 and 24 months after discharge from hospital. Results: Temporal lobectomy was done in 52 patients (28 left, 24 right) and 18 had no surgery (no focus found, risk too high, refused). Pathology was mesial temporal sclerosis $(62 \%)$, tumour $(8 \%)$, other (30\%). At 12 months, outcome of surgical vs. medical management was 1) completely seizure free, $40 \%$ vs. $0 \%, 2$ ) simple partial seizures only, $13 \%$ vs. $0 \%, 3$ ) rare seizures, $13 \%$ vs $0 \%, 4$ ) worthwhile improvement, $21 \%$ vs. $6 \%, 5$ ) some improvement, $6 \%$ vs $28 \%, 6$ ) no improvement, $6 \%$ vs. $66 \%$. Mean duration since last seizure in categories $3,4,5$ and 6 was $6,2,<1,<1$ months. A satisfactory outcome (categories $1-4$ ) occurred in $88 \%$ and $6 \%$ respectively $(\mathrm{p}<.0001)$. Satisfactory outcome at six months was $91 \%$ vs. $14 \%(\mathrm{p}<.0001)$ and at 24 months, $93 \%$ vs. $0 \%$ ) p <.0001). Conclusion: 1) Surgical treatment of intractable temporal lobe epilepsy results in better seizure control than medical management. 2) Improvement is sustained over two years.

\section{M-8}

Microglial and Astroglial Response in Hippocampal Sclerosis Associated With Temporal Lobe Epilepsy: Evidence of Progressive Neuronal Loss

T.G. BEACH, W.B. WoOdhuRst and M.W. Jones (Vancouver, British Columbia)

Hippocampal sclerosis (HS) is the most common pathological finding in temporal lobe epilepsy. HS has classically been regarded as inert scar tissue resulting from a destructive event in the remote past. Conversely, modern theories of epileptogenesis, based on experimental work done in animals, propose that ongoing seizure activity causes continuing neuronal degeneration by an excitotoxic mechanism. The hippocampi from 22 patients with HS as the sole significant pathological diagnosis after temporal lobe resection were compared to those from nine agematched autopsy cases with no history of neurological disease. Immunohistochemical methods were used to localize GFAP, an astrocyte-specific intermediate filament, and HLA-DR, an MHC class II membrane surface receptor, in order to reveal the presence of reactive astrocytes and microglia, respectively. Cases of HS had markedly increased numbers of both cell types, compared to autopsy controls. Experimentsl studies in animals have shown that activated microglia are seen only in the subacute period after a destructive lesion. Astrocytic hypertrophy can persist for years after a destructive lesion, but the presence of multinucleated astrocytes, which were seen frequently in the dentate gyrus molecular layer of HS cases, is strongly suggestive of active astrocytic proliferation. These results support the existence of progressive neuronal loss in HS associated with temporal lobe epilepsy.

\section{M-9}

Mesial Temporal Sclerosis 1995. Still an Underdiagnosed and Undertreated Neurosurgical Disorder

Michael W. Jones, B. Woodhurst, D. MacDonald, T. Beach and J.WADA (Vancouver, British Columbia)

We analyzed our last 40 cases of pathologically confirmed Mesial Temporal Sclerosis (MTS) who had greater than one year follow-up. All had an ECoG tailored anterior temporal lobectomy for drug refractory epilepsy. Only $25 \%$ of these patients were referred for surgery by neurologists!

This is a stereotyped clinical syndrome that if recognized should lead to a surgical referral if standard AEDs don't work. Results: $58 \%$ were women; $47 \%$ had Lt. sided focus. In $53 \%$ of cases there was a history of febrile seizures; $78 \%$ had MTS on their MRI. All had Complex Partial Seizures and only $45 \%$ had associated second Gen. Tonic-Clonic Seizures. The mean age of habitual onset was seven years, mean duration of epilepsy was 21 years and mean age at surgery was 28 years. Engel's classification for follow-up; $78 \%$ were Class 1 (no seizures); $18 \%$ were Class 2 (rare seizures) and $4 \%$ were Class 3 (definite improvement). Conclusion: Surgery cured $76 \%$ and markedly improved another $18 \%$ for a collected $96 \%$ overall improvement.

\section{M-10}

Surgical Treatment of Epilepsy and Non-Neoplastic Lesions of the Temporal Lobe

W.B. WOODhuRST, D.B. MacDonald, M.W. Jones, J.A. WADA and K. BERRY (Vancouver, British Columbia)

Nine patients with non-neoplastic lesional temporal lobe epilepsy had resections 1988 to January 1994. These are from a 
consecutive series of 92 operations including 21 other structural neoplastic lesions to which they will be compared.

Seven lesions were cavernous angiomas, three dominant hemisphere, four non-dominant hemisphere, one lesion was AVM, non-dominant, and one a cysticercus cyst, dominant.

Electrocorticography $(\mathrm{ECoG})$ varied from well localizing congruent with gross and microscopic pathology (4/9) to poorly congruent ECoG. Poor congruence was primarily mesial subtemporal spikes with laterally located pathology.

Resections included all the symptomatic lesion(s) and with few exceptions all the ECoG abnormalities.

Two pts with resection of ECoG abnormal anatomically normal tissue of dominant anterior mesial structure had mild nondisabling worsening of memory, one subjective only, one transient objective only. One patient with similar ECoG abnormalities of mild degree had sparing of hippocampus with no cognitive change subjectively.

All pts had Engel Class I outcome. All pts improved upon their previous level of psychosocial function.

The ECoG is of value in these procedures when considered in the context of lesion anatomy and neuropsychological variables.

\section{M-11}

\section{Mesial Occipital Epilepsy: Electroclinical Features and Surgical Outcome}

Samuel, Wiebe, Lisa Marie Oliver, Warren T. Blume and JOHN P. GIRVIN (London, Ontario)

Objectives: To identify electroclinical features of mesial occipital seizures and to comprehensively assess outcome of surgical resection. Methods: Review of patients with electrocorticographically proven, medically refractory mesial occipital seizures. Prospective assessment of quality of life, seizure severity, and clinical status. Results: 11 patients were investigated and operated on. Median age and age of onset: 24 years (16 $41)$ and 10 years $(3-34)$, respectively. All patients seizures started with predominantly contralateral elementary visual phenomena. $10(91 \%)$ had complex partial and/or generalized motor seizures. Only one had exclusively simple partial visual seizures. Scalp EEG: Epileptiform activity occurred in eight patients. This was exclusively occipital in only one subject. All others had broad posterior head involvement. Two exhibited light sensitivity. Electrocorticography: All had mesial occipital recorded seizures. Exclusively occipital eight (73\%), plus extraoccipital three. Imaging, Neuropathological and cortical stimulation findings were reviewed. Median follow-up (available in 10), was two years ( $1-8$ years). Four were seizure-free, three were significantly improved. Five had complete and two incomplete homonymous hemianopsia, two had quadrantanopsia, and two central-paracentral scotomata. Ten $(91 \%)$ are currently employed and/or full-time students. Patient-centred, health-related quality of life was determined in eight subjects using seven point Likert scale global ratings. Clinically significant improvement occurred in six $(75 \%)$. Perceived working/studying ability improved in three.

\section{N. NEUROSURGERY - VASCULAR ( $\mathrm{N}-\mathrm{l}$ to $\mathrm{N}$ - 11 ) (Friday, June 23, 14:00 - 17:00)}

\section{N-1}

The Immediate Histopathology of Cerebral Vasospasm and Reversal of Vasospasm After Effective Cerebral Angioplasty: a Case Report

P.A. Pahapill, J. Siddiei, D.A. Ramsay, A. Fox, L. Assis and H.W.K. BARR (London, Ontario)

Cerebral vasospasm is a major source of morbidity and mortality in patients with subarachnoid haemorrhage secondary to a ruptured intracranial aneurysm. The beneficial role of cerebral angioplasty in the treatment of vasospasm is well known but its mechanism remains unclear. The risks of cerebral angioplasty are rare but do include potential rupture of treated vessels. We report a case of intracranial arterial rupture during effective cerebral angioplasty in a patient with cerebral vasospasm secondary to spontaneous subarachnoid haemorrhage. The immediate histopathology of active cerebral vasospasm and subsequent effective reversal of vasospasm by cerebral angioplasty in this patient is presented.

\section{N-2}

Double-blinded, Placebo-controlled Efficacy Study of Intracisternal Recombinant Tissue Plasminogen Activator (rt-PA) in Patients with Aneurysmal Subarachnoid Hemorrhage

J.M. Findlay, N.F. Kassell, Gail Kongable and B.K.A. Weir (Edmonton, Alberta; Charlottesville, Virginia; Chicago, Illinois)

The purpose of the study was to determine the safety of intrathecal $\mathrm{rt}-\mathrm{PA}$ and its effect on angiographic vasospasm. 100 patients, 18 years or older with aneurysmal SAH received a single intraoperative intracisternal instillation of $10 \mathrm{mg} \mathrm{rt}-\mathrm{PA}$ (activase R) or placebo following aneurysmal repair. Surgery was performed \pm 72 hours post-SAH, and all patients received Nimodipine. Hypertensive/hypervolemic therapy, angioplasty, and therapy for SAH complications were determined by the treating investigator.

Forty-three patients received placebo, and 48 received rt-PA. Nine randomized patients did not receive treatment following complicated surgical procedures. Differences in factors prognostic for poor outcome were not statistically different in the two groups: age $p=0.62$, admission GCS $p=0.26$, clot thickness on CT $p=$ 0.88 , and admission hypertension $p=0.97$. The overall incidence of angiographic vasospasm was not significantly different in the two groups $(p=0.31)$, however severity of vasospasm (moderate or severe) was found to be less severe in the rt-PA treated group (p $=0.07$ ) and was less common and severe in those patients with thick clots on CT $(p=0.02)$. Intracranial bleeding complications occurred in 6 placebo treated patients ( 1 severe) and 9 rt-PA treated patients ( 2 severe), $p=0.54$. Causes of diability and death were not statistically different in the two groups. Overall outcome based on the Glasgow Outcome Scale was not statistically different at 3 months $(p=0.92)$ in this small series of patients. Conclusions are limited by a small sample size, however this study suggests that intracisternal rt-PA affords acceptable risk and could benefit patients at high risk for vasospasm. 
Intracranial Carotid Occlusion During Carotid Thromboendarterectomy: Reducing the Risk of Intraluminal Thrombus?

J.M. FINDLAY and M.C. WALLACE (Edmonton, Alberta; Toronto, Ontario)

Large, floating, intracarotid thrombi on cerebral angiography identify patients at high risk of stroke. The significant risk of surgical manipulation dislodging emboli into the cerebral circulation and causing intraoperative stroke has led to the suggestion that patients with intraluminal thrombi be treated with anticoagulants at least until the thrombus spontaneously resolves on follow-up angiography. Recent data from NASCET, however, suggests that treatment with anticoagulation or thromboendarterectomy are equally dangerous.

Preceding middle cerebral embolectomies in 2 patients with severe internal carotid artery (ICA) stenoses with attached intraluminal thrombi allowed intracranial access to temporarily occlude the supraclinoid ICA during the critical stage of thromboendarterectomy (as part of a combined embolectomy/endarterectomy procedure).

We have since performed craniotomies solely for supraclinoid ICA occlusion during cervical ICA thromboendarterectomy in 3 additional patients, with large and high propagating intraluminal thrombi, with good results.

Temporary supraclinoid ICA occlusion may increase the safety of carotid thromboendarterectomy in patients with large, floating intraluminal thrombi at high risk of stroke with standard treatments. It can be considered along with anticoagulation.

\section{N-4}

\section{Carotid Surgery Results in Postgraduate Training Program}

M.C. Wallace, G. Leung, R. Khan, F. Gentili and R. Fleming (Toronto, Ontario)

The expanding role of carotid endarterectomy in stroke prevention requires defined perioperative morbidity and mortality. This mandates a thorough and hands-on surgical experience at the postgraduate level to prepare surgeons-in-training for practice. This study explores a prospective series of patients operated on at a teaching institution.

Most patients were symptomatic (187/190). A resident or fellow was the primary surgeon in 168 cases $(88 \%)$. The resident and fellow were primary surgeon and first assistant in 99 cases (52\%). Six strokes occurred $(3.2 \%)$, with deficit at 6 months present in only one patient $(0.5 \%)$. One patient had a myocardial infarction $(0.5 \%)$ and one patient died $(0.5 \%)$. The hematoma rate was $17 \%$ (33/190) with reintubation or operation required in 14 patients $(7 \%)$. There was no statistical difference in morbidity (stroke, hematoma) in staff or trainee operated cases.

The delegation of surgical responsibility to postgraduate trainees in neurosurgery is safe, maintaining excellent results for effective stroke prevention.

\section{Internal Carotid Artery Dissection: Management Chaos?}

J. SIDDIQI, M.-G. BOUSSER and V. HACHINSKI (London, Ontario; Paris, France)

The first case of "spontaneous" extracranial internal carotid artery dissection (SEICAD) was reported in 1954. Increasing awareness of SEICAD has overturned original conceptions of the condition: from a rare disease of which only 10 cases were reported in the 1954-1974 period, to a condition of which over six hundred cases are present in the English and French language literatures; from a truly "spontaneous" condition to one in which micro-trauma or macro-trauma is implicated; from a highly malignant condition presenting always with stroke (from which eight of the first ten reported cases succumbed), to a relatively benign disease with protean manifestations and a 10 year survival rate of $80 \%$; and, from primarily a surgical disease, to primarily a medical one. Much of this reversal has been based on empiricism, not on systematic study. Indeed, for a condition so frequently reported in the literature, the knowledge gaps regarding epidemiology, pathogenesis, natural history, and management are enormous. We illustrate the chaos that persists in a proper understanding and management of SEICAD, and propose that the time is ripe for a randomized therapeutic trial in Canada.

\section{N-6}

\section{Carotid Endarterectomies With Routine Use of Shunt Device;} Results in $\mathbf{5 0 0}$ Consecutive Cases

Eric Bélanger, Daniel Lacerte, Jean-Françols Turcotte and JEAN-MARIE BouCHARD (Quebec, Quebec)

The controversies about the type of anesthesia, the optimal monitoring device and the use or not of a shunt in performing carotid endarterectomy remain unresolved. However, majority of recent reports showed downward trend for CEAs complication performed with any well recognized technique. The purpose of this retrospective study was to analyze the mortality and morbidity associated with general anesthesia, routine use of a shunt without cerebral monitoring.

The records of 500 consecutive CEAs, performed over the last 10 years, were analyzed with care, and the literature used for comparison. We found a combined mortality-morbidity of $3 \%$. The incidence of permanent disability from stroke was $1 \%$ and five patients (1\%) died.

Our results suggest that performing CEAs under general anesthesia with routine use of a shunt can be safe and efficient. The complication rate can be compared to other large series using different surgical approaches. We also discuss rational basis supporting our approach. 
N-7

Role of Embolization in the Management of Brain AVM Patients Presenting With Progressive Neurological Deficit

K.G. Terbrugge, R.A. Willinsky, W.J. Montanera, M.C. WaLLACE and E. HaTSWELL (Toronto, Ontario)

In order to assess the role of endovascular treatment of patients with brain arteriovenous malformations (AVMs) presenting with progressive neurological deficit we reviewed our experience at The Toronto Hospital.

Between 1984 and 1994, 546 patients with brain AVMs were referred to our institution for consultation and possible treatment. Twenty patients presented with progressive neurological deficit (P.N.D.) of which 8 patients were selected for embolization. Follow-up after embolization ranged from three months to nine years. Seven patients either stabilized or improved after partial embolization and one worsened. Our experience indicates that embolization should be considered in the management of brain AVM patients presenting with P.N.D.

Detailed angiographic analysis has helped us to select those patients with P.N.D. whose formidable AVMs might benefit from partial embolization.

\section{$\mathbf{N}-8$}

\section{An Analysis of Complications from Endovascular Treatment} of Brain Arteriovenous Malformations

Peter B. Dirks, Michael Tymianski, Robert Willinsky, Walter Montanera, M. Christopher Wallace and Karl TerBrugge (Toronto, Ontario)

Endovascular embolization of brain arteriovenous malformations (AVMs) has become an important component of a multimodality treatment approach to these lesions. To date, little has been published concerning complications of this procedure. We have reviewed all patients undergoing endovascular treatment with liquid adhesive agents for AVMs at our institution between 1985 and January 1995 to analyse the complications of this procedure. 546 brain AVM patients have been assessed for therapy. 209 patients underwent 387 embolization procedures. Complications were subdivided into hemorrhagic and non-hemorrhagic events and were classified into immediate ( $<24$ hours), early ( $<30$ days), and late ( $>30$ days). 45 events occurred following 387 procedures (Table 1).

\section{Table 1.}

\begin{tabular}{lccc}
\hline & $\begin{array}{c}\text { Hemorrhage } \\
\text { (total \# patients/\# }\end{array}$ & $\begin{array}{c}\text { Ischemia } \\
\text { permanent }\end{array}$ & $\begin{array}{c}\text { Other } \\
\text { disability or dead) }\end{array}$ \\
\hline Immediate & $7 / 6$ & $16 / 4$ & $7 / 1$ \\
Early & $3 / 3$ & $0 / 0$ & $0 / 0$ \\
Late & $11 / 5$ & $1 / 0$ & $1 / 0$ \\
\hline
\end{tabular}

The frequency of permanent disability or death in the 30 days after an endovascular treatment is $3.6 \%$. Most events with severe neurologic sequelae occurred due to an immediate hemorrhage. Ischemic events were usually immediate but transient (recovery within 24 hours). Most "other" immediate complications related to a retained catheter within the cerebral vessels, without sequelae. 11 patients had a delayed hemorrhage from their AVM after partial endovascular obliteration. It is unclear whether these hemorrhages were a result of endovascular treatment or are due to the natural history of the disease. We are now trying to identify factors which may be predictive of a hemorrhagic complication following endovascular treatment.

\section{N-9}

Prediction of Obliteration of Arteriovenous Malformations After Radiosurgery: The Obliteration Prediction Index

\section{M.L. Schwartz, K. Sixel, C. Young and P. O'Brien (Toronto, Ontario)}

It has been observed that the obliteration of brain arteriovenous malformations (AVMs) after radiosurgery depends upon the size of the AVM and the radiation dose administered to the margin of the lesion. In our centre, of 91 patients treated for AVMs, 44 have so far been imaged at two years or longer after treatment. Setting aside six apparently obliterated AVMs with MRI follow-up only, (as MRI is less sensitive in the detection of AVM remnants than angiography), and five cases where an early-filling draining vein was visible, the "obliteration prediction index" of 33 cases was calculated by dividing the marginal dose of radiation in Grey (Gy) by the lesion diameter in centimetres. Those AVMs that were obliterated had a mean index of 10.6 , whereas those that failed to disappear completely had a mean index of 7.6. These populations are statistically distinct $(\mathrm{P}=0.0297)$. This dichotomy permits prediction of the probability of successful outcome for individuals undergoing radiosurgical treatment on the basis of the index derived from these two attributes.

\section{N-10}

\section{The Effect of Mannitol on Experimental Cerebral Ischemia -} Revisited

Terri L.C. Luvisotto, Roland N. Auer and Garnette R. SUTHERLAND (Calgary, Alberta)

The effect of mannitol on ischemic neuronal injury was examined in male Wistar rats $(\mathrm{n}=80)$ randomly divided into either forebrain or focal ischemia groups. The animals were further subdivided into control, mannitol treatment preischemia, and mannitol treatment post-ischemia groups. Physiologic parameters were monitored and maintained within normal limits. Mannitol administration post-ischemia was found to produce a statistically significant improvement in ischemic neocortical injury and selective neuronal necrosis in the neocortex in the forebrain and focal models respectively. However, post-ischemia mannitol treatment was not shown to favourably alter histopathologic outcome in hippocampal levels 2 through 7 in the forebrain model, nor did it reduce infarct volume in the focal ischemia group. Pre-ischemia treatment with mannitol did not produce statistically significant reductions in neuronal injury in either model. These results do not support the findings of previous investigations which have demonstrated that mannitol affords significant neuroprotection when administered either before or immediately following vessel occlusion in models of forebrain and focal cerebral ischemia. Mannitol's ability to reduce ischemic neuronal injury when administered following ischemia is consistent with a beneficial influence on the development of delayed cerebral edema. 


\section{N-11}

\section{The Effect of Age in Models of Cerebral Ischemia}

Gary A. Dix, Roland Auer and Garnette R. Sutherland (Calgary, Alberta)

Models of global and focal cerebral ischemia are associated with a variety of metabolic perturbation that, if uncorrected, progress to irreversible brain damage. Although a number of variables have been shown to affect the severity of neuronal injury, the influence of age has not been well-established.

In this study 51 Wistar rats, both young (2 - 4 months) and old (20 - 24 months), were subjected to either focal or forebrain ischemia. The animals were sacrificed on day seven post-ischemia and the distribution and extent of brain damage was quantified. In the focal ischemia group, old rats had significantly greater striatal damage $(P<0.01)$ as well as an increase in penumbral ischemia neurons. In contrast, the global model showed statistically significant increases in hippocampal neuronal injury in young rats compared to old rats $(\mathrm{P}<0.003)$. Within the striatum, however, neuronal damage was significantly worse in the old rats.

These data further reiterate the importance of age as a variable in models of cerebral ischemia. The unique observation in this study is that the response to age differed in global and focal models. This dichotomy supports the growing consensus that the pathophysiology of brain injury differs between these two main experimental paradigms.

\section{O. NEUROSURGERY - PEDIATRIC $(O-1$ to $0-11)$}

(Friday, June 23, 14:00 - 17:00)

\section{0-1}

\section{Scalp Tissue Expanders for Closure of Large Cutis Aplasia} Congenita

Wendy Zial, D. McPhalen, S.T. Myles and M.G. Hamilton (Calgary, Alberta)

Scalp cutis aplasia congenita represents a heterogeneous group of disorders characterized by focal absence of the scalp which may be associated with defects of the periosteum, skull or dura. While small defects frequently heal satisfactorily without surgical treatment, larger lesions with osseous defects are at risk for infection, sagittal sinus thrombosis, and hemorrhage. Early surgical scalp reconstruction has been advocated for children with large cutis aplasia congenita to decrease the risk for these potentially lethal complications. A number of different surgical strategies have been proposed to provide initial coverage of the scalp defect, including split thickness skin grafts or early rotational scalp flaps. However, the treatment of large cutis aplasia defects with either of these strategies in the neonate may be complicated by: 1) the inability to provide adequate coverage of the defect or problems with survival of rotational flaps, and 2) the inability of split thickness skin grafts to provide protection from hemorrhagic complications.

Three children with scalp defects greater than $10 \mathrm{~cm}$ diameter have been treated at the Alberta Children's Hospital. The first two children in the series were managed utilizing standard techniques with unsatisfactory full thickness scalp coverage. We have recently utilized scalp tissue expanders to aid in the treatment of a neonate with a large cutis aplasia scalp defect. Scalp tissue expanders provided the opportunity to increase both the size and the vascularity of the scalp flaps prior to rotation. The authors recommend that scalp tissue expanders be considered during the initial surgical management of children with large cutis aplasia congenita.

\section{0-2}

Appendicitis in Children With Myelomeningocele and a Ventriculoperitoneal Shunt

WENDY Ziai, S.T. Myles and M.G. Hamilton (Calgary, Alberta)

Appendicitis in children or adults with myelomeningocele has a substantial morbidity when compared to the general population. This may be due in part to a higher incidence of appendicial rupture in these patients. Furthermore, the issues concerning management of the shunt system in the presence of acute appendicitis and appendicial rupture are important.

The records of the Alberta Children's Hospital were reviewed for a five year period (1989 - 1994) searching for all children with the diagnosis of myelomeningocele who had been treated for acute appendicitis. Of the five children identified, four had a ventriculoperitoneal (VP) shunt. All four of the children with VP shunts had experienced appendicial rutpure, however, all patients had sterile CSF at the time of presentation. The last two patients in this series represent the basis for the following neurosurgical management strategy which can be successfully applied when children or adults with VP shunts present with peritonitis.

A patient with a VP shunt presenting with acute appendicitis undergoes simultaneous externalization of the peritoneal catheter (with CSF sampling) and appendectomy. As soon as the patient is clinically stable, the VP shunt is converted to a ventriculo-atrial (VA) shunt (if the CSF is sterile), thereby avoiding the infected, inflamed peritoneal cavity. The atrial catheter is percutaneously placed via the Seldinger technique into the internal jugular vein using a 12-French peel-away sheath. Early reinternalization of CSF flow via a VA shunt significantly reduces the risk of CSF infection and shortens hospital stay by allowing the patient to be discharged home as soon as they have recovered from their appendicitis.

\section{$0-3$}

Comparison of Syringopleural and Syringo-subarachnoid Shunting in the Treatment of Syringomyelia in Children

Jean-Pierre Farmer, Olivier Vernet and José L. Montes (Montreal, Quebec; Lausanne, Switzerland)

Case records with the diagnosis of shunted syrinx were retrospectively reviewed. om 1984 - 1994, 31 patients had their syrin $x$ treated by syringopleural (19 cases/ Gr. A) or syringosubarachnoid (13 cases/Gr. B) shunting. One patient was included in both groups. Associated diagnoses were: In Gr. A-2 Chiari 1 and 14 Chiari 2 malformations, 14 shunted hydrocephalus, 13 spina bifida aperta and three occulta; in Gr. B-5 Chiari 1 and 2 Chiari 2 malformations, three shunted hydrocephalus, two spina bifida aperta and five occulta. Eight Gr. A and six Gr. B patients had undergone prior Chiari decompression. Motor deficits predominated in both groups. Pachymeningitis was a uniform operative finding. Neurological follow-up shows $11 \mathrm{Gr}$. A patients 
improved and eight stabilized whereas on MRI eight cavities collapsed and 10 were markedly reduced. One patient was reoperated for pleural effusions and one for shunt displacement. In Gr. B one patient improved, eight stabilized, three worsened, and one was lost to follow-up. Radiologically, one cavity collapsed, six reduced significantly, two were unchanged, and three enlarged. Four patients were offered reoperation for neurological and/or radiological deterioration, one refused. We conclude that: a) SP shunting is superior to SSA shunting; b) Chiari decompression is suboptimal at controlling it; c) perimedullary pachymeningitis may be an important etiological factor; and d) SP shunting is a viable option for controlling syringomyelia in patients otherwise asymptomatic from their Chiari 2 malformation.

\section{$0-4$}

\section{Primary "Malignant Rhabdoid Tumour" (MRT) of the Central Nervous System}

J. Siddiqi, D.A. Ramsay, R.F. Del Maestro, B. Cairney, B. FISHER and D.R. MACDONAND (London, Ontario)

MRTs are rare, enigmatic, paediatric renal neoplasms. Exceptionally, MRTs, or "quasi" MRTs, can also occur in other sites (Parham DM, Weeks DA, Beckwith JB, 1994, Am J Surg Pathol 18: 1010-1029), including the brain where their prognosis is worse than that of a glioblastoma, owing to their resistance to chemotherapy and radiotherapy. Two cases of primary cerebral MRTs are described, located in the occipital lobes of two girls, ages six and nine years. In both cases, the tumours were well-circumscribed, and unevenly enhancing on CT scan of the head. In both patients, there was no radiological evidence of a primary extraneural source, and malignant cells were present in the lumbar cerebrospinal fluid. Histologically, many of the tumour cells showed eccentric, large eosinophilic, vimentinimmunolabellable, cytoplasmic inclusions and no ultrastructural (younger case) or immunohistochemical evidence (both cases) to support a glial (glial fibrillary acidic protein) or neural (synaptophysin, neurofilaments) origin. The tumours were resistant to radiotherapy and chemotherapy, and both patients died within three months after presentation. The putative relationship between these highly malignant brain tumours and the classical renal MRTs will be explored.

\section{0-5}

Pleomorphic Xanthoastrocytoma: a Case Report, Review of the Literature and Proposal for Two Clinicopathological Types

P.A. Pahapill, D.A. Ramsay and R.F. Del Maestro (London, Ontario)

A case of pleomorphic xanthoastrocytoma (PXA), a low grade glioma of childhood with a reported favourable prognosis, is presented. A literature review of 79 PXA cases is described and confirms a favourable prognosis in at least $80 \%$ of cases. The sex ratio in the reported cases is almost equal and the median age at the time fo diagnosis was 15 years. $79 \%$ of the patients presented with seizures. Nine of the 15 deaths from PXA are associated with histological evidence of necrosis at presentation or in a recurrent tumour. Two types of PXA can be recognized on the basis of the absence or presence of necrosis: "type I", without necrosis, has a good prognosis ant "type II", with necrosis, has a poor prognosis. "Type I" PXA has the potential to progress to "type II" PXA. Optimal treatment for "type I" PXA includes primary surgical resection with re-operation for recurrent tumour. The role of surgery in "type II" PXA or the role of radiotherapy in "type I" or "type II" PXA remains unclear.

\section{0-6}

\section{Medulloblastoma in Children}

M. Vassilyadi, J.P. Farmer, J.L. Montes, S. Choi, J.E. Blundell and K. MEAGHER-VILlemure (Montreal, Quebec)

A retrospective analysis of medulloblastoma in 103 children treated at the Montreal Neurological and Children's Hospitals from 1950 - 1992 was performed, and the results compared with the recent literature. There were 20 children $<$ three years of age, and 83 above.

The ratio of males to females was 1.6:1, however, there was no gender difference in survival probability. There was no age difference in survival after adjustments were made for the children that died within the first three weeks of admission. Overall one-year survival was $77 \%$, and five-year survival was $53 \%$. Before 1977 , five-year survival was $47 \%$, after it was $62 \%$; this was significant only for the older children where the survival probability increased from $41 \%$ to $70 \%$.

Tumour site nor Chang's T stage correlated with survival; $M$ stage was a strong predictor of mortality. Desmoplastic histology showed a better, but not statistically significant, survival compared with classic histology. Shunting had no influence on survival. Radical subtotal resection was no different than complete resection. Chemotherapy showed no survival benefit. Standard dose radiation therapy demonstrated a greater, but not statistically significant, survival probability than low dose radiation therapy.

\section{0-7}

\section{Late Recurrence of Cerebellar Astrocytoma: An Update}

\section{A.L. AmaCher (Danville, Pennsylvania)}

Twenty-seven consecutive patients with juvenile cerebellar astrocytoma eligible for 10-year follow-up (10 - 45 years) following resection provided this data: overall survival $-96.3 \%$; recurrence rate at 10 years - $11.5 \%$; recurrence rate now $34.5 \%$; all three patients followed for $30+$ years have suffered recurrences. Longest intervals to recurrence are 20,30 and 37 years. There have been no multiple recurrences. Our experience indicates that the recurrence risk for juvenile cerebellar astrocytoma: increases with time; is not prevented by perceived gross total removal; is not diminished by radiotherapy. Four of 18 cystic lesions and five of nine microcystic tumours have recurred. Selected cases of recurrence will be discussed. The pertinent literature is reviewed. 
$0-8$

\section{Evidence for a High Free Radical State in Low Grade Astrocytoma}

D.F. Louw, G.R. Sutherland, R. Bose and A.A.F. Sima (Calgary, Alberta; Ottawa, Ontario; Ann Arbor, Michigan)

It is posited that reactive oxygen species may play an inductive role in neuro-oncogenesis. However, data pertaining to the redox state of astrocytomas are quite limited, prompting us to undertake this study. Intraoperative, snap-frozen samples were obtained from the surface and core of seven benign and 11 malignant astrocytomas. Small portions of each specimen were fixed in $10 \%$ neutral formalin or cacodylate-buffered glutaraldehyde. Standard techniques were employed to measure lipid peroxidation by estimating thiobarbituric acid-reactive substances (TBARS), and glutathione (TGSH) assays were determined. Light microscopy was performed to define the relevant histopathology, and electron microscopy was applied to quantitate peroxisomal content. TBARS values for benign astrocytomas were significantly elevated in comparison to malignant lesions, as was the case for TGSH. This discrepancy was especially marked at the tumour surface. Peroxisomes predominated in the benign category. We infer malignant dedifferentiation as a possible consequence of this reactive metabolite burden, as well as speculate on the role of seizures and astrocytoma glutamate receptors in the initiation of free radical cascades. The therapeutic and teleologic implications are not inconsiderable.

\section{0-9}

Effects of ${ }^{125}$ I Brachytherapy on the Cell Cycle and Histopathology of Recurrent, Malignant Astrocytomas

\section{S.N. Siddigi, J. Provias, N. Laperriere and M. Bernstein} (Toronto, Ontario)

${ }^{125}$ I Brachytherapy has been used to provide high radiation doses to localised regions of recurrent malignant astrocytomas in an attempt to improve the length of patient survival. In this study, we examined the histological effects of ${ }^{125} \mathrm{I}$ Brachytherapy on recurrent malignant astrocytomas and the effect on the cell cycle of tumour cells using proliferating cell nuclear antigen (PCNA) immunostaining. All patients in this study had been initially treated within a randomised, controlled study with or without ${ }^{125}$ I Brachytherapy following initial surgical resection and external beam radiation $(50$ Gy in 25 fractions). Specimens from 24 patients later reoperated for "recurrence" were used in the present analysis. ${ }^{125}$ I Brachytherapy reduced certain histological features prognostic for tumour progression: cellularity, pleomorphism, vessel hyperplasia and degree of mitosis ( $p<0.05$ ). The degree of "mitosis" (marker for the "mitotic, 'M",, phase) and "PCNA index" (marker for the late $\mathrm{Gl}$ and $\mathrm{S}$ phase) provide complementary data on the cell kinetics of the tumour. PCNA immunostaining was lower in the 125I Brachytherapygroup $(34.6 \pm 8.2 \%$, mean \pm SER $)$ compared to the control, non-implant group $(68.2 \pm 3.5 \%)$. However, ${ }^{125} \mathrm{I}$ Brachytherapy produced a more dramatic reduction in mitotic figures (mean histological score $=0.0 \pm 0.0$ ). These findings indicate that treatment with ${ }^{125}$ I Brachytherapy significantly reduces the proliferative capacity of a recurrent malignant astrocytoma as measured by a reduction in mitosis, PCNA labelling of nuclei, cellularity, pleomorphism, and vessel hyperplasia.
Moreover, the $\mathrm{M}$ phase of the tumour cell cycle appears most sensitive to ${ }^{125}$ I Brachytherapy.

\section{0-10}

\section{Cerebrovascular Effects of Stereotactic Radiosurgery in the Treatment of Brain Tumours: a CT Study}

\section{T.-Y. Lee, R.F. Del Maestro, J. Bennett and R. Kozak (London, Ontario)}

Vascular damage is an important limiting factor in the treatment of primary and metastatic brain tumour with stereotactic radiosurgery. It is believed that brain capillaries are damaged by ionizing radiation leading to ischemia, edema, and finally tissue (tumour) necrosis. We have developed a dynamic CT scanning method to assess the degree of damage by the simultaneous invivo measurement of cerebral blood flow (CBF), blood volume (CBV) and blood-brain barrier permeability (K).

The method was based on the compartmental and stochastic analysis of the cerebral contrast enhancement curves obtained by dynamic CT scanning following intravenous injection of contrast agent. As described before, ${ }^{1,2}$ the compartmental analysis yielded CBV and $\mathrm{K}$, while the stochastic analysis yielded the mean transit time $\left(t_{m}\right)$ of the brain. CBF was then calculated according to the Central Volume Principle as the quotient of CBV and $t_{m}$

Six patients were studied before and at 2, 6, 12, and 26 weeks after stereotactic surgery. Our results showed that: (1) tumour necrosis had decreasing CBF and CBV; (2) tumour recurrence had increasing $K$ and $C B F$; and , (3) normal tissue necrosis had increasing $\mathrm{K}$ and decreasing $\mathrm{CBF}$.

'I Yeung \& T.-Y. Lee et al. J NeuroOncol 14, 177-187; ${ }^{2}$ I Yeung \& T.-Y. Lee et al. J NeuroOncol 18, 53-60.

\section{0-11}

\section{Basement Membrane Macromolecules Modulate the Degradation of Type IV Collagen by C6 Astrocytoma}

\section{J. Keshmirian, Warren McDonald and Rolando F. Del} MAESTRO (London, Ontario)

Background: A crucial step in tumour-associated angiogenesis is the degradation of collagen type IV (Col IV) residing in the vascular basement membranes (BM).

C6 astrocytoma cells in monolayer culture and in vivo have been shown to release a number of enzymatic activities capable of degrading Col IV. In this study we addressed the following question: What is the influence of reconstituted BMs (RBM) as well as individual BM components on the ability of $\mathrm{C} 6$ cells to degrade Col IV? Methods: C6 cells were grown in culture wells coated with laminin (Lam), Fibronectin (Fn), Col IV and reconstituted BM (RBM), matrigel. Conditioned medium was then removed at different time intervals and assayed for collagenase IV activity. Results: The ability of C6 cells to degrade Col IV was suppressed by all the individual components of BMs. Compared to controls, Lam, Fn and Col IV reduced the proteolytic activities by up to $50-75 \%$. However, RBM which is a 3 dimensional meshwork of molecules closely resembling in-vivo BMs did not influence or increase the ability of C6 cells to degrade Col IV and other proteins. Conclusions: The ability of C6 astrocytoma cells in monolayer culture to degrade Col IV is down-regulated in the presence fo Lam, Fn and Col IV. Growth 
factors sequestered in the RBM when released may have upregulated collagenase IV activity. The ability of individual BM components to suppress the collagenase IV activity of C6 astrocytoma cells may help explain the failure of astrocytomas to metastasize. The therapeutic use of individual basement membrane macromolecules as an anti-angiogenic strategy is presently being explored.

\section{POSTER PRESENTATIONS}

\section{SESSION I}

(Thursday, June 22, 07:00-17:00,

Authors Standing By 13:00 - 14:00)

\section{PEDIATRIC NEUROLOGY $(P-01$ to $P-13)$}

\section{P-1}

Assessment of the Appropriateness of Visitation Sessions in the Meningomyelocele Clinic By Way of a Patient Focused Program Evaluation

G.K. Chambers, D. Douglas Cochrane, William Arnold and BEVERLY IRWIN (Vancouver, British Columbia)

The Meningomyelocele Clinic/program at B.C.'s Children's Hospital has been serving the needs of spina bifida children since 1969; in 1994 seeing over 330 patient/families. The clinic provides the patient with urgent and routine clinical assessments at regular intervals throughout childhood and adolescence in a multidisciplinary environment. The frequency of visits and the value of the clinic to the patient/families had never been assessed. The purpose of the study was to assess the appropriateness of visitation sessions in the clinic. Routine visit frequency, patient satisfaction with clinic health services, patient assessment of the specific value of visitation sessions and health professionals satisfaction issues were evaluated. 65 families were interviewed and asked to fill out a questionnaire developed locally by the clinic professionals. Clinic services met patient/ families needs, the medical staff and nursing component were seen as valuable resources for the patient/families and most patient/families felt well informed about spina bifida. Families did not see the role of the paramedical staff as being as useful as the medical staff. The study demonstrated the main value of the clinic being the educational component. The program is seen to be operating at optimal frequency and giving health care value to the patient/families attending the clinic.

\section{P-2}

\section{Thrombotic Thrombocytopenic Purpura: a Treatable Cause of Childhood Encephalopathy}

D.W.M. Webi, B. Lawlor, L. Wadsworth and A. Hill (Vancouver, British Columbia)

Thrombotic thrombocytopenic purpura (TTP) is a rare disorder in childhood which, if untreated is associated with a high mortality. Recent studies of adults with TTP have revealed a substantial benefit from plasma exchange. However, experience with this treatment in childhood is limited.

A previously healthy six-year-old boy was referred with progressive lower limb cellulitis following minor trauma. Within hours of admission, he required ventilatory support because of recurrent generalized seizures and a decreasing level of consciousness. He continued to have refractory generalized seizures. The EEG was consistent with a diffuse encephalopathy. Cranial CT scan and CSF analysis were normal. Thrombocytopenia was noted six hours after admission and by 48 hours he had evidence of a microangiopathic hemolytic anaemia. Coagulation studies were normal. He developed mild renal impairment and a urinary sediment. Plasma exchange was initiated on day three. After the second exchange, he responded to verbal commands and seizures ceased. Neurological status improved and anticonvulsants were weaned. Three weeks following admission, he was discharged with a normal neurological examination.

Thrombotic thrombocytopenci purpura is a potentially treatable cause of life threatening childhood encephalopathy.

\section{P-3}

\section{Basal Ganglia Infarction Associated with HHV-6 Infection}

D.W.M. WebB, E. Thomas and B. Bjornson (Vancouver, British Columbia)

Primary infection with HHV-6 infection occurs predominantly in early childhood and is associated with exanthem subitum (roseola). It is associated also with febrile seizures and may cause encephalitis.

A previously healthy Chinese boy was admitted with a fever and history of increasing drowsiness. He was localising to pain and had eye opening but no verbal response. He had meningism and generalized hypertonia. Brain stem relfexes were intact. He subsequently developed clinical and biochemical evidence of an anicteric hepatitis. His CSF showed a pleocytosis with normal glucose and a modestly raised protein. Cultures were negative for bacterial and viral pathogens but there was serological evidence of acute HHV-6 infection. Serial CT scans revealed focal infarction of both basal ganglia. His neurological examination has remained abnormal with evidence of a spastic quadriparesis and mild dysarthria three months after discharge.

HHV-6 should be sought in children of all ages who present with unexplained meningoencephalitis. This infection may be associated with severe neurological sequelae.

\section{P-4}

\section{Infant Migraine Response to Pizotyline}

\section{S.D. LEuIN (London, Ontario)}

Two cases of periodic syndrome, thought to be migraine, presenting in the first few months of life and with a dramatic response to pizotyline, are presented.

CASE I A five-year-old girl presented at two weeks of age with apparent colic and severe vomiting. Between 3 - 5 months she required daily gravol. As she grew older, the bouts of recurrent vomiting reduced in frequency to three per week, usually 
after car rides. The maternal grandmother, grandfather and aunt had classical migraines. Neurological examination and CT scan of the head were normal. She was treated with pizotyline increasing to a maximum dose of $1 \mathrm{mg}$ and the episodes stopped.

CASE 2 A two-and-a-half-year-old girl presented at four months with recurrent episodes of vomiting lasting 30 minutes associated with lethargy for several hours afterwards. These were associated with foul-smelling diarrhea. There was one severe episode per month and one mild episode per week. Mother had classical migraine. General and neurological examinations were entirely normal. Investigations included CBC, urinalysis, electrolytes, ammonia, plasma and urine amino acids, urine organic acids, upper GI series and small bowel barium follow through study all of which were normal. The episodes stopped completely on pizotyline $0.125 \mathrm{mg}$ qhs.

These antecdotal observations suggest pizotyline is useful in the treatment of infant migraine.

\section{P-5}

\section{Clinical Presentation of Syringomyelia in Childhood: Report} of Six Cases

\section{V.V. Vedanarayanan, Andrew Parent and Owen B. Evans} (Jackson, Mississippi)

Symptomatic syringomyelia is rare in childhood and usually presents in adult life. These are often associated with Arnold Chiari malformations. We are reporting six consecutive cases of syringes which were not associated with Arnold Chairi malformations and presented with variable clinical features.

Two children presented at ages 20 months and seven years had terminal syringomyelia and tethered spinal cord. The 20month-old presented with unexplained toe walking and was delayed with onset of walking. The seven-year-old child presented with dysesthesia in right foot and leg with refusal to walk which started subacutely. Symptoms resolved rapidly with dethereing of the cord and shunting of the syrinx.

Two children presented with syrinx in the lower cervical and upper thoracic cord. A 13-year-old girl presented with intermittent numbness and parasthesias in the left arm and chest, and she had mild levoscoliosis of the spine in the thoracic region. A 12-year-old boy presented with levoscoliosis and no other symptoms.

Two girls aged five and six presented with secondary enuresis and no objective neurological findings. They both had small syringes in the lower cervical cord. We conclude that the clinical presentation of syringomyelia in childhood can be subtle and variable. Evaluation of the spinal cord would be indicated for subjective symptoms and gait abnormalities in the absence of an obvious cause.

\section{P-6}

Cerebral Infarction in Childhood: Is the Left Hemisphere More Susceptible to Ischemic Injury?

N. Vingerhoets-Clerc, B. Bjornson and K. Poskitt (Vancouver, British Columbia)

Reasons for the lateral distribution of cerebral infarcts have not been well elucidated, but could include gender effects; there- fore, we sought to categorize cases identified at a tertiary referral centre.

We did a retrospective study of pediatric cases retrieved from the health record and radiology databases of British Columbia Children's Hospital in Vancouver for the period 1984 - 94. CT and MRI films were independently reviewed and classified by a neuroradiologist. Cases involving cerebral trauma, surgery, infection, tumour malformation, AVM, aneurysm or multiple vasculitic lesions were excluded.

230 cases of radiologically confirmed ischemic infarction were retrieved. The anatomic distribution of lesions was: cerebral hemispheres 223 , brainstem four and cerebellum three. Cerebral infarcts included 144 unilateral cases and 79 bilateral cases. The distribution of confirmed cerebral infarcts by gender was 142 male $(62 \%)$ and 81 female $(38 \%)$. Unilateral cerebral infarcts were more often located in the left hemisphere $(58 \%)$ than the right (42\%). Among females, only 18 unilateral infarcts $(35 \%)$ were located in the right hemisphere. The left hemisphere may be more susceptible to injury, or may produce more obvious disability, leading to diagnostic bias in its favour.

\section{P-7}

\section{Acute Hemorrhagic Leukoencephalitis in Siblings in Infancy}

P. Diadori, H.Z. Darwish, C.B. Van Orman, A. Pinto, C.L. Trevenen and D. Mueller (Calgary, Alberta)

Objective: Report of a pair of siblings who died of acute hemorrhagic leukoencephalitis (AHL) in infancy. Affected areas on cranial CT scan is different from adults. Background: Adult AHL has prominent involvement of hemispheric white matter on CT and pathology. No familial or sibling case has ever been reported. Design/Method: A review of AHL in the English literature and comparison to our cases. Result: Two male siblings presented at 5 (case 1) and 8 (case 2) months of age with an upper respiratory tract infection followed weeks later by an explosive encephalopathy characterized by recurrent seizures and coma. Systemic shock was absent. Cranial CT scans were impressive for decreased attenuation of thalami. At autopsy influenza A (case 1) and RSV (case 2) was isolated from the respiratory tract but not the central nervous system (CNS). Thalamic hemorrhage and fibrinoid necrosis of small vessel walls with neutrophil transmigration, perivascular hemorrhage and demyelination was found. Conclusion: This is the first report of AHL in infant siblings. Involvement of thalami has been reported in only one other child. Cranial CT findings within appropriate clinical context indicates childhood AHL. We speculate that a recessively inherited disorder in these infants predisposed them to an abnormal augmented autoimmune response in the CNS to a systemic viral agent.

\section{P-8}

Spondylometaphyseal Dysplasia With Progressive Spastic Diplegia

\section{J. MAH and D.B. SINCLAIR (Edmonton, Alberta)}

Spondylometaphyseal Dysplasia (SMD) is a heterogenous group of diseases in which the vertebrae and the metaphyses of the tubular bones are affected. Several different forms of this 
condition are now described. We report a four-year-old girl with Kozlowski type of SMD who presented with progressive spastic diplegia. The clinical and radiological features are described. This is the second reported case in the literature of a child with SMD and progressive spastic diplegia. Spondylometaphyseal Dysplasia with progressive spastic diplegia represents a new variant of SMD. The occurrence of these two rare conditions together in this group of patients suggests a genetic linkage which may be valuable in identifying the genetic locus and understanding the pathophysiology of this disease.

\section{P-9}

\section{MRI Findings in Ataxia-Telangiectasia}

\section{P. LIDMAN and D.B. SinClaIR (Edmonton, Alberta)}

Ataxia-Telangiectasia is an autosomal recessive disorder of childhood characterized by oculo-cutaneous telangiectasia, progressive cerebellar ataxia, immune deficiency and recurrent bronchopulmonary infection. We report two children with Ataxia-Telangiectasia and discribe the Magnetic Resonance Imaging (MRI) findings. Both patients were at the same stage of their disease and had diffuse cerebellar atrophy on MRI. One patient, in addition, had diffuse white matter changes on a T2 weighted MRI Scan suggestive of a leukodystrophy. An extensive workup for leukodystrophy in that patient was negative. This is the second reported case in the literature of white matter changes on MRI mimicking a leukodystrophy. There is a spectrum of radiological findings in Ataxia-Telangiectasia. The MRI is characterized by diffuse cerebellar atrophy in this condition but may include diffuse white matter changes on T2 weighted MRI Scans. Ataxia-Telangiectasia should be considered in the differential diagnosis of patients with diffuse white matter changes on MRI

\section{P-10}

\section{Facial Nerve Paralysis of Dental Origin in Children}

\section{Gerald Friedman (Toronto, Ontario)}

Facial Nerve Paralysis (FNP) is a relatively common condition with many causes. Although most often idiopathic, secondary causes of FNP such as trauma, infections (Lyme Disease, Herpes Zoster), diabetes, pregnancy, and neoplasms must be excluded. Dental causes of FNP are extremely rare but known to occur following superior and inferior dental blocks, as well as oral infections.

Dental causes of FNP have not been previously reported in children. Two cases of FNP of dental origin in children are described. The underlying mechanism of action and pathogenesis are discussed

\section{P-11}

\section{Brain Tumours in Children}

Daniel L. Keene, E. Hau and E. Ventuyra (Ottawa, Ontario)

A retrospective chart review from 1976 to 1993 of patients diagnosed as having a brain neoplasm at the Children's Hospital of Eastern Ontario was carried out.

190 tumours occurred during the period (approximately 10 per year). $52 \%$ were supratentorial and $48 \%$ infratentorial. Of the supratentorial tumours, $50 \%$ were in the hemisphere; $8 \%$ were optic gliomas; midline tumours were $42 \%$. Of the infratentorial tumours, $23 \%$ were brainstem; $77 \%$ were cerebellar.

Hemispheric tumours presented with a mean age of 9.5 years as either seizures or focal long track signs. The histological tumour type consisted mainly of astrocytomas (30\%), ganglioma (30\%), high grade malignancy $(20 \%)$.

Optic gliomas had a mean age of presentation of 8.5 years. Visual impairment was the main symptom.

Midline tumours presented at a mean age of 10.3 years with insidious presentations. $34 \%$ of the midline tumours were in the pineal region; $41 \%$ in the suprasellar region of which craniopharyngioma was the most common (59\%) followed by astrocytoma $(29 \%)$. Pituitary adenomas, thalamic and intraventricular masses making up the remainder.

Brain stem tumours made up $11 \%$ of the total with the mean presenting age of 7.3 years. Presenting symptoms were cranial neuropathies and long track signs.

Cerebellar tumours made up $37 \%$ of the total tumours. The mean presenting age was 7.5 years. Headache, vomiting and signs of ICP were found in the majority of cases but not all. Histological diagnosis consisted of PNET (38\%), astrocytomas $(46 \%)$, and ependymoma (9\%).

\section{P-12}

\section{Ganciclovir Therapy in Symptomatic Congenital CMV} Infection: Initial Experience

B.L. Banwell, D.L. MacGregor, L. Ford-Jones and S. BlASER (Toronto, Ontario)

Symptomatic congenital cytomegalovirus (CMV) infection causes central nervous system (CNS) morbidity in $90 \%$ of affected children. Ganciclovir has been shown to improve outcome in infected immunocompromised patients. We report the neurologic and radiologic outcome in four symptomatic congenitally infected patients treated within 24 days of age with a six week course of Ganciclovir (10 - $12 \mathrm{mg} / \mathrm{kg} /$ day) and compare their outcome to historical controls.

Hepatosplenomegaly, petechiae and thrombocytopenia were noted in all four children at birth, none had microcephaly or chorioretinitis. Neuroimaging was consistent with congenital CMV (periventricular lucencies, intracranial calcification, or pachygyria) in all four cases.

Patient one (three years, eight months) has progressive microcephaly seizures, and significant developmental and motor delay. Patient two (two years, two months) is normocephalic, developing normally and has a normal audiologic exam. Patient three (four years, four months) has progressive microcephaly, developmental delay, right hemiparesis, and profound sensorineural deafness. Patient four (12 months) has shown early motor and language delay.

The CNS sequelae in our patients is consistent with the literature. Our preliminary experience suggests that CNS prognosis is not improved by Ganciclovir therapy. 
Acute Viral Encephalitis Registry: The First Year

Melinda Davie, D.L. MacGregor and L. Ford-Jones (Toronto, Ontario)

Acute viral encephalitis (encephalopathy plus two or more of fever, seizure, focal neurologic findings, EEG abnormality, neuroradiologic abnormality, CSF pleocytosis) in children is usually a diagnosis of exclusion because only a limited number of viruses can be routinely identified with current diagnostic methods. To improve our ability to reach a diagnosis and collect epidemiologic data on neurologic outcome a prospective registry was established. Clinical date, sera and CSF were analyzed with sera and CSF banked for future serology and polymerase chain reaction (PCR).

The 22 subjects were patients admitted (four months - 17 years) with no known previous neurologic disease who met the above criteria. Paired sera was done for adenovirus, arboviruses, mycoplama, herpes and enterovirus. PCR was also done for herpes and enterovirus. All patients underwent neurolradiologic imaging and EEG.

Four children (18\%) had specific diagnoses; powassan (1), herpes simplex (1), mycoplasma (2). One child died. Morbidity included significant motor deficit (1), hearing loss (1), behaviour problems (2) and recurrent seizures (2).

The registry may improve identification of the etiology and longterm neurological outcome of acute viral encephalitis.

\section{NEUROSURGERY - TRAUMA $(P-14$ to $P$-2 $I)$}

\section{P-14}

Delayed Ischemic Neurological Deficit From Closed Head Injury

M. Vassilyadi, J.L. Caron, P.M. Richardson and R.M. Ford (Montreal, Quebec)

Delayed ischemic neurological deficit from closed head injury is uncommon. When it occurs, the etiology is posttraumatic cerebral artery narrowing, usually associated with cisternal, subarachnoid, or intracerebral blood.

A 21 -year-old girl was involved in a motor vehicle accident sustaining multiple facial and basal skull fractures, worse on the right side. Brain CT revealed subarachnoid hemorrhage, mostly in the perimesencephalic cisterns, and cerebral edema without any midline shift. Intracranial pressure was never greater than $15 \mathrm{mmHg}$.

She improved to the point of obeying commands until the ninth day when she deteriorated to the point of decerebrate posturing. She sustained a mild hypotensive episode following reintubation. Brain CT revealed bilateral hemispheric ischemia, more marked on the right side, and totoal resolution of the subarachnoid hemorrhage. Doppler studies showed increased velocities in both internal carotid arteries. Angiography showed narrowing of both supraclinoid internal carotid arteries, as well as the $\mathrm{Al}$ and $\mathrm{Ml}$ branches, with no extracranial arterial injury. With Nimodipine and sustained hypertension, she gradually improved. Vulnerability to ischemia provides one explanation for the poor prognosis associated with hypotension after head injury.

\section{The Role of Voltage Gated Potassium Channels in Axonal Dysfunction After Spinal Cord Injury}

\section{Michael G. Fehlings and RAAd NASHMI (Toronto, Ontario)}

Abnormal activiation of $\mathrm{K}^{+}$channels is thought to play a key role in posttraumatic axonal dysfunction after spinal cord injury. In this study, we have examined which $\mathrm{K}^{+}$channel subtypes underly this process.

A $2 \mathrm{~cm}$ length of dorsal column (DC) was isolated from the spinal cord of adult rats $(n=51)$, pinned in vitro and perfused with oxygenated Ringer. Field potentials were recorded from glass microelectrodes (150 mM KCL; 5-10 M $\Omega$ ). A clip injury ( $2 \mathrm{~g}$ closing force; $15 \mathrm{sec}$ ) was applied to the DC segment. The presence of "fast" $K^{+}$channels ( $f_{1}$ and $f_{2}$ subtypes) was investigated with 4-aminopyridine (4-AP) and $\alpha$-dendrotoxin ( $\alpha$-DTx, $500 \mathrm{nM}$ ). Dendrotoxin-I (DTx-I, $100 \mathrm{nM}$ ) was used to probe for the $f_{1}$ subtype of the "fast" $K^{+}$channel. Tetraethylammonium (TEA) and $\mathrm{CsCl}$ were used to examine for delayed rectifier ("slow") and inward rectifier (IR) channels.

Infusion of a-DTx and 4-AP resulted in a reduction in amplitude of the compound action potential of injured axons to $69.8 \pm$ $4.2 \%(\mathrm{p}<0.00025)$ and a prolongation of the absolute refractory period by $60.7 \pm 13.2 \%$ ( $p<0.0005)$. However, no differences were observed in the conduction properties of injured and noninjured axons with DTx-I, TEA or CsCl.

In conclusion, our results suggest that the $f_{2}$ subtype of the "fast" $\mathrm{K}^{+}$channel contributes to posttraumatic axonal dysfunction. In contrast, the $f_{1}$ subtype "fast" $K^{+}$channel, the TEA-sensitive "slow" $\mathrm{K}^{+}$channel and the $\mathrm{CsCl}$-sensitive IR channel do not mediate this phenomenon.

\section{P-16}

Role of Sodium in the Pathophysiology of Secondary Spinal Cord Injury

Michael G. Fehlings and Sandeep Agrawal (Toronto, Ontario)

Study Design: Experimental study using an in vitro model of compressive injury to isolated adult rat dorsal column axons.

Objectives: To examine the role of extracellular $\mathrm{Na}^{+}\left(\mathrm{Na}_{\mathrm{c}}{ }_{\mathrm{c}}\right)$ in mediating secondary injury to spinal cord axons after compressive trauma. The mechanisms of intracellular sodium entry were examined using ion substitution techniques and pharmacological blockers.

Background: There is evidence that intracellular $\mathrm{Na}^{+}$entry potentiates hypoxic/ischemic cell death by causing cytotoxic cell swelling, intracellular acidosis and gating of $\mathrm{Ca}^{++}$entry through reverse activation of the $\mathrm{Na}^{+}-\mathrm{Ca}^{++}$exchanger (NCE). In the present study, we have examined the role of $\mathrm{Na}^{+}$in the pathophysiology of spinal cord injury (SCI).

Methods: Dorsal column segments isolated from the thoracic cord of adult rats $(n=40)$ were pinned in a recording chamber and superfused with oxygenated Ringer. Field potentials (CAP) were recorded from glass microelectrodes $(150 \mathrm{mM}$ $\mathrm{KC}$; ; 5-10 M $\Omega$ ). Injury was accomplished in vitro by compression with a clip (closing force $2 \mathrm{~g}$ ) for $15 \mathrm{sec}$. The effect of zero $\mathrm{Na}^{+}$(substitution with $\mathrm{NMDG}^{+}$, the $\mathrm{Na}^{+}-\mathrm{H}^{+}$exchange blocker amiloride, the $\mathrm{Na}^{+}$channel blocker procaine and the NCE 
blocker benzamil on CAP recovery after compressive injury were assessed.

Results: Pretreatment with zero $\mathrm{Na}^{+}$, amiloride and procaine conferred significant neuroprotection $(p<0.05)$. In contrast, the NCE blocker benzamil was ineffective in attenuating secondary injury.

Conclusions: Reduction of extracellular $\mathrm{Na}^{+}$, inhibition of the $\mathrm{Na}^{+}-\mathrm{H}^{+}$exchanger or blockade of voltage gated $\mathrm{Na}^{+}$channels is neuroprotective after SCI. The mechanism of $\mathrm{Na}^{+}$-associated cytotocity does not involve reverse gating of the NCE.

\section{P-17}

\section{Arteriovenous Lactate Difference in Severe Human Traumatic Brain Injury}

\section{J.I.M. Brown, S.J. KonaSIEwicz, A. Baker and R.J. Moulton (Toronto, Ontario)}

Introduction: Ischaemia is an important potential mechanism of secondary damage after traumatic brain injury (TBI). Ischaemic brain metabolises glucose by glycolysis, producing lactic acid which may cause further brain injury. Net cerebral lactate production can be observed as an arterial-jugular venous lactate difference $\left(A V D L=\right.$ lactate $_{A}-$ lactate $\left._{\mathrm{V}}\right)$ after TBI. We assess the usefulness of this simple test as an index of cerebral ischaemia and as an acute prognostic indicator by correlation analysis of AVDL and measures of cerebral ischaemia, clinical outcomes and quantitative electrophysiologic parameters.

Methods: From July 1987 to January 1995, intensive monitoring of 106 patients with sever TBI (GCS $\leq 8$ after resuscitation) included twice daily AVDL, $A V D O_{2}, \mathrm{CBF}\left(\mathrm{N}_{2} \mathrm{O}\right.$ diffusion method) and CSF glutamate levels (35 patients), continuous EEG's and hourly somatosensory evoked potentials (SSEP's) for an average of five days. Clinical outcomes were Glasgow Coma Score on day 14 (GCS14) and Glasgow Outcome Score (GOS) after three months. Quantitative measures of EEGs were total spectral power and percent slow wave activity and for SSEPs, summed peak to peak amplitudes. A statistical software package was used for correlation analysis.

Results: AVDL was not correlated with other indicators of ischaemia $\left(\mathrm{AVDO}_{2}\right) \mathrm{CMRO}_{2}, \mathrm{CBF}, \mathrm{CSF}$ glutamate), electrophysilogic indices or changes in these indices or with clinical outcomes. Furthermore, no correlations were observed in separate analyses of different outcome subgroups or of early $(<48$ hrs) and late ( $>48 \mathrm{hrs}$ ) epochs post injury.

Conclusions: Although anaerobic cerebral metabolism of glucose can be detected as net lactate production (negative AVDL) in the acute period after TBI, it is not a useful predictor of clinical or electrophysiologic outcomes and is not correlated with other indicators of ishaemia.

\section{P-18}

Craniocerebral Gunshot Wounds: a South Saskatchewan Experience

Michael Peter Bruce Kilburn, R. Griebel and G. Goplen (Saskatoon, Saskatchewan)

Craniocerebral gunshot wounds are a major cause of morbidity and mortality in North America each year. These injuries occur as a result of suicide attempts or from assault. In a retro- spective study of 36 patients seen at the Plains Health Care Centre between 1980-1992 we undertook an effort to examine the epidemiological distribution, outcomes and prognostic indicators in craniocerebral gunshot wounds. We recognized five outcome categories: complete functional recovery; moderate disability with independent activities of daily living (ADLs); severe disability with dependence on others for ADLs; persistent vegetative state; and death. We examined how these outcomes could be determined retrospectively based on presenting Glasgow Coma Scale (GCS), radiological findings, pupillary response, site of injury, and age. We then examined whether craniotomy in selected patients contributed to improved or worsened outcome. From our data we concluded no patient with bilaterally fixed dilated pupils, GCS $<11$, a wound through the geographic centre of the brain, intraventricular or subarachnoid bleeding or a marked midline shift survived their injury. Factors which predicted a favorable outcome included: wounds from assault as opposed to suicidal injuries, nonfrontal/nonparietal wounds, absense of mass lesions on CT scanning, GCS > 11 and wounds not through the geographic centre of the brain.

\section{P-19}

\section{Assessing Traumatic Injury to Axons in an In-vitro Cell Culture System: Preliminary Results}

\section{FAYAZ and C.H. TATOR (Toronto, Ontario)}

To characterize and understand the early events in acute injury to the spinal cord, our laboratory has developed an invitro model of axonal injury. This approach has given us the ability to examine and assess individual cellular injury mechanisms, especially as they relate to axonal injury initiation and progression.

Superior cervical ganglia from newborn rats are cazymatically dissociated and plated on a polyomithin-laminin matrix. The matrix is scratched prior to plating, yielding 20 parallel tracks, $250 \mu \mathrm{m}$ wide. These principal neurons are plated in the center of the dish using specially designed compartmented chambers, with only neuritic extensions towards the periphery. The tracks guide the neurites to grow linearly, and some show fasciculation, yielding a thick network of neurites on each track. Axonal injury is made using a custom designed electrical motor with a magnetically driven, vertically moving shaft. The force of injury can be varied according to the shaft velocity. This device has produced consistent transection of the neurites at desired locations from the cell somata.

Following complete transection injury to these neurites, the proximal injury site initially displayed some thickening, progessing to retrograde degeneration or dieback within the first 30 minutes, and continuing for up to 2 hours. The degree of degeneration ranged from 85 to $150 \mu \mathrm{m}$. In all transected tracks, growth cones were noted emerging from the proximal end at 2 hours following injury, with good definition of further growth observed by 6 hours. By this time, evidence of anterograde neurite degeneration was fairly marked, consisting of some initial thickening with progression thereafter to a pattern of fragmentation. By 18 hours, all injured tracks showed at least partial neuritic traversing of the injury site, and by 30-36 hours there was complete crossing of the injury site. Light and scanning electron microscopy showed that the regenerating neuritic arbour was morphologically distinct from the parent fibers in both calibre and number. 
The readily observed degenerative and regenerative changes in post-traumatic neurites in vitro makes this model of acute axonal injury ideal for examining calcium homeostasis with fluorescent ratio imaging or confocal imaging, and for assessing the nature and extent of the neuritic cytoskeletal disaggregation following injury. Studies are in progress to assess the impact of various chemical derangements on the regenerative ability of these neurites following traumatic injury.

\section{P-20}

Conservative Treatment of Spontaneous Spinal Epidural Hematoma: a Case Report and Review of the Literature

\section{P.A. Pahapill and S.P. Lownie (London, Ontario)}

Spontaneous spinal epidural hematoma is a rare cause of spinal cord compression that left untreated produces a permanent neurological defict. The standard form of therapy is prompt surgical evacuation of the hematoma, which usually results in good neurological recovery. We present the case of a spontaneous spinal epidural hematoma producing neurological deficits that resolved without surgical intervention. Only 6 such cases have been reported previously. In most of these cases significant and sustained neurological recovery had occurred within 8 hours of the impairment of walking. This may be a reason to consider conservative treatment. The potential role of non-surgical treatment for spontaneous spinal epidural hematomas is discussed.

\section{P-21}

A New Superior Technique for Removal of Herniated Lumbar Disc: Endoscope and Nucleotome Combination

\section{David A. Ditsworth (Los Angeles, California)}

After performing several hundred percutaneous discectomies since 1987 using various techniques and equipment, this author has developed a new and superior technique for combining the standard Nucleotome with an innovative, highly effective working channel endoscope.

Since November '93, thirty-two patients have had percutaneous lumbar discectomies with local anesthesia performed with a newly developed endoscope, consisting of a $2.8 \mathrm{~mm}$ working channel combined with a 6,000 pixel fiber-optic scope, which is passed through a $4.2 \mathrm{~mm}$ diameter outer cannula. A series of instruments, including forceps, a suction cannula, an obturator and a $2.5 \mathrm{~mm}$ Nucleotome are introduced through the working channel, providing not only internal decompression, but also removal of extruded or separated fragments located outside the disc space. The combination internal and external disc access provides an excellent comprehensive new approach.

All 32 patients had sciatica as the principal complaint, with duration of symptoms from several months to many years. All patients had positive MRI or CT scans, failed conservative care and had clinical findings of a lumbar disc rupture (positive straight leg raising, motor, sensory, or reflex abnormalities).

The 32 endoscopic cases had a $91 \%$ success rate with no complications.

Successful patients met the following criteria. They:

- Completed a post-operative evaluation form, rating their result as "successful" or "total cure."

- Reported increase in functional capability.
- Had a negative straight leg raising.

- Are taking no pain medication.

This new endoscope allows inclusion of patients not previously candidates for percutaneous surgery. The automated percutaneous lumbar discectomy procedure has traditionally been an internal decompression which allows subsequent receding of prolapsed disc, not a direct removal of ruptured disc material. This new technique and equipment permits direct removal of ruptured disc as well as internal decompression. As equipment improves for working through small cannulas and more fully eradicating the pathology while also eliminating the trauma inherent in traditional spine surgery, percutaneous techniques are rising in effectiveness while remaining the safest approach.

\section{NEUROSURGERY - VASCULAR $(P-22$ to $P-25)$}

\section{P-22}

Spontaneous Thrombosis of Internal Cerebral Vein: a Case Report

D.F. Louw, K. Reddy, J. Maguire and A. Franchetto (Hamilton, Ontario)

A previously well 29-year-old right handed female developed gradual onset of severe headache the day prior to admission, 2 weeks following an unremarkable cesarean section. Progressive left hemiparesis and drowsiness prompted a neurologic assessment and computed tomography (CT) at another centre. She was afebrile, had a supple neck, reactive pupils, and a left hemiparesis. The original CT demonstrated decreased attenuation of the entire right basal ganglia with midline shift. A repeat $\mathrm{CT}$ revealed in the hypodense area and the development of similar changes on the left side. Mild anemia and hyponatremia were noted with complete normality of all other biochemical, hematologic, and coagulation studies. Her clinical course was rampant with rostrocaudal herniation and brain death occurring within hours of admission despite hyperventilation, mannitol, and ventriculostomy. A review of CT scan findings and the pathological material at autopsy were suggestive of thrombosis of cerebral vein.

\section{P-23}

Intramedullary Injection of Nimodipine into the Spinal Cord Counteracts the Deleterious Effect of Endothelin-1

\section{H. ImAMURA and C.H. TATOR (Toronto, Ontario)}

Intravenously injected nimodipine increases spinal cord blood flow (SCBF) in normal and injured rats. However, in rats with spinal cord injury the hypotension caused by the combination of cord trauma and the vasodilator effects of nimodipine must be counteracted to maintain posttraumatc arterial blood pressure. In contrast, intramedullary injection of nimodipine has the advantage of producing minimal systemic effects. After spinal cord injury blood vessels in the spinal cord show vasospasm, and in the present experiment vasospasm was produced by an intramedullary injection of endothelin-1. With this model we investigated whether nimodipine injected directly into the spinal cord could counteract the vasoconstriction induced by the intramedullary injection of endothelin. 
Male Wistar rats underwent laminectomy at $\mathrm{C} 7$ and $\mathrm{T} 1$. We injected $0.1 \mu \mathrm{l}$ of nimodipine $(0.2 \mathrm{mg} / \mathrm{ml})$, diluent or saline $(\mathrm{n}=$ 5 per group) directly into the spinal cord at C7/T1, and 80 minutes after we injected endothelin- 1 ( 80 pmole in $0.1 \mu \mathrm{l})$ in the same location. SCBF and evoked potentials (EPs: somatosensory and cerebellar evoked potentials) were measured before and after injection of the drugs. Injection of nimodipine, diluent or saline did not cause a significant change in SCBF. Endothelin caused a $60 \%$ decrease $(p<0.05)$ in SCBF 30 minutes after injection in all three groups. In the nimodipine group, SCBF recovered to the baseline value 70 minutes after injection of endothelin. In contrast, there was no significant recovery in the saline or diluent groups. In another group of 5 rats endothelin was injected first, and then $0.2 \mathrm{mg} / \mathrm{ml}$ nimodipine was injected 80 minutes after endothelin. There was a $67.5 \%$ decrease in SCBF ( $<<0.0001$ ), but the SCBF recovered to baseline after the nimodipine injection. Intramedullary injection of endothelin caused systemic hypotension and a significant change in EPs which was not improved by nimodipine. Thus, intramedullary injection of nimodipine counteracted the deleterious effect of endothelin on SCBF but did not improve EPS.

\section{P-24}

Symptomatic Vasospasm Following Clipping of Unruptured Intracranial aneurysms: a Report of 3 Cases

R.L. Sahjpaul, C.G. Drake, R.F. Del Maestro and S.P. LOWNIE (London, Ontario)

Cerebral arterial vasospasm occurring following the clipping of unruptured intracranial aneurysms is an extremely rare event with only nine reported cases. We present three cases of unexpected symptomatic vasospasm following uneventful clipping of unruptured intracranial aneurysms. While the pathogenesis of vasospasm in these instances remains uncertain, current evidence seems to support a hypothalamic mechanism.

\section{P-25}

Awake Craniotomy and Cortical Stimulation Mapping for Resection of Arteriovenous Malformations in/near Eloquent Brain Areas

R.L. Sahjpaul, J.P. Girvin, C.G. Drake, S.J. Peerless and G.G. FERguson (London, Ontario; Miami, Florida)

Arteriovenous malformations (AVM) in/near eloquent brain areas present a formidable therapeutic challenge. Utilizing awake craniotomy and cortical stimulation mapping techniques can allow safer resection of such lesions than would be possible under general anaesthesia, with minimal or no neurologic deficit. The experience over 20 years at this institution is presented.

\section{NEUROLOGY - VASCULAR $(P-26$ to $P$-49)}

\section{P-26}

\section{DNR Determinants in Acute Stroke}

L.T. Smurawska, A.V. Alexandrov, C.F. Bladin and J.W. NORRIS (North York, Ontario)

Background: The issue of maintaining life support systems for many patients with severe stroke is controversial. We previ- ously determined the average cost of in-hospital stroke care in Canada as $\$ 27,000$ per patient, and we have now extended our analysis to evaluate the Do-Not-Resusciatate (DNR) orders in patients with acute stroke and their influence on cost.

Methods: We retrospectively reviewed 150 consecutive stroke deaths. The hospital costs were derived from a computerized database.

Results: Patients who died were predominantly women $(56 \%)$, with a mean age $82 \pm 10$ years, compared to men $(44 \%$, $75 \pm 9$ years). The time to death was the same for both sexes 24 \pm 38 days). There were $68 \%$ patients with cerebral infarcts, and $32 \%$ with intracerebral hemorrhages (ICH). Patients with $\mathrm{ICH}$ died earlier $(9 \pm 10$ days), than patients with infarcts $(30 \pm$ days), ( $p<0.01$ ). DNR orders were given earlier if patient was unconscious on admission ( $2.5 \pm 3$ days). Consultation with family members regarding timing of DNR did not influence time to death. for patients who died within the first week (mean $3 \pm 6$ days) the DNR order was made at a mean $1.5 \pm 8$ days. For patients dying after one week (mean $42 \pm 46$ days) the DNR order was made at a mean $14 \pm 28$ days. Patients who lived less than one week cost $\$ 2,500 \mathrm{CDN}$, whereas those who died after one week cost $\$ 19,000$. Yet, both groups of patients had similar Canadian Neurological Scale scores on admission: $50 \pm 30$ vs. $60 \pm 40$. Using a simple scoring system, systemic complications contributed significantly to the decision for DNR $(p<0.001)$.

Conclusion: Type and severity of stroke and sytemic complications were determinants in DNR orders, but age, sex and the presence of family support were not.

\section{P-27}

\section{Guidelines for Do-Not-Resuscitate Orders in Acute Stroke}

Andrei V. Alexandrov, Patrick M. Pullicino, Eric M. MESLIN and JOHN W. NoRRIS (For the members of the Canadian and Western New York Stroke Consortiums) (Toronto, Ontario; Buffalo, New York)

The Do-Not-Resuscitate (DNR) order withholds cardio-pulmonary resuscitation. There are no disease-specific criteria to aid "no resuscitation" decision-making in patients with acute stroke.

A multicenter Consensus on the Guidelines for DNR Orders in Acute Stroke was developed prospectively at the Canadian and Western New York Stroke Consortiums. A total of 16 Canadian and 10 US neurologists specializing in stroke participated. The clinical criteria for DNR orders were: (1) severe stroke ( $88 \%$ of reviewers), (2) life-threatening brain damage $(73 \%)$, and (3) significant co-morbidities (92\%). The Canadian and US neurologists agreed that the proposed disease-specific Guidelines were appropriate for clinical use (21 of 26 reviewers, or $81 \%$ ). Furthermore, $111 \mathrm{CT}$ scans of patients who died within 4 months following stroke were reviewed to quantify imaging criteria for life-threatening brain damage. Early death following stroke was inevitable if lesion volume was more than $450 \mathrm{ml}$, and midline shift was greater than $6 \mathrm{~mm}$ displacement of the pineal gland.

The disease-specific Guidelines for DNR Orders supplement general DNR policies to aid "no resuscitation" decisions in patients during the first two weeks after stroke who are unlikely to survive the acute phase. The Guidelines are aimed at optimum patient care by preventing unnecessary and futile interventions. 
The International Study of Unruptured Intracranial Aneurysms

JEAN PEACOCK, ISUIA InVESTIGATORs (Rochester, Minnesota)

This study was designed to better define the natural history of unruptured intracranial saccular aneurysms (UIA) to define which subgroups of patients have the greatest risk associated with subsequent aneurysmal rupture and/or surgical repair, to better establish proper management of these patients and to test several hyoptheses about pathophysiological mechanisms underlying aneurysmal development, growth and rupture.

Fifty-three centers will contribute 3500 patients with UIA. Fiteen hundred will be retrospective patients with unoperated UIA seen between 1970 and 1991, half with no history of subarachnoid hemorrhage (SAH) (Group I) and half with a history of SAH (Group 2). Retrospective patients will have a minimum follow-up of 5 years (or until death) from the angiographic diagnosis of UIA. Another 2000 patients will be identified prospectively, approximately half of whom will be unoperated and half will have surgery at the discretion of attending physicians and surgeons. Patients are being followed at 1 month and annually. Arteriograms are all reviewed centrally, utilizing standardized magnification correction techniques. Baseline data have been analyzed for 2000 patients.

The primary analysis will examine fatal and no-fatal intracranial hemorrhagic stroke secondary to aneurysmal rupture. Secondary analyses of neurologic outcome will examine other aneurysmal and surgical complications.

\section{P-29}

\section{Subarachnoid Hemorrhage Presenting as Repetitive TIAs Without Headache}

\section{Z.N. Nasreddine ZIAD and François Grand'Maison (Fleurimon, Sherbrooke, Quebec)}

Subarachnoid hemorrhage (SAH) is classically suspected in patients presenting with severe headaches, nuchal rigidity with or without focal neurological deficits and altered consciousness.

We described a patient with an unusual presentation and discuss possible underlying mechanisms and the danger of misdiagnosis.

A 59-year-old woman known for three episodes of SAH - 25, 15 and 9 years previously - presented with episodic dysarthria and dysphasia, without headache. These episodes occurred twice daily, for three consecutive days. A head CT Scan was compatible with early brain ischemia in a left middle cerebral artery territory. No hemorrhage was apparent. Prior to anticoagulation for presumed repetitive TIAs, angiography showed four newly formed aneurysms and severe vasospasm of the left distal middle cerebral artery. Lumbar puncture revealed xanthochromia with abundant red blood cells.

It was concluded that the repetitive neurological symptoms were caused by the vasospasm secondary to otherwise asymptomatic SAH. The absence of headache could be related to developing siderosis and fibrosis following several episodes of $\mathrm{SAH}$ resulting in relative insensitivity of the underlying meninges and restricted blood spreading. In patients with prior episodes of SAH, rebleeding in the subarachnoid space may present with repetitive focal neurological symptoms without headache.

\section{P-30}

Does a Relationship Exist Between Voluntary Motor Functions and Clinical Spasticity Measured During Sitting in Spactic Hemiparetic Subjects?

\section{Y.K. Kim, C. Hui-ChAN and X. MA (Montreal, Quebec)}

Previously, we found that the reduction in the force generated by maximum isometric voluntary contraction (MVC) of the hemiparetic ankle dorsiflexor during standing, was inversely correlated with the severity of ankle spacticity $(r=-0.65)$ (Levin and Hui-Chan, EMG Clin Neurophysiol 34: 415-425, 1994). The objectives of this study were to investigate the possible relationship between the alterations in voluntary motor functions and clinical spasticity in affected elbow and ankle muscles during sitting, and to establish the test-retest reproducibility of MVC parameters and clinical spasticity in these muscles.

Ten cerebrovascular accident (CVA) patients with chronic spastic hemiparesis and 6 normal, age- and sex-matched subjects performed MVC of biceps, triceps, tibialis anterior and soleus, during which the force and muscle activities produced by these muscles were recorded. The severity of spasticity of both elbow and ankle muscles was determined by a composite spacticity score comprising of 4 and 5 point scales. To examine data reproducibility, all normal and 8 CVA subjects were reevaluated one week later.

Only the voluntary elbow flexion torque was found to be inversely related to the composite spasticity score $(r=-0.70), p$ $=0.05)$ as well as to the upper extremity subscore $(r=-0.82, p$ $=0.01$ ). For all the muscles tested, the peak torque was the most reliable measure (ICC $=0.86$ to 0.99 ) for both subject groups. The MVC peak agonist EMG areas and clinical spasticity score also demonstrated high reproducibility (ICC $=0.64$ to 0.94 and 0.78 , respectively).

Our findings demonstrated that the strength deficit in the spastic elbow flexor was the best indicator of the severity of spasticity during sitting. Furthermore, these MVC data and clinical spasticity score proved to be reliable measurement indexes. We therefore advocate their use in evaluating the effects of treatment over time in hemiparetic subjects.

\section{P-31}

The Aftermath of Stroke: a Need Assessment of Persons With Stroke and Their Caregivers in Nova Scotia

S.J. Phillips, R.F. Lyons and H.I. Stanish (on behalf of the Stroke Work Group of the Heart and Stroke Foundation of Nova Scotia) (Halifax, Nova Scotia)

Stroke is the leading cause of disability and handicap among Canadians. Optimizing the management of the problems encountered by persons who have had a storke remains a challenge for society in general and for the health care system in particular. In order to facilitate the development of a new strategy 
for stroke in Nova Scotia, we preformed a systematic analysis of the perceived needs of stroke survivors and caregivers for rehabilitation and improved quality of life. Participant demographic survey questionnaires and focus group guidelines were developed. Focus group discussions were conducted in six locations in Nova Scotia involving persons who had experienced a stroke $(\mathrm{N}=51$, average age 67 , average time since stroke 5 years), family caregivers ( $\mathrm{N}=41,32$ women) and health professionals $(\mathrm{N}=52)$. The focus group sessions for persons with stroke were designated to be sensitive to cognitive, speech, and mobility problems. Taped transcriptions of the sessions were content analyzed, and the data organized around three areas of illness impact: function, personal identity, and interdependence. It is anticipated that the data gained from this study will assist service providers in Nova Scotia refocus their approach to the complex problems posed by stroke.

\section{P-32}

\section{The International Stroke Trial in Canada}

S.J. Phillips, F. Veloso, A. Hakim and P. Sandercock (on behalf of the International Stroke Trial Collaborative Group) (Halifax, Nova Scotia; Regina, Saskatchewan; Ottawa, Ontario; Edinburgh, UK)

The International Stroke Trial (IST) aims to determine whether antithrombotic therapy for acute ischemic stroke saves lives and reduces disability in survivors. Patients are randomized between a policy of: aspirin $325 \mathrm{mg}$ daily or avoid aspirin; and subcutaneous heparin $(5,000$ or 12,500 units $q 12 \mathrm{~h})$ or avoid heparin. Treatment is continued for two weeks or until discharge from the randomizing hospital. As of 30 November 1994, 8672 patients (45\% over age 75 ) in 29 countries had been randomized. Time from stroke onset to randomization was less than 6 hours in $17 \%, 6-12$ hours in $21 \%, 12-24$ hours in $28 \%$, and $24-$ 48 hours in $34 \%$. During the 14 day treatment period, $4 \%$ of patients experienced another stroke, $2 \%$ had a cerebral or major non-cerebral hemorrhage, and $9 \%$ died. Six months post-stroke, $25 \%$ were dead, and $66 \%$ were dead or dependent. Canada had randomised 36 patients $(0.4 \%$ of total) and has the potential to make a greater contribution to the trial before its completion in mic-1996. The IST has demonstrated that large-scale clinical trials in acute ischemic stroke are feasible. The final results will be widely generalizable to both clinical and public health practice.

\section{P-33}

\section{The Second International Stroke Trial (IST-2)}

S.J. PHILLIPS and P. SANDERCOCK (on behalf of the International Stroke Trial Collaborative Group) (Halifax, Nova Scotia; Edinburgh, UK)

The International Stroke Trial (IST) has demonstrated the feasibility of large-scale interventional studies in acute stroke. It is anticipated that by mid-1996 the trial will have recruited the requisite 20,000 patients to demonstrate statistically significant and clinically important benefits (or hazards) of antithrombotic therapy among clinically identifiable subgroups of patients with acute ischemic stroke. IST-2, currently in the planning phase, will build on the strengths of this experience. Evidence from experimental animals indicates that the volume of brain damaged by ischemia can be reduced by drugs that interfere with the action of glutamate. The aim of IST- 2 is to assess reliably the balance of risks and benefits of treatment with a glutamaterelease inhibitor $(619 \mathrm{C} 89$, Wellcome) among patients within 12 hours after the onset of an acute stroke. Benefit will be measured in terms of reduction in mortality and reduction in disability among survivors. Outcome measures will be developed from those used in the IST. The study involves a unique collaboration between academic physicians and the pharmaceutical industry. We wish to present the protocol to stimulate discussion of design issues in acute stroke trials and to recruit interested investigators.

\section{P-34}

\section{U-shaped Relationship Between Systolic Blood Pressure And Early Death After Acute Ischemic Stroke}

S.J. PHILlips, P. SANDERCOCK and J. Slattery (on behalf of the International Stroke Trial Collaborative Group) (Halifax, Nova Scotia; Edinburgh, UK)

Although blood pressure elevation is common in the setting of acute ischemic stroke, the cause, prognostic implications, and management of this phenomenon are unclear. In the International Stroke Trial of aspirin, heparin, both, or neither for the treatment of acute ischemic stroke, systolic blood pressure is recorded at the time of randomization (baseline SBP). Of the 8672 patients enrolled in the trial by 30 November 1994, 757 (9.5\%) died within 14 days after randomization. Case-fatality was $10.4 \%$ among the 2392 patients with a baseline SBP of 180 $\mathrm{mm} \mathrm{Hg}$ or higher, $7.3 \%$ among the 2257 with baseline SBP in the range $160-179,8.6 \%$ among the 2420 with baseline SBP in the range 140-159, and $12.4 \%$ among the 1603 with a baseline SBP below 140. Outcomes at 6 months (deaths and proportion of patients dead or dependent) showed a similar relation to baseline SBP. These data lead to the testable hypothesis that there is an optimal range for SBP in the first 48 hours after acute ischemic stroke.

\section{P-35}

Stroke Caused by Saccular Aneurysm Thrombosis Without Subarachnoid Hemorrhage

R.D. Brownlee, B. Tranmer and R.J. Sevick (Calgary, Alberta)

A case is presented of a rare cause of ischemic stroke due to spontaneous thrombosis of an unruptured anterior communicating artery aneurysm.

A 66-year-old female presented with acute onset of left hemiparesis. Neurologic examination revealed weakness in the left arm and leg. The remainder of her examination was normal. Laboratory investigations revealed a mild elevation in her partial thromboplastin time and INR and a renal tubular acidosis. Past medical history included alcoholism, chronic obstructive pulmonary disease, chronic renal failure and chronic pancreatitis.

Imaging studies included computed tomographic (CT) scans, magnetic resonance (MR) imaging and cerebral angiography. 
These revealed a thrombosed $1.5 \mathrm{~cm}$ diameter anterior communication (a comm) artery aneurysm with no evidence of subarachnoid hemorrhage (SAH). The right $\mathrm{Al}$ and both $\mathrm{A} 2$ segments of the anterior cerebral arteries were occluded. Cerebral infarction was demonstrated in multiple vascular territories.

The patient died from bronchopneumonia 7 days after her admission to hospital and an autopsy was performed. Pathologic findings included a thrombosed $1.5 \mathrm{~cm}$ diameter anterior communicating artery aneurysm with no evidence of recent or remote $\mathrm{SAH}$. The right $\mathrm{Al}$ and both $\mathrm{A} 2$ segments of the anterior cerebral arteries were occluded with recent thrombus. Cerebral infarcts were found in both anterior cerebral and right middle cerebral artery territories with emboli in small cortical vessels.

This case illustrates a rare complication of an unruptured cerebral aneurysm with imaging and pathologic correlation. Morphologic and hemodynamic features of the aneurysm which may have precipitated thrombosis without rupture are discussed with reference to experimental models.

\section{P-36}

\section{Embolic Lacunar Stroke: Angiographic Demonstration}

Hans MCLelland and Françols GRand'Maison (Sherbrooke, Quebec)

Lacunar stroke is defined as small, deep cerebral infarct in the territory of a single penetrating branch likely due to in situ lipohyalinosis associated with hypertension. However, embolic origin has been suggested as a possible etiologic factor of this stroke but accurate demonstration is lacking.

We present a 63-year-old previously healthy woman with sudden onset of a right pure motor hemiparesis without dysphasia or hemianopia. Head CT-scan was normal. Urgent cerebral angiogram showed a partially-lumen-occluding embolus at the MI-M2 junction of the left middle cerebral artery (MCA) with non-opacification of the lateral lenticulostriates arteries. Embolic workup was negative. She partially recovered and remained with a mild motor deficit in her right hand.

The demonstration of an embolus in the proximal MCA in this patient with a lacunar stroke confirms the embolic hypothesis among etiologies of this type of stroke.

\section{P-37}

\section{The "Three-quarter" Medullary Syndrome}

\section{B.R. Guppy, A.G. Douen and A.BuChan (Ottawa, Ontario)}

Both lateral and medial medullary infarctions have been described in association with vertebral artery occlusions. Medial medullary infarction, often referred to as Dejerine's syndrome, is characterized by an ipsilateral hypoglossal lesion, contralateral hemiplegia and contralateral alteration in deep sensation. Dejerine's syndrome occurs uncommonly and is associated with a Wallenberg's syndrome on rare occasions. A hemimedullary syndrome consisting of an ipsilateral Dejerine's and Wallenberg's syndrome has also been described.

We present a case of a "three-quarter" medullary infarction consisting of a bilateral Dejerine's and a unilateral Wallenberg's syndrome. A 76-year-old man with no history of vascular risk factors or disease presented acutely with vertigo, nausea, quadriparesis. Examination revealed a right Horner's syndrome, jerk nystagmus on right lateral gaze and bilateral tongue weakness. There was a right-sided facial and left-sided body loss of spinothalamic function associated with bilateral impairment of posterior column function. A moderately severe spastic quadriparesis sparing the face was present. MRI and MR angiography confirmed a medullary infarction due to occlusion of the right vertebral artery.

The "three-quarter" medullary syndrome is a unique neuropathological entity which can be detected clinically.

\section{P-38}

\section{Protein Kinase C Activity Changes in Global Ischemic Rats Treated with NBQX and SNX-111}

\author{
F. Wang, B.R. Chakravarthy, H. Li, J.P. Durkin and A.M. \\ BuCHAN (Ottawa, Ontario)
}

We have previously demonstrated that SNX-111, a selective $\mathrm{N}$-type $\mathrm{Ca}^{++}$-channel blocker and an AMPA receptor antagonist, NBQX [2, 3-dihydroxy-6-nitro-7-sulfamoylbenzol $(f)$ quinoxaline], protect $\mathrm{CAI}$ hipppocampal neurons from the effect of 10 min four-vessel occlusion (4VO) in rats. Both drugs offered significant neuroprotection even when the treatment is delayed up to $12 \mathrm{hrs}$ post-reperfusion. Mild intra-ischemic hypothermia, which is neuroprotective, prevented the loss of protein kinase (PKC) activity in the neurons. We have also shown that transcient ischemia induces a rapid (within $1 \mathrm{hr}$ ) $40-50 \%$ reduction in PKC activity in CAl neurons which persists for at least the next 48-72 hrs. We now report the effects of NBQX and SNX111 on post-ischemic PKC activity in CAI neurons. The data indicate that NBQX $(30 \mathrm{mg} / \mathrm{kg} \times 3)$ injected ip immediately upon reperfusion prevented teh loss of PKC activity typically observed 6 hrs after the ischemic insult. However, NBQX (30 $\mathrm{mg} / \mathrm{kg} \times 3$ ) administrated $6 \mathrm{hrs}$ after reperfusion failed to reverse loss of PKC activity at 24 hrs. SNX-111 (5 mg/kg) injected iv 6 hrs after reperfusion also failed to reverse the loss. Since the administration of these drugs at $6 \mathrm{hrs}$ was neuroprotective, it is likely that NBQX and SNX-111 offer neuroprotection by affecting the signaling pathway downstream of $\mathrm{PKC}$ involvement, or alternatively other pathways independent of those which induce PKC inactivation following cerebral ischemia.

\section{P-39}

\section{Vertebro Basilar Blood Flow in "Central" Vertigo}

S.S. Sretenovic, A. Alexandrov, L. Smurawska, D. Brodie and J.W. NORRIS (North York, Ontario)

Vertigo in the elderly is a common symptom associated with changes in head position. However, the role of vertebro-basilar ischemia remains uncertain. To test this, we examined the blood flow velocities (BFV) in the vertebro-basilar arteries in 50 patients with "central vertigo" using Transcranial Doppler (TCD).

12 of the 50 showed decreased BFVs the V-B arterial system (basilar 2, vertebral 4, PCA 3, multiple 3 ) in various head positions. All had clinical or radiological evidence of cervical 
spondylosis. 8 of 12 had reproducible symptoms during maneuvers. Neck extension produced more symptoms than other movements such as flexion and rotation.

Reduced vertebro-basilar BFVs were associated with vertigo in $24 \%$ of cases, who had a greater incidence of severe cervical spondylosis. The commonest precipitating factor was extension of the neck.

\section{P-40}

\section{Carotid Atherosclerosis in Juvenile Diabetics}

L.T. SMURawSKa, D. Daneman and J.W. NorRis (North York, Ontario)

Diabetes mellitus (DM) produces accelerated atherosclerosis with increased risk of vascular complications including stroke. To investigate the impact of vascular disease in diabetics we measured carotid intimal-medial thickness (IMT) and assessed early plaques using high definition ultrasound imaging.

Diabetes is associated with significantly thicker IMT and increased atherosclerotic plaques $(p<0.01)$, compared to normal adult controls. This difference is even greater in insulin dependent (IDDM or Type 1), than non-insulin dependent (NIDDM or Type 2$)$ diabetes $(p<0.01)$. In Type 1 diabetics, there is a further subdivision, since some patients have normal IMT and no plaques, despite long duration of the illness, while others have serious atherosclerosis with much briefer duration of diabetes. To help determine the reasons for this disparity we compared 16 juvenile controls (age $13 \pm 5$ years) to 24 juvenile diabetics (age $12 \pm 3$ years) with duration of diabetes $6 \pm 3$ years. The IMT was thicker in diabetics (common carotid artery $0.051 \mathrm{~cm}$ vs. $0.046, \mathrm{p}<0.05$, and internal carotid artery 0.045 vs. $0.039 \mathrm{~cm}, \mathrm{p}<0.03$ ).

This difference is presumably genetically determined and molecular genetic study is now in progress.

\section{P-41}

\section{Increased p53 Expression in the Neocortex Following} Transient Focal Ischemia

\section{Z.G. Huang, A.M. Buchan, M. Jian and G.S. Robertson} (Ottawa, Ontario)

An apoptotic mechanism may be involved in neuronal death following cerebral ischemia since DNA laddering occurs in our model. P53 expression has been shown to be elevated in damaged neurons that display fragmented DNA following administration of the excitotoxin kainate. It suggests that p53 may contribute to neuronal loss after cerebral ischemia. Present study was designed to address this question by examining changes in cortical p53 mRNA and protein levels following focal cerebral ischemia. Male SHRs were subjected to $90 \mathrm{~min}$ of right middle cerebral artery occlusion, and followed by various times of reperfusion (RP). Six groups $(n=4)$ were sacrificed after $1,4,8$, 12,24 or 48 hrs of RP. Half of the animals in each group were perfused for p53 immunoreactivity. Frozen sections sere obtained from the remaining brains for in situ hybridization histochemical labelling of p53 mRNA. Basal levels of p53 mRNA and protein in the neocortex of sham animals were below the limit of detection. Transient MCA-o produced a dramatic elevation of p53 mRNA in the ischemic region of the neocortex with peak increases occurring 4-8 $\mathrm{h}$ after RP. By comparison, MCA-o had modest effects on $\mathrm{p} 53$ immunoreactivity with maximal elevations detected $12-24 \mathrm{~h}$ after RP. We conclude that the elevation of p53 mRNA and immunoreactivity suggests that this transcriptional regulating factor plays a role in apoptotic death following cerebral ischemia.

\section{$\mathbf{P}-42$}

\section{Cerebellar Infarction: Five Year Experience at the Montreal General Hospital}

Hussain, Al-Rahi, Robert Côté and Dinh Hoang Duong (Montreal, Quebec)

Background: Cerebellar infarction can present with variable clinical features and outcomes. Following recent recent reports we decided to review our experience at the Montreal General Hosptal.

Methods: We retrospectively identified patients admitted to our hospital with cerebellar infarction during a 5 year period (1989-94). Charts were reveiwed for information on vascular risk factors, clinical presentation, investigation, treatment and outcome.

Results: We found 53 patients with a diagnosis of cerebellar infarction. Mean age was 64 y.o., $55 \%$ male and $45 \%$ female. Risk factors included: Hypertension $47 \%$, smoking $42 \%$, heart disease $32 \%$, diabetes mellitus $21 \%$, hyperlipidemia $8 \%$, peripheral vascular disease $6 \%$. Clinical presentation: 19 patients (35\%) had a decreased level of consciousness, most common symptoms were nausea and vomiting $64 \%$, vertigo $58 \%$ and headache $47 \%$. Eight patients had surgery (ventriculostomy). Outcome: 12 patients $(23 \%)$ recovered completely, 19 patients (36\%) had minimal deficits and 7 patients (13\%) remained disabled. Overall, 15 patients ( $28 \%$ ) died, all of which were either comatose or had signs of brainstem extension at presentation.

Conclusion: Outcome in cerebellar infarction is often favorable although decreased level of consciousness at presentation is associated with worse prognosis. The exact role and timing of surgery still has to be better defined for selected patients.

\section{$\mathbf{P - 4 3}$}

\section{Predictive Value of Fibrinogen in Asymptomatic Carotid Disease}

Lucy Vieira, Christina Wolfson, Robert Côté and France BOURQUE (Montreal, Quebec)

Background: Fibrinogen is a known independent risk factor for stroke in population studies. Severe asymptomatic carotid disease also carries an increased risk for cerebrovascular events.

Objective: To assess the value of bifrinogen levels in predicting the degree of carotid stenosis in asymptomatic patients.

Methods: We measured serum fibrinogen levels in patients referred for asymptomatic cervical bruits and correlated these with the degree of carotid stenosis on carotid ultrasonography. Stenosis was reported as percent reduction of lumen diameter.

Results: Eighty-six patients were included in the analysis. 
Mean age was 68.5 y.o. $\pm 9.25,52.3 \%$ were females. Serum fibrinogen level was $3.609 \pm 0.883 \mathrm{~g} / \mathrm{L}$ in 38 patients with $\geq$ $50 \%$ stenosis, and $3.285 \pm 0.698 \mathrm{~g} / \mathrm{L}$ in 48 patients with $<50 \%$ stenosis $(p=0.047)$. Multiple regression analysis for all vascular risk factors showed that only elevated cholesterol was associated with an increased fibrinogen level. The prevalence of elevated cholesterol, however, was not different across the 2 groups.

Conclusion: Serum fibrinogen is a simple and easily accessible hematological test which can identify asymptomatic patients with potentially high grade carotid stenosis. Overall, this could result in an improved investigative process for these high risk patients.

\section{P.44}

\section{Glucose Induced Decrease in Glutamate Levels In Ischemis Human Brain By In-Vivo Microdialysis}

R. Kanthan, A. Shuaib, R. Griebel and H. Miyashita (Saskatoon, Saskatchewan)

Glutamate is one of the principal neurotoxins in the pathogenesis of ischemic neuronal injury. Elevated glutamate levels in ischemia have been well documented in many animal stroke models. Recent work in humans also shows a similar trend. We have used our acute focal ischemic model of the human brain to study the response of glutamate levels by in-vivo microdialysis during ischemia using two different perfusates. The dialysate glutamate levels from probes perfused with Ringer's lactate rose from pre-ischemic values of $33.96 \mathrm{uM} \pm 6.9$ to partial ischemic values of $380.69 \mathrm{uM} \pm 32.4$ reaching a high of 1783 . $\mathrm{uM} \pm$ 277.2 with total ischemia. However, with the addition of $30 \mathrm{mM}$ of glucose to the perfusate the glutamate response was attenuated to $132.17 u M \pm 27.5(\mathrm{P}<.001)$ during partial ischemia and to $664.03 \mathrm{uM} \pm 15.2(\mathrm{P}<0.05)$ with total ischemia. The preischemic values of glutamate remained unchanged. These results indicate that the ischemia-induced rise in glutamate is attenuated by exogenous glucose delivery in the human stroke model.

\section{P-45}

\section{Risk of Hemorrhage from Intracranial Cavernous Malformations}

M.C. Wallace, W. Harper, R. Willinsky, W. Montanera, D. Mikulis, F. Gentill and K. TerBrugge (Toronto, Ontario)

Cavernous malformations with an asymptomatic or symptomatic presentation provide a challenge to the clinician regarding treatment. The natural history of hemorrhage has been reported to be low. The purpose of this retrospective cohort study of largely symptomatic patients was to evaluate the natural history of hemorrhage.

In a multidisciplinary clinic, history was documented with respect to symptoms, seizures and evidence of hemorrhage. Hemorrhage was defined by an obvious neurological deficit and radiographic evidence of bleeding on CT or MR. Cavernous malformations were diagnosed and graded by MR (Zabramski et al.) in 109 cases, and by postoperative pathology in 2 cases. This series included 111 patients harbouring 160 lesions, fol- lowed for a total of 247 patient years (mean follow-up 2-1 years). Of the 111 patients, $73(66 \%)$ had supratentorial lesions and $38(34 \%)$ had lesions in the posterior fossa. Multiple lesions were found in $17(15 \%)$ patients. Lesions in the posterior fossa bled at a rate of $8.2 \%$ per patient per year. Supratentorial lesions bled much less frequently $(0.7 \%$ per patient per year).

Supratentorial cavernous malformations have a benign natural history. Lesions in the posterior fossa have a larger number of clinically apparent events.

\section{P-46}

\section{Delayed Treatment with NBQX or SNX-111 Following} Transient Forebrain Ischemia in Rats

\section{H. LI and A.M. BUCHAN (Ottawa, Ontario)}

Long term neuroprotective effects of glutamate receptor antagonist NBQX and SNX-111 were studied in the 4-vessel occlusion model of transient forebrain ischemia. Adult male Wistar rats were subjected to 10 minutes of forebrain ischemia, followed by delayed treatment by up to $6 \mathrm{~h}$ of a single dose of SNX-111 (5 mg/kg, i.v. bolus infusion), or 3 doses of NBQX (30 mg/kg x 3 i.p., $15 \mathrm{~min}$ apart). Rats were randomised to either 7 or 28 day survival. The injured hippocampal CAl neurons were counted and percentage of total calculated. Statistics employed were the Mann-Whitney $U$ and Kruskal-Wallis tests.

\begin{tabular}{|c|c|c|}
\hline \multicolumn{3}{|c|}{ Duration of reperfusion/ } \\
\hline \multirow[t]{2}{*}{ Groups } & \multicolumn{2}{|c|}{ Percent injured neurons } \\
\hline & 7 days & 28 days \\
\hline saline & $\begin{array}{c}78 \pm 13 \\
n=11\end{array}$ & $\begin{array}{c}80 \pm 19 \\
n=9\end{array}$ \\
\hline SNX-111 & $\begin{aligned} 53 & \pm 30^{*} \\
\mathrm{n} & =11\end{aligned}$ & $\begin{array}{c}80 \pm 12 \\
n=7\end{array}$ \\
\hline NBQX & $\begin{array}{c}45 \pm 33^{*} \\
n=6\end{array}$ & $\begin{array}{l}89 \pm 5 \\
n=4 * p<0.001\end{array}$ \\
\hline
\end{tabular}

These data show that post-ischemic treatment with glutamate antagonists can retard but not prevent the death of CAI neurons following severe forebrain ischemia.

\section{P-47}

The Functional Independence Measure (FIM) in Acute Stroke

\section{J.M. LAWRENCE and S.E. BlaCK (North York, Ontario)}

Introduction: The FIM is a standardized instrument widely used to assess functional disability in stroke rehabilitation, but it has been little used in acute stroke patients.

Method: We prospectively studied 439 consecutive acute stroke patients. The FIM was performed by a nurse, trained to use the scale, in medically stable survivors, at one month, $(\mathrm{N}=$ $248)$, at three months $(N=197)$, and 12 months $(N=230 ; 68$ by telephone FIM).

Results: The disability sub-scales for self-care and ambulation demonstrated at one month and three months respectively: $43 \%$ and $65 \%$ of the patients were independent; $20 \%$ and $15 \%$ 
require minimal and $37 \%$ and $20 \%$ maximal assistance; on the communicative/cognitive sub-scales, $56 \%$ and $71 \%$ were independent; $22 \%$ and $16 \%$ needed minimal and $22 \%$ and $13 \%$ maximal assistance. There was no significant change from three months to one year.

Conclusion: This is one of the first series to use the FIM in an acute stroke sample. The finding that $2 / 3$ of the medically stable survivors recover independence by three months concurs with some previous series, but the FIM allows rating of several different activities, in relation to the amount of assistance needed. We found it to be feasible and believe it is potentially useful in assessing functional outcome and cost effectiveness of stroke interventions.

\section{P.48}

Performance of Non-representational Gestures in Acute Stroke

K.L. Barbour, E.A. Roy, S.E. Black and T.R. Winchester (North York, Waterloo, Ontario)

Objective: To assess the frequency and pattern of impairment in the imitation of non-representational gestures in acute hemispheric stroke. Background: Apraxia is the inability to pantomime or imitate common gestures not related to primary motor or sensory deficits. Previous studies suggest that the testing of non-representational gestures may be more sensitive in assessing apraxia in acute stroke. Method: In a prospective study of 116 acute stroke patients with CT confirmed unilateral lesions [54 right-hemisphere-damage (RHD) and 52 left-hemisphere-damage (LHD)], eight intransitive non-representational gestures were videotaped. Normal limits of performance were determined in 20 age-matched healthy adults. Performance was scored on a 0-2 scale in five dimensions for each gesture including posture of hand, location, action required, plane of movement and orientation of hand. A percent composite scored determined the presence of apraxia. Results: Both groups showed impairment in all performance dimensions except posture of the hand in which LHD patients showed no impairment. The frequency of impairment was significantly higher non-respresentational gestures versus pantomime (79\% LHD and $59 \%$ RHD vs. 54\% LHD and $30 \%$ RHD). Conclusions: Non-representational gestures were impaired in both RHD and LHD patients and appear to be a more sensitive test of praxis. The higher incidence of impairment in non-representational gestural performance presumably arises because the gestural semantic system cannot be utilized to support selection of motor programs.

\section{P-49}

In Vivo Microdialysis Study of Extracellular Glutamate Response to Temperature Variance in Subarachnoid Hemorrhage

R. Kanthan, A. Shuaib, R. Griebel, H. Miyashita and G. GOPLEN (Saskatoon, Saskatchewan)

Neurochemical changes may precede the development of clinical signs in neurological disease. Early identification of such changes may offer an opportunity to avoid or treat complications. Under experimental conditions, extracellular levels of glutamate and other amino acids can be monitored by in-vivo microdialysis in cerebral ischemia, head trauma and epilepsy. Data on the release of glutamate under ischemic conditions in humans is limited. There is no published data on the effects of temperature variation or other manipulations on the extracellular glutamate levels in humans. We report for the first time, the effects of changes in temperature on the extracellular cerebral glutamate levels as measured by in-vivo microdialysis; the dialysate being collected before, during and after cooling in four patients with subarachnoid hemorrhage. Three of the patients had in-vivo microdialysis carried out postoperatively. One patient underwent microdialysis three days prior to the surgical clipping of the aneurysm. In all patients, mild head cooling resulted in a significant decrease in extracellular glutamate levels. The effect of cooling was most apparent when the extracellular glutamate concentrations were high. In two patients, the extracellular glutamate levels increased sharply with fever but returned to normal once the temperature normalized. In-vivo microdialysis can be used to measure extracellular glutamate and other neurotransmitters with minimal discomfort in awake humans. This technique offers a unique opportunity to monitor the neurochemistry in critically ill patients and it may aid in developing therapeutic intervention strategies to minimize undesired chemical responses.

\section{MULTIPLE SCLEROSIS $(P-50$ to $P$-54)}

\section{P-50}

\section{Extrapyramidal Manifestations of Multiple Sclerosis}

S. Boulianne, H. Masson and P. Duguette (Montreal, Quebec) Multiple sclerosis (MS) has a well known protean character. Extra-pyramidal symptoms are unusual, but have been reported. We wish to contribute 3 additional cases of documented MS, two of them harboring abnormal movements as the initial manifestation of their disease.

The first patient had a relapsing-progressive MS with onset in 1974. She had CSF oligoclonal bands. From 1982 through 1987, she harbored a syndrome which consisted of anxiety, frontal features, akathisia and choreiform movements of the four limbs. This resolved despite a deterioration in her global neurological condition. A head CT showed plaques, including one in the right caudate nucleus.

The second patient reported an audible sound in her left ear which was eventually recognized as a palatal myoclonus persisting to this date. She had other manifestations of MS and an MRI showed characteristic diffuse plaques. We have found no other case of a palatal myoclonus as the initial symptom.

The third patient was referred at age 35 for episodic abnormal movements which were recognized as paroxystic kinesigenic dystonic movements which responded to carbamazepine. The head MRI showed diffuse demyelinating plaques. MS is recognized as one of the most frequent etiologies of that syndrome in middle-aged adults. 
Empiric Recurrence Risks for Biological Versus Nonbiological Children of Multiple Sclerosis (MS) Patients

\section{S.A. WARREN and K.G. WARREN (Edmonton, Alberta)}

Previous research has shown an excess risk of MS among children of MS patients (not separating biological from nonbiological children), that supports a genetic contribution to the disease. This study examined the recurrence risks for biological versus nonbiological (step/adopted) children of MS patients.

A mail survey of all patients registered with the University of Alberta MS Clinic (Edmonton, Canada) was supplemented by a file search. The clinic neurologist reviewed clinical material or assessed the children of index patients before accepting them as having MS.

The study population consisted of 530 female and 253 male patients. Data was available on gender and disease status for 1103 biological children (520 daughters/583 sons) and 121 nonbiological children ( 56 daughters $/ 65$ sons). 11 biological children had MS ( 7 daughters/4 sons), and 1 adopted daughter. The crude empiric recurrence risks $( \pm 95 \%$ confidence interval) for children of MS patients were: biological daughters $1.35 \%$ ( \pm $.51)$; biological sons $.69 \%( \pm .34)$; nonbiological daughters $1.79 \%$ ( \pm 1.77$)$; nonbiological sons -

Research with larger numbers of nonbiological children needs to be conducted; however, if the similarity in risk for MS patients' biological versus nonbiological children were confirmed, this would support the importance of an environmental trigger to the disease.

\section{P-52}

Immunoglobulin Heavy Chain Variable Region Polymorphisms and Multiple Sclerosis Susceptibility

ROBERT B. BELL, C.E. MowAT and E.C. TwIST (Calgary, Alberta)

Multiple sclerosis (MS) is considered a complex genetic disorder, where no discernable pattern of inheritance is evident. Epidemiological studies suggest a polygenic or multi locus model for susceptibility and this is supported by the polygenic nature of experimental models of autoimmune disease. In MS only two genetic loci have reproducibly been identified to influence disease susceptibility. The first of these lies within the MHC complex, but no specific allele or allele sequence has yet been identified which is mandatory for the development of disease. The second of these lies within the immunoglobulin heavy chain variable region (IGVH) as demonstrated by prior association studies, however the specific nature of the genetic sequence variation responsible for this susceptibility has not been identified.

Using both affected sibling pairs and non familial MS patients in linkage and association studies, we have examined the IGVH region further. Utilitzing repetitive sequence and single base pair polymorphisms obtained by systematically sequencing across the IGVH region, we have defined the genomic limits of association and have identified the polymorphic sites that have the highest association with disease within these limits. All relative risks associated with marker genotypes have been examined in both DR2+ ${ }^{+}$and DR2- populations.

\section{Close Onset of Pseudotumor Cerebri and Multiple Sclerosis:} Causal Relationship or Coincidence?

Ziad Nasreddine, François Evoy and Jean-Pierre Brenier (Sherbrooke, Quebec)

Pseudotumor Cerebri (PTC) is a clinical syndrome with many known etiopathogenic factors. We describe one patient with which PTC, and probable multiple sclerosis (MS), both appeared over a short period of time. We did not find any described association between those two conditions.

A 43-year-old woman, presented with transient visual obscurations and blurred vision, over a 5 month period. She experienced no headache. Neurologic examination revealed bilateral papillary edema with no other abnormalities. A brain CT Scan was normal and two lumbar punctures showed increased CSF pressure $\left(30 \mathrm{~cm} \mathrm{H}_{2} \mathrm{O}\right)$, and the presence of oligoclonal bands. Visual evoked responses were normal. Six months after the onset of her symptoms, she had a sudden onset of sensory loss in the lower extremities, and examination was compatible with myelitis with a sensory level at $T_{10}$. Signs and symptoms disappeared after 3 weeks. MRI of the brain and spine was compatible with MS. PTC and MS like picture could both be caused by lupus, antiphospholipid syndrome, sarcoidosis, and Behcet disease. These disorders are excluded at the time of the present evaluation.

Further follow-up will be required to help elucidate this peculiar association between PTC and probable MS.

\section{P-54}

A Comparison of Intravenous Versus Intrathecal Administration of MBP Synthetic Peptides in Chronic Progressive Multiple Sclerosis

\section{K.G. WARREN and INGRID CATZ (Edmonton, Alberta)}

Subsequent to purification of anti-MBP from cerebrospinal fluid (CSF) and brain tissue of multiple sclerosis (MS) patients, the MBP epitope for anti-MBP has been localized to an area between Pro 85 and Pro 96. This B cell response is shared with the immunodominant $T$ cell response which was shown to be directed to MBP residues 86-99. MBP synthetic peptides containing this epitope have been administered intravenously and intrathecally to MS patients with chronic progressive disease. Intrathecal administration of peptides MBP 75-95 and MBP 8695 at weekly intervals for periods up to 10 weeks produced complete binding of free (F) anti-MBP and had no effect on titers of bound (B) antibody. In comparison a single intravenous injection of these same MBP synthetic peptides resulted in neutralization of both F and B anti-MBP levels in CSF. These results suggest that intrathecal administration of synthetic peptides containing the MBP epitope blocked $\mathrm{F}$ anti-MBP whereas intravenous administration of these peptides downregulated their synthesis. Kinetic curves of intrathecal and intravenous administration of MBP synthetic peptides will be presented. 


\section{NEURORADIOLOGY $(P-55$ to $P$-59)}

\section{P-55}

Serial rCBF SPECT: a Potential Tool for the Diagnosis of Alzheimer Disease and Depression

L.J. Kremer, Haim Golan, Joel Kirsh, Masanori ICHISE and MoRris Freedman (Toronto, Ontario)

Although Alzheimer's disease (AD) and depression (DEP) represent separate pathophysiological processes, it may be difficult to distinguish early AD from DEP on clinical grounds alone. SPECT has been shown to be useful for the differential diagnosis of these disorders. Here, we postulated that serial SPECT may provide additional diagnostic information by showing direction of change in perfusion over time, i.e., patients with $A D$ should show progressive worsening on SPECT whereas patients with DEP should show improvement.

Thirteen patients with AD (73 \pm 9 years) an 7 with DEP (56 \pm 14 years) were referred from the Behavioural Neurology Program at Baycrest for Tc-99m-HMPAO SPECT. Only patients who had at least 2 scans within a 36 month period were included. The radiologists were blinded to the clinical information. Findings were categorized on the basis of visual analysis into three patterns: worsened, improved, unchanged. Results showed:

\begin{tabular}{lccc} 
& Worsened & Improved & Unchanged \\
\hline $\mathrm{AD}$ & $11(85 \%)$ & 0 & $2(15 \%)$ \\
$\mathrm{DEP}$ & $1(14 \%)$ & $4(57 \%)$ & $2(29 \%)$ \\
\hline
\end{tabular}

Our findings suggest that serial SPECT scanning may differentiate between $A D$ and $D E P(P<0.05)$. When the differential diagnosis lies between $A D$ and $D E P$, worsening of rCBF strongly supports a diagnosis of AD. Moreover, improved $\mathrm{rCBF}$ argues strongly against $A D$ and in favor of DEP. Prospective studies to confirm our findings are warranted.

\section{P-56}

Fatal Reaction From Inadvertent Intrathecal Entry of an Ionic Contrast Medium (Hypaque)

\section{R. SAHJPAUL, D.H. LeE and D.G. Munoz (London, Ontario)}

Inadvertent intrathecal administration of ionic contrast can have catastrophic consequences. Accidental intrathecal administration during epidurography, incorrect product labelling, and physician error have been reported as causes for such mishaps. We report a case of a patient undergoing a nephrogram with an ionic contrast medium (Hypaque) who developed neurologic complications and subsequently died following the procedure. At autopsy, a pathological fistulous connection between the renal pelvis and the thecal sac was demonstrated. To our knowledge, such an occurrence has not previsouly been reported.

\section{P-57}

Superior Sagittal Sinus Involvement By Parasagittal Meningiomas: Comparison of Angiography and MRI

Michael Tymianski, W. Morrish, B. Willinsky, W. Montanera, K. TerBrugge, A. Guha and D. Mikulis (Toronto, Ontario)
Purpose: to compare angiography and MRI in assessing superior sagittal sinus (SSS) involvement in parasagittal meningiomas. Methods: 20 patients with parasagittal tumors (19 meningiomas, 1 plasmacytoma) were blindly retrospectively reviewed. All had conventional angiography and MRI prior to surgical resection. Surgical findings, as reported in the surgical notes, were used as the gold standard. Results: Conventional angiography accurately assessed SSS invasion in 70\% of cases and SSS patency in $91 \%$. MRI accurately assessed SSS invasion in $80 \%$ of cases and SSS patency in $84 \%$. These findings showed no statistically significant difference using Fisher's exact method (sinus invasion $p=.16$, sinus patency $p=.23$ ). Concordant angiographic observations were recorded in $65 \%$ of cases assessing SSS invasion, and $85 \%$ assessing SSS patency. Intraobserver concordance was $70 \%$ for sinus invasion and $80 \%$ for sinus patency with MRI. Conclusion: No significant difference was found in the ability of angiography and MRI to assess SSS invasion and patency in parasagittal meningiomas.

\section{P-58}

\section{Reversible Morphological Brainstem Distortion as Visualized on MRI}

\section{S. Casha, D. Mikulis and A.Guha (Toronto, Ontario)}

We describe five patients who presented with headache and evidence of brainstem dysfunction. They were found on imaging to have small bilateral subdural collections and obliteration of the basal cisterns, which were felt not to account fully for the extent of their neurological symptoms. However, MRI revealed gross elongation of the anterior-posterior dimension of the brainstem and, in three patients, descent of the cerebellar tonsils into the foramen magnum. These radiological findings which correlated with the clinical findings, were not visualized on initial routine CT imaging. The MRI findings could be explained in two ways. In three patients, the small but bihemispheric subdural collections could have caused downward displacement of brainstem due to mass effect or venous congestion. In two patients however, the primary insult was decreased pressure below the foramen magnum. This may have caused brainstem distortion by a mechanism similar to Acquired Chiari Malformation. Management of the five cases was directed at the proposed underlying etiology. Patients with the bilateral small subdural collections alone, had resolution of their clinical symptoms by surgical evacuation, with concomittant resolution of their MRI abnormalities. In the remaining patients, treatment directed at correction of the low spinal CSF pressure achieved similar symptomatic and radiological resolution. These cases illustrated the usefulness of MRI in patients with clinical evidence of brainstem dysfunction not accounted for by the pathology seen on routine CT imaging.

\section{P-59}

An Assessment of Regional Atrophy and Cerebral Blood Flow Utilizing HmPAO SPECT and CT Scanning for the Identification of Alzheimer's Disease (AD)

Howard Feldman, Andre Mattman, Michael Schulzer, Bruce Forster, David Li, Walter amman and Lynn Beattie (Vancouver, British Columbia)

Objectives: To evaluate the utility of combining semiquantitative HmPAO SPECT scanning with both qualitative and 
quantitative regional CT atrophy measurements to distinguish AD from not demented (ND).

Background: A variety of approaches have been proposed to enhance the accuracy of HmPAO SPECT scanning for AD diagnosis. Combining regional cerebral blood flow SPECT data with corresponding regional $\mathrm{CT}$ atrophy measurements may improve diagnostic ability.

Methods: A total of 122 referred subjects to the UBC Alzheimer Clinic with a diagnosis of "probable $A D$ " $(N=49)$, "possible" AD $(\mathrm{N}=36)$ and ND $(\mathrm{N}=37)$ were included. The variables for SPECT analysis were 6 regional perfusion ratios and for CT 12 qualitative and quantitative measures of cerebral atrophy. Discriminant analysis was performed on the combined data and ROC curves were applied to test the accuracy of the derived measures to distinguish AD from ND.

Results: Discriminant analysis identified CT left cortical temporal atrophy and SPECT left temporal perfusion as the most important variables in distinguishing AD from $N D(p<$ 0.05 ). The ROC curves based on the discriminant function had an area of $80 \%$ (SE 5\%), compared to $74 \%$ (SE 5\%) for CT or to $70 \%$ (SE 5\%) for SPECT.

Conclusion: The combination of SPECT perfusion ratios and CT atrophy scores did not significantly improve upon the ability of SPECT alone $(P=.16)$ or CT alone $(P=.40)$ alone to correctly identify AD patients.

\section{PERIPHERAL NERVE $(P$-60 to $P$-69)}

P.60

\section{Diagnostic Difficulties in Myasthenia Gravis}

John Maher, Charles Bolton, François Grand'Maison and Michael Strong (Winnipeg, Manitoba; London, Ontario; Sherbrooke, Quebec)

Background: The diagnosis of myasthenia gravis is based on clinical, electrophysiological and immunological findings, and a Tensilon test. We describe four patients whose findings were misleading. Methods: Patients had routine electrophysiological studies, including repetitive nerve stimulation, phrenic nerve conduction studies, and needle electromyography of the diaphragm. Results: All patients had typical fluctuant muscle weakness and positive Tensilon tests. However, weakness predominated in bulbar and respiratory muscles. On initial testing, $\mathrm{AChR}-\mathrm{Ab}$ titres were negative, and only two patients had a greater than $10 \%$ decremental responses with repetitive nerve stimulation. All had very low amplitude diaphragmatic compound muscle action potentials, and fibrillation potentials and small, brief polyphasic motor units on needle electromyography of the diaphragm. Electrophysiological studies were otherwise unremarkable. Clinical and Electrophysiological findings improved with treatment for myasthenia gravis, which included corticosteroids. Subsequent testing showed typical decremental responses in all patients and one had a weakly positive $\mathrm{AChR}$ Ab titre. Conclusion: We suggest that electrophysiological findings may be misleading in myasthenia gravis, particularly when involvement is predominantly respiratory and $\mathrm{AChR}-\mathrm{Ab}$ titres are negative. Careful consideration of clinical symptomatology, a Tensilon test, and a trial of immunosuppression may be necessary to establish the diagnosis.

\section{P-61}

\section{Dropped Head Syndrome with Dystrophic Findings on Muscle Biopsy}

ANDREW KIRK and Robert J.B. MaCaulay (Saskatoon, Saskatchewan)

Weakness restricted to neck extensors is an uncommon clinical presentation and several pathologic correlates have been reported. We describe a 57-year-old man who presented in May, 1993 with one year of marked neck extensor weakness to the point where he could not maintain his head erect for more than a few seconds at a time. The only other finding was minimal weakness of neck flexion and shoulder abduction bilaterally. CK level was slightly elevated. EMG, including neck extensors, was normal. Edrophonium test and anti-acetylcholine receptor antibodies were negative. A trial of oral prednisone was unhelpful. Trapezius biopsy in 1993 showed scattered rounded atrophic fibers with no inflammation. Biopsy of a deep neck extensor in October, 1994 showed interstitial fibrosis, myofiber atrophy, degeneration, regeneration, and hypertrophy, and ring fibers compatible with a dystrophy. Inflammatory infiltrates characteristic of facioscapulohumeral dystrophy were absent. No ubiquitin positive inclusions were seen and there was no ultrastructural evidence of inclusion body myositis. Almost three years into his illness, the patient's condition remains the same with no further generalization of weakness. Dropped head syndrome has several possible causes, one of which appears to be a localized dystrophy.

\section{P-62}

Cardiac Abnormalities Associated with Inclusion Body Myositis

C.A.J. Boyle, C. Wells, D.Gross, B. Cujec and J.R. Donat (Saskatoon, Saskatchewan)

Polymyositis, dermatomyositis and inclusion body myositis are acquired inflammatory myopathies. Cardiac abnormalities have been reported with polymyositis and dermatomyositis, but have not been well documented in inclusion body myositis. Six patients with biopsy-proven inclusion body myositis were assessed for the presence of cardiac disease. The patients had typical symptoms, clinical course, EMG and biopsy findings of inclusion body myositis. Investigations included electrocardiogram, echocardiogram, coronary angiogram, and cardiac biopsy. Five had cardiac risk factors. Two had coronary artery disease. One of the patients was diagnosed as having inflammatory myopathy, when she had persistant elevation of CK following a myocardial infarction. Five had cardiac conduction abnormalities, with two requiring pacemakers. Two patients had cardiomyopathy. One of the patients with cardiomyopathy had a dramatic improvement in his ejection fraction following a course of immune globulin. Both patients with cardiomyopathy had cardiac biopsies, both of which were negative for the characteristic histologic and ultrastructural changes of inclusion body myositis. More observations, especially in patients without confounding factors, and further clinicopathologic correlation are required to clarify causality. In this series of patients there was a high incidence of cardiac disease, particularly cardiac conduction 
abnormalities and cardiomyopathy, suggesting that there is a strong association between these abnormalities and inclusion body myositis.

\section{P-63}

\section{Motor Neuron Disease and Monoclonal Gammopathy}

\section{MoniQue D'Amour (Montreal, Quebec)}

In the literature, motor neuron disease has been discribed in association with monoclonal gammopathy. Such cases have lower motor neuron signs and may have a few upper motor neuron signs. However, there is no precise evaluation of these signs under the operating criteria suggested by the World Federation of Neurology for a diagnosis of Amyotrophic Lateral Sclerosis.

We present two patients with lower motor neuron disease and monoclonal gammopathy. Their clinical, electrophysiological, biological and radiologic findings are described. There is particular involvement of the upper limbs. Progression is slow, with periods of exacerbation. One patient has few bulbar signs. Response to plasma exchange is discussed.

However, these cases did not meet the specific criteria for Amyotrophic Lateral Sclerosis as described by the World Federation of Neurology. We suggest therefore that lower motor neuron disease in association with monoclonal gammopathy represents a different entity.

\section{P-64}

Miller Fisher Syndrome, Campylobacter, and Anti-GQ1B Antibodies

\section{A.-L. Lafontaine, F.P. Thomas and C.H. Chalk (Montreal, Quebec)}

Recently, there has been interest in the relationship between Guillain-Barré syndrome (GBS), campylobacter, and antiganglioside antibodies. In typical GBS, preceding campylobacter infection is common, but the status of antiganglioside antibodies is unclear. Conversely, in the GBS variant Miller Fisher syndrome, there is a close correlation with anti-GQ1B antibodies, but an association with campylobacter has only rarely been reported.

A 25-year-old man presented with six weeks of diarrhea and 10 days of bilateral ptosis, diplopia, and dysphonia. He had begun working in a slaughterhouse three months before. Complete ophthalmoplegia, ptosis, hyporeflexia, and mild gait ataxia were present. CSF was acellular with protein $1.3 \mathrm{~g} / \mathrm{L}$. Median and sural sensory responses were absent but motor conduction was normal. Stool cultures grew Campylobacter coli. Serum anti-GQ1B antibodies were present (titre 1:200; 1:100 five weeks later). Five days after admission he developed a right facial palsy. No specific treatment was given, and he recovered completely in three months.

We suspect that the association of campylobacter infection and anti-GQ1B antibodies seen in this case of Miller Fisher syndrome is not rare, and that it has a fundamental role in pathogenesis. Immunohistochemical studies of our patient's serum are in progress.

\section{P-65}

\section{Induction of Interleukin-6 in Sensory Neurons After Nerve} Injury

P.G. Murphy, M. Altares, J. Grondin and P.M. Richardson (Montreal, Quebec)

An inflammatory response in a dorsal root ganglion has been shown previously to be beneficial to axonal regeneration. In a search for cytokines that might mediate this action, the concentration of interleukin-6 mRNA in lumbar dorsal root ganglia was found to be markedly increased from 1-14 days after nerve injury. The induction of interleukin mRNA was documented by reverse transcription polymerase chain reaction and by ribonuclease protection assay, and was associated with the presence of bioactive interleukin-6 in the ganglia. Unexpectedly, interleukin6 mRNA was localized by in situ hybridization to a subpopulation of large and medium-sized sensory neurons. Thus, neurons like several other cell types express the interleukin- 6 gene strongly but transiently after injury. The possible autocrine or paracrine actions of interleukin- 6 mRNA in dorsal root ganglia are unknown.

\section{P-66}

\section{Cosegregation of Hip Dysplasia and Charcot-Marie-Tooth Disease in One Family}

Stéphane Charest, F. Grand'Maison and R. Dumais (Sherbrooke, Quebec)

Hip dysplasia (HD) has been observed sporadically in subjects with Charcot-Marie-Tooth (CMT) disease. Familial clustering of HD has not been reported. We present a family in which five members were affected by both conditions.

Seven members of a family of nine - spanning over three generations - were assessed. The diagnosis of CMT required electrophysiological confirmation. The diagnosis of HD was based on radiological criteria.

On radiological investigation, HD was confirmed in four individuals known for hip disease since early adolescence and HD was detected in one asymptomatic young adult Neurological evaluation revealed signs of a chronic polyneuropathy in these five family members. Nerve conduction studies were consistent with CMT type 1 .

In the other two family members, neurological and orthopaedic evaluations were normal and investigation failed to disclose subclinical evidence of CMT or HD.

The association of CMT and HD in this family is striking and may result from the cosegregation of closely linked genetic abnormalities. Molecular analysis may therefore reveal a novel genetic abnormality responsible for CMT type 1 . 
Ulnar Neuropathy as a Complication of Spasmodic Torticollis

\author{
C.A. De Meulemeester, C.L. Barclay and P.R. Bourque \\ (Ottawa, Ontario)
}

Most ulnar neuropathies at the elbow result from entrapment in the cubital tunnel or condylar groove. In this report, we illustrate how sustained elbow flexion by itself may injure the ulnar nerve. We also document a very unusual complication of spasmodic torticollis.

The patient, a 54-year-old woman, was treated with Botulinum toxin for spasmodic torticollis and subsequently developed marked neck weakness. In order to support her head, she continuously held it with her right hand, necessitating full flexion of the elbow. One week later, she complained of numbness in the distribution of the right ulnar nerve. The following month, she showed atrophy and weakness of ulnar innervated right hand intrinsic muscles. There was severe hypaesthesia in the distribution of the palmar, dorsal, and digital branches of the ulnar nerve. ECG/NCS showed an isolated severe ulnar neuropathy with axonal loss and only minimal slowing of motor conduction velocity at the elbow.

Prolonged elbow flexion has been implicated in the pathogenesis of ulnar neuropathy, mostly in settings where concurrent external compression is likely: anesthesia, coma. We postulate that sustained stretching from elbow flexion may be sufficient to cause axonotmesis.

\section{P-68}

\section{Levels of ras-GTP in Operative Specimens From NF-1 and Non NF-1 Related Peripheral Nerve Tumors}

A. Guha, N. Lau, G. Boss and A. Pawson (Toronto, Ontario; San Diego, California)

Neurofibromin, the normal gene product of NF-1, acts as a tumor suppressor by blocking ras mediated proliferation via two possible mechanisms. Through its GTP'ase Acting Protein like domain it can be a upstream inactivator of ras-GTP, and/or a downstream negative effector upon binding to activated rasGTP. In neurogenic sarcoma cell lines increased ras-GTP levels, measured by $\mathrm{p}^{32}$ labelling, supports the upstream model. Whether ras-GTP levels are elevated in neurogenic sarcoma or neurobibroma specimens from NF-1 patients, and how do these levels compare to schwannomas or traumatic neuromas from non NF-1 patients are not known, and was measured in this study.

A non-radioactive quantitative colorimetric luciferase based assay for ras-GTP using NDP Kinase, was developed for cell lines and adapted for tissues. Flash frozen operative specimens were analyzed for ras-GTP levels and standardized per $\mu \mathrm{g}$ of DNA, to reflect the amount of activated ras in the tumor cells. The neurogenic sarcomas from the NF-1 patients had the highest levels of activated ras-GTP followed by the neurofibromas. The levels of ras-GTP were low and not different between schwannomas and traumatic neuromas from non NF-1 patients. Levels of neurofibromin protein and $\mathrm{mRNA}$ are being determined in the specimens using immunoprecipitations and RT-PCR respectively.
Our data supports the upstream model of neurofibromin leading to tumor genesis in NF-1, though it does not preclude a concomitant inhibitory downstream ras effector function.

\section{P-69}

\section{Three Weeks Paralysis After Long-Term Vecuronium} Administration

D.R. Mclean, A. Al-Sanei, M. Barbary, S. Mousa, B. STIGSBY, M.Z. Al-KaWı and S. BoHLEGA (Riyadh, Saudi Arabia)

With increasing use of neuromuscular blockade in Intensive Care Units, prolonged blockade after long-term vecuronium administration is being recognized. We report a 60 -year-old woman who had continuous vecuronium for four days following coronary by-pass surgery. Acute tubular necrosis developed post-operatively. After stopping vecuronium she remained completely paralyzed with no evocable eye movements and absent corneal, gag and tendon reflexes. Her pupils reacted sluggishly. Persistent neuromuscular paralysis was confirmed by the absence of any motor response on maximal ulnar nerve stimulation. Gradual return of strength occurred over 21 days. Her renal function was impaired during the time of paralysis. Repetitive nerve stimulation showed a marked decrement on repetitive stimulation. Prolonged vecuronium blockade usually occurs with impaired renal failure. Prolonged blockade following longterm vecuronium needs to be considered when persistent weakness is encountered following the use of this drug.

\section{MOVEMENT DISORDERS $(P-70$ to $P$-77)}

\section{P-70}

\section{Therapeutic Effects of Flunitrazepam in Patients With} Dystonia

Reina Benabou, Raul Marino Jr., Salomon Benabou and Pedro Molina Negro (Montreal, Quebec; Sáo Paulo, Brazil)

Flunitrazepam (FN) is a powerful agonist of all benzodiazepine receptors of GABAergic neurons. We found it effective for the treatment of dystonia especially those in whom the hypnotic effect is absent or low. 15 patients with primary dystonia were studied over two years, using the Fahn and Marsden Movement and Disability scale and video-recording. Daily dose varied from 4 to $40 \mathrm{mg}$. We characterized two distinct groups of patients with different response patterns. There was a clear inverse correlation between hypnotic and antidystonic effect: 11 patients, who had the best improvement rates (54 to $100 \%$ ) did not show daytime drowsiness and four patients who showed excessive day drowsiness had lower improvement rates (32 to $50 \%$ ). FN has shown a specific therapeutic effect in dystonic patients independent of its hypnotic action. Based on the fact that there are different types of benzodiazepine receptors, and since dystonia is a genetically determined disorder, we suggest that dystonic syndromes may be due to different genetically determined benzodiazepine receptor dysfunction. It may be possible to identify different subgroups of dystonia according to the therapeutic response. 
Parkinson's Disease Diagnosis by History - Significance to Genetic Studies

A.H. Rajput, M. Fenton, W. Wilson, L. McCulloch, M. SINKE and B. KANIGAN (Saskatoon, Saskatchewan)

By the time Parkinson's disease (PD) manifests, the parents are usually deceased, children are too young to have PD, and other siblings are scattered widely and are inaccessible for examination. Most studies of PD families therefore rely on history for secondary cases.

We report our observations in patients seen at the Movement Disorder Clinic (MDC). All movement disorder (MD) cases identified through the proband, those seen independently but were related to the proband, and those deceased, but reported as having "similar" problem and had autopsy studies, were included. The 32 PD probands had 43 secondary MD cases - 35 (81.3\%) $\mathrm{PD}$ and 7 (16\%) essential tremor (ET). In three families only ET and in three other families both PD and ET cases were identified. Autopsy was conducted in 11. In one large PD family, a "similar" condition was reported in one deceased member who had normal examination of brain.

These observations indicate that history (alone) suggesting $\mathrm{PD}$, has major limitations for the diagnosis of secondary cases. Tremor and age-related slowing, erroneously diagnosed as PD, confound the interpretation of genetic studies.

\section{P-72}

\section{Measurement of Rigidity in Parkinson's Disease}

J.H. Jhamandas, A. Prochazka, D.J. Bennett, M.J. Stephens, R. SEARS-DURU and T. RoBERTS (Edmonton, Alberta)

Clinical assessment of rigidity in parkinsonian patients is largely qualitative. The reliability and validity of the assessments is sometimes in doubt. Several "engineering" methods of quantifying rigidity have been described, but none has been adopted in general clinical practice. A possible reason is that these methods differ in crucial aspects from the clinical exam. We therefore tackled the problem by monitoring the clinical exam itself, using small sensors to measure the forces and displacements applied. Limb stiffness was computed using parameter identification methods and compared to clinicians' verbalized ratings of rigidity based on a five point scale: United Parkinson's Disease Rating System (UPDRS). The qualitative and quantitative estimates co-varied over a surprisingly large range, depending on the forces imposed and the subject's motor set. Clinicians differ by up to one full point in their mean qualitative ratings and sometimes disagreed on whether levodopa reduced rigidity. This was not due to systematic differences in the overall range of rigidity they evoked, but rather to the way they scored this range. The importance attached to minimal, mean and maximal stiffness evidently differed. Our results indicate that the current clinical exam may be too abbreviated to detect the sometimes quite small reductions in rigidity after levodopa. Our goal is to further develop our system to provide a standardized device for quantifying parkinsonian rigidity.

\section{Restoration of Nigro-Striatal Circuitry by Simultaneous Embryonic Striatal and Nigral Grafts}

\section{Ivar Mendez and Renn Holness (Halifax, Nova Scotia)}

Evidence for survival and growth of fetal nigral grafts when implanted in the adult host striatum is well documented. However attempts to restore the nigro-striatal circuitry in the ventral mesencephalon have been unsuccessful so far. We have employed a novel micrografting technique to implant embryonic striatal and nigral cell suspensions in the rat model for Parkinson's disease. Fetal striatal and nigral cell suspensions were stereotactically implanted in two different sites in the ventral mesencephalon of rats. The animals had received previously Ibotenic acid and 6Hydroxydopamine lesions in the ipsilateral substantia nigra. Animal locomotor activity was assessed pre and postgrafting using a video activity monitor. This study identifies the neurotransmitter profiles of nigral dopaminergic cells using an antibody against thyrosine hydroxylase (TH) and of striatal GABAergic cells using an antibody against glutamic acid decarboxylase (GAD). Dopaminergic axons arising from the nigral graft extensively reinnervated the striatal graft, in this way recreating a small nigro-striatal circuitry in the ventral mesencephalon. Grafted animals showed recovery in locomotor activity three months after grafting.

This study demonstrates for the first time the formation of a nigro-striatal circuitry by simultaneous embryonic striatal and nigral grafts in the ventral mesencephalon of lesioned rats.

\section{P-74}

In Vivo Binding of the Dopamine D1 Receptor Antagonist (C-11) SCH39166 in MPTP-treated Rhesus Monkeys by Positron Emission Tomography (PET)

D.J. Doudet, G. Chan, T. Dobko, E. Sturm, S. Jivan, D.B. CALNE and T. RUTH (Vancouver, British Columbia)

Using PET, we investigated in vivo, the binding of (C-11) SCH39166, a selective DA DI receptor ligand, in the MPTPinduced model of Parkinson's disease in rhesus monkeys.

Five monkeys with MPTP-induced unilateral nigro-striatal lesions (as assessed by FDOPA scans) and five controls were scanned with 3 - $5 \mathrm{mCi}$ of SCH39166 in an ECAT 953B-31. Four lesioned monkeys were asymptomatic and one had profound impairments. The ratios of the striata (caudate and putamen) to a non-specific area (cerebellum) $30-45$ min post injection were analyzed with paired and unpaired $t$-tests.

D1 receptor binding was significantly decreased in both the ipsi and contralateral striata of the unilaterally MPTP-treated animals. The binding in the MPTP-affected striatum of the four asymptomatic animals was significantly decreased compared to the contralateral striatum while it was increased in the severely impaired animal.

The role of D1 receptors in extrapyramidal function is not completely understood. The location of the D1 receptors in the basal ganglia is uncertain. The decreased binding may represent a compensatory mechanism to the loss of DA in both striata since postmortem cell counts have shown that the striatum contralateral to MPTP injection usually also sustains some degree of damage. 
In Vivo Binding of (C-11) Raclopride in Asymptomatic and Symptomatic MPTP-Treated Rhesus Monkeys by PET

D.J. Doudet, G. Chan, T. Dobko, E. Sturm, J. Huser, D.B. CALNE and T. RUTH (Vancouver, British Columbia)

Upregulation of the striatal dopamine D2 receptors has been reported in Parkinson's disease. We investigated in vivo by PET the fate of the dopaminergic D2 receptors in rhesus monkeys with varying degrees of striatal dopaminergic loss.

Seven monkeys received unilateral carotid injections of MPTP. They were divided in an "asymptomatic" (A) group (N = 3) and a "symptomatic" (S) group of four monkeys with mild to profound impairments. 3 - $5 \mathrm{mCi}$ of (C-11) raclopride (specific activity $134 \pm 32 \mathrm{Ci} / \mathrm{mmoles})$ were injected. The ratios of the basal ganglia (caudate and putamen) to a non-specific area (cerebellum) 30 - 40 min post injection were compared using unpaired t-tests.

Due to the large inter-animal variability and the small number of subjects, there was no significant change in striatal raclopride binding between the control and the $A$ and $S$ groups. There were significant differences between the right-left differences in the lesioned monkeys compared to normals suggesting 1) a decrease binding in the MPTP-injected striatum in the A group and 2) an increase binding in the MPTP-injected striatum in the $S$ group.

The increase in raclopride binding in the symptomatic animals is in agreement with previously reported data in parkinsonian patients. The decrease in raclopride binding in the asymptomatic animals may be attributed either to compensatory mechanisms or to intrastriatal cell loss in addition to the nigro-striatal pathway.

\section{P.76}

Routes of Administration and Effect of Carbidopa Pretreatment on 6-[ ${ }^{18}$ F] Fluoro-L-Dopa/PET Scans in Nonhuman Primates

\section{G.L.Y. Chan, D.J. Doudet, T. Dobko, K. Hewitt, P. Schofield,} B.D. PATE and T. RUTH (Vancouver, British Columbia)

In 6-[ $\left.{ }^{18} \mathrm{~F}\right]$ fluoro-L-dopa (FD)/positron emission tomography (PET) studies, carbidopa (CD) pretreatment increases the FD availability to the brain and enhances the intensity of striatal PET images. Different research teams have used various CD doses and routes of administration in non-human primate studies. The purpose of this study was to examine the plasma profiles of $C D$ and the effect of the route of administration of $C D$ on a FD/PET scan. Cynomolgus monkeys were given $C D$ either orally $(5 \mathrm{mg} / \mathrm{kg})$, intraperitoneally (IP) $(2.5$ and $5 \mathrm{mg} / \mathrm{kg})$ or intravenously (IV) $(5 \mathrm{mg} / \mathrm{kg}) 60$ - $90 \mathrm{~min}$ prior to the FD injection. Carbidopa-treated monkeys were compared to monkeys without CD-pretreatment. No CD was detected in the plasma samples when it was given orally, possibly due to poor absorption in the gastrointestinal tract. In addition, the striatal and cortical activities were not statistically different from those of the untreated monkeys, indicating that little or no peripheral decarboxylation inhibition of FD by CD had taken place. When CD was given IP at a dose of 2.5 and $5 \mathrm{mg} / \mathrm{kg}$ and IV at $5 \mathrm{mg} / \mathrm{kg}$, plasma CD levels at the time of FD injection were $0.95 \pm 0.26,2.22 \pm 0.23$ and $2.79 \pm 0.26 \mu \mathrm{g} / \mathrm{ml}$, respectively. Due to peripheral decarboxylation inhibition of FD by $\mathrm{CD}$, more FD was available for transport into the brain. As a result, both the striatal and cortical activities were significantly higher than those of the controls. Carbidopa pretreatment had no effect on either the striatal-tocortical activity ratio or the striatum uptake value.

\section{P-77}

Some Complex Aspects of Language and Reasoning Are Impaired in Non-demented Parkinsonian Patients

Emmanuelle Pourcher, Henri Cohen, Paul J. Bédard and JEAN-PIERre Bouchard (Quebec, Quebec)

Introduction: Only recently, cognitive impairment has been recognized as a feature of Parkinson's disease (PD), a neurodegenerative disorder resulting primarily in a dopaminergic deafferentation of striatal structures. Language functions seem well preserved using standard aphasia batteries. However observations of deficits in tests tagged as frontal function tests raise the question as to whether more complex linguistic processes may be impaired. Methods: We compared the performance of 38 non-demented, highly-functioning PD and 19 age and education matched controls on the following tests: verbal fluency, California Verbal Learning Test, Similarities, a lexical test, an invited inference test and a test of logical reasoning on verbal material. Results: Multivariate analysis of variance confirmed normal performances in tasks of letter and category fluency, and on a lexical test; learning capacity despite observed memory deficit appears also preserved. Conversely specific deficits in similarities and logical reasoning were observed suggesting that tasks requiring new algorithmic strategies, in contrast to tasks requiring pragmatic or heuristic approaches are likely to be difficult to achieve. Impairments in conceptual fluency and reasoning abilities with new strategies may constitute one of the multiple aspects of the impairment of the so-called executive functions in Parkinson's disease.

\section{EPILEPSY $(P-78$ to $P$-90)}

\section{P-78}

Insulin Dependent Diabetes Mellitus (IDDM) in Three Children With Epilepsy

\section{R.I. MUNN (Toronto, Ontario)}

Three boys with normal development (two Italian, one Greek) developed seizure disorders at a mean age of 7.2 years. Two had benign rolandic epilepsy (hemifacial seizures with rolandic spikes) and the third had early morning generalized tonic-clonic seizures with focal EEG abnormalities which varied in location over time. All of the children had complete seizure control with carbamazepine and discontinued the medication with normal EEG's at an average age of 11.5 years.

IDDM developed (average age of onset 11.3 years) in two of the boys while on carbamazepine and in the third shortly after 
discontinuing the medication. There was a family history of both epilepsy and Type-II diabetes in two of the children. There was no family history of Type-I diabetes.

Genetic factors are important in the etiology of both childhood diabetes and epilepsy. The three children described questions whether a genetic link might exist between IDDM and epilepsy in some children.

\section{P-79}

\section{Cryptogenic Temporal Lobe Epilepsy (TLE) in Children}

Mecheri Sundaram and V. Vedanarayanan (Jackson, Mississippi)

Information on childhood TLE is limited. We present clinical data on twelve non-retarded children with EEG proven TLE attending our neurology clinics (age: 2 - 16). All of them had seizure onset before the age of 10 with a minimal follow-up of two years. MRI showed medial temporal sclerosis in five, neocortical gliosis in one, venus angioma in one, no abnormality in five. No birth insult could be identified in any patient. Family history for seizures was positive in one. Complicated febrile seizures occurred in one. Total number of seizures was less than ten in eleven children. Excellent control was achieved in all of them with monotherapy.

Conclusion: 1). Medial temporal sclerosis can be seen in children with cryptogenic TLE without obvious birth insult or intractable epilepsy; 2). Febrile seizures in this clinical series of TLE is rare; 3 ). Prognosis for seizure control appears excellent in spite of non-progressive structural pathology.

\section{$\mathbf{P - 8 0}$}

\section{The Epidemiology of Clinical Neonatal Seizures. A Five-year} Cohort in Newfoundland

G.M. Ronen and S. PEnNEY (St. John's, Newfoundland)

Study Question: What is the incidence and distribution of the clinical features and etiologies in clinical neonatal seizures (NS)? Objective: use current concepts to delineate the spectrum of clinical NS within a population setting. Methods: 1. Sessions on NS symptomatology to neonatal and maternity nurses across the island. 2. Detailed questionnaires prospectively collected and verified, on all consecutive NS from 1.1.1990 - 31.12.94 at the single tertiary neonatal unit in Newfoundland, where traditionally all neonates with seizures are admitted. Results: Entered 94 neonates. Incidence of $2.5 / 1000$ live births was calculated for 87 neonates with either focal seizures or not being triggered by stimuli or abolished by restraint. $78 \%>35$ weeks gestation, $64 \%$ with seizure onset in the first 48 hours, $79 \%$ had seizures lasting $<5 \mathrm{~min}$ and $6 \%$ status $(11 \%<35 \mathrm{w}) .44 \%$ had $<5$ seizures, $40 \%$ had $>10$ seizures per patient. Symptomatology showed mixed seizure pattern in $68 \%$, clonic movements in $76 \%$, unilateral clonic $44 \%$, bilateral clonic $47 \%$, tonic eye deviation $18 \%$, unilateral tonic $6 \%$, generalized tonic $22 \%$, subtle $48 \%$, myoclonic $10 \%$, apnea $37 \%$ and cyanosis 46\%. Etiology: $48 \%$ asphyxia, $17 \%$ metabolic derangement, $16 \%$ infections, $14 \%$ extra parenchymal hemorrhage, $9 \%$ congenital malformations, $8 \%$ genetic predisposition, and $6 \%$ parenchymal infarcts/hemorrhages. Neonatal death occurred in $8 \%, 17 \%$ in $<35$ weeks and $12 \%$ in the asphyxiated neonates.

\section{P-81}

The Absence of Significant Serum Calcium Abnormalities in New Onset Non-febrile Seizures in Children

Parbeen Pathak and D.L. Keene (Ottawa, Ontario)

Background: Children presenting to pediatric emergency rooms, with seizures, routinely have serum chemistry testing. Previous studies have shown that such testing is not useful. Objectives: 1) To determine what percentage of patients, who presented with new onset non-febrile seizures, had testing of serum calcium in a pediatric ER. 2) To report the frequency of serum calcium abnormalities in this group. Methods: 1) Retrospective study reviewed the charts of patients who presented over a 6-month period (April 1 - Sept. 30, 1994) with new onset seizures. 2) Only non-febrile seizures were studied. 3) Age limitation: Older than 28 days and younger than 18 years of age. Results: Mean age: 7.7 years. 60 patients identified. 41/60 patients had measurement of serum calcium (68.3\%). $7 / 41$ cases had serum calcium levels above the laboratory established upper limit of normal. 34 cases were normocalcemic. No specific therapy required for the serum calcium abnormalities. Conclusion: Routine measurement of serum calcium in pediatric patients with first time non-febrile seizures, is not useful.

Characteristics of Epilepsy During Adolescence: Study of a Clinic Population

A. Ogunyemi, S. Penney, V. Gadag, D. Buckley and W. BAvington (St. John's, Newfoundland)

Few studies have described the characteristics of adolescent patients with epilepsy. We examined several characteristics of all the patients who attend the Adolescent Seizure Clinic at the Charles Janeway Child Health Centre, St. John's, Newfoundland.

There were 165 patients aged 8 to 19 years. 69 were female and 96 male. 58 patients had generalized seizures, 94 had partial seizures while 13 had both partial and generalized seizures. $52 \%$ were seizure-free for one year or longer, $30 \%$ were having two or more seizures per month and $18 \%$ had one seizure or less per month.

The male and female patients did not differ with respect to seizure type or seizure frequency. Seizures began at younger ages in those with partial seizures compared with the ones having generalized seizures. Also, patients with partial seizures were receiving significantly more anti-seizure medications when compared with those who had generalized seizures.

Our study supports the viewpoint that adolescent patients with partial seizures fare worse than those with generalized seizures. 
Epileptiform Activity in Children With Landau-Kleffner Syndrome: Analysis of Digitized EEG Mapping and VideoEEG Telemetry

H. Otsubo, P.A. Hwang, A. Hunjan, M. Cortez, J. Kobayashi and W. RoBERTS (Toronto, Ontario)

Landau-Kleffner syndrome (LKS) consists of acquired aphasia with seizures and generalized spike-waves on EEG. Whether speech impairment is due to the epileptiform activity or an underlying brain abnormality is still uncertain. We analyzed epileptiform discharges of children with LKS to define location and extent of the epileptic activities by digitized EEG mapping. EEGs were performed with regular 10 - 20 scalp electrodes using "Monitor" and "Rhythm" (Stellate) for digitized recording. Split-screen video telemetry was recorded by "System 4000 " (BMSI). Eight patients aged two to nine years with LKS were evaluated. Neurological examinations and neuroimaging studies were normal in all. Behavioural problems were noticed in seven. Four patients had history of seizures. 19 EEGs were recorded with computerized digitized data, including four prolonged video-telemetry. Continuous spike and slow waves during slow-wave sleep were seen in two patients. Multifocal epileptiform activities over frontal, temporal, and parietal regions were found in five, including horizontal dipole phenomena over rolandic or sylvian fissure were demonstrated on EEG mapping. Based on the spatial and temporal compterized analysis of EEG in these patients, epileptic phenomena, either tangential dipole epileptiform discharges, are contributory to the underlying abnormalities of speech areas.

\section{P-84}

\section{Clobazam and Metabolite Serum Levels Show Little} Relationship to Dose

\section{Canadian Clobazam Study Group (Halifax, Nova Scotia)}

A multicentre Canadian randomized trial of monotherapy clobazam for children with epilepsy allowed examination of relationships between dose $(\mathrm{mg} / \mathrm{kg} / \mathrm{day})$ and serum level of clobazam and its biologically active metabolite, n-desmethylclobazam (NDMC). Serum was obtained predose in steady state and analyzed by high pressure liquid chromatography in a central laboratory (Dr. A. Fraser, Halifax). 190 levels were available from 97 children. Clobazam dose ranged 20 fold $(0.1-2.0$ $\mathrm{mg} / \mathrm{kg} /$ day, mean $0.64 \pm 0.3$ ). Serum levels of clobazam ranged widely $(0.3-5.6 \mathrm{~mm} / \mathrm{l}$, mean $1.4 \pm 0.8)$ as did NDMC $(0.4-50$ $\mathrm{mm} / \mathrm{l}$, mean $4.3 \pm 6.3$ ). A plot of serum clobazam level versus dose showed wide scatter with modest statistical correlation $(r=$ 0.37). NDMC levels were not related to clobazam dose or level. Age and sex did not influence levels (multiple regression). 90 patients had $\geq 2$ levels. If the dose was unchanged, increased or decreased by $\geq 10 \%$, levels did not consistently stay the same or change in the expected direction. Therefore, serum clobazam and NMDC levels have little relationship to dose. Correlation between these variable levels and efficacy or toxicity is needed to establish clinical utility.

\section{Therapy-resistant Cryptogenic Partial Epilepsy Beginning in Childhood and Adolescence}

\section{W.T. Blume, D. LeE, D. Munoz and S. Wiebe (London, Ontario)}

All patients with therapy-resistant partial seizures without significant pathological lesions and/or without demonstrable MRI pathology who underwent in-patient investigation in our Epilepsy Unit will be reviewed. With current radiological-pathological techniques, this group falls between the benign partial epilepsies of childhood and lesion-related partial epilepsies.

\section{P-86}

Seasonal Onset of Infantile Spasms and the Possible Role of Zeitgebers

\section{M.A. CoRTez and P.A. Hwang (Toronto, Ontario)}

A chronobiological approach of Infantile Spasms (IS) is presented. IS is a severe multifactorial epileptic syndrome associated with hypsarrhythmia, decreased REM sleep and poor developmental outcome. Zeitgeber is a time cue. Sunlight is known to set network of biological rhythms according to an appropriate time scale, such as 24 hours. Objective: To determine whether the occurrence of IS presents with seasonal variation. Design: Retrospective review of randomly selected cases. Methods: Health records of 32 infants whose IS occurred during the first year, presenting between 1984 - 1993, were reviewed. Six cases were excluded, and 26 met EEG criteria of hypsarrhythmia. The months of IS onset were plotted to produce a frequency histogram. The number of cases in twomonth intervals was examined for seasonal variation and clustering. Results: Of $14 \mathrm{~F}$ and $12 \mathrm{M}, 21$ were symptomatic and 5 cryptogenic. Onset of IS ranged from two to nine months (mean $=5.4$ ). A seasonal peak occurred in Nov. - Dec. with nine cases (34.6\%) compared with three cases (11\%) in May - June, (mean $=4.3$ bimonthly). Poorly organized sleep EEG features was reported in all cases. Conclusions: The occurrence of IS appears greatest in late fall (Nov. - Dec.) for unknown reasons. The possible role of zeitgebers in IS is suggested.

\section{P.87}

\section{The Prognosis for Seizures in Primary Autism is Relatively Good}

N.J. Lowry, Karen Streilein and Esther Cherland (Saskatoon, Saskatchewan)

Forty-five children with autism were seen and followed over a 10 year period. 15 of $45(33 \%)$ had seizures. 10 of 15 have primary (Kanner's) autism and five have autism associated with other neurological diagnoses - one has tuberous sclerosis, one has Soto's syndrome, one has severe sensorineural hearing loss and two have the Lennox-Gastaut syndrome. The commonest seizure types seen were generalized motor seizures (9), complex partial seizures (6), and atypical absence's (6). 60\% had more than one type of seizure. 10 of 15 are seizure free at follow-up (four off anticonvulsants, six still taking anticonvulsants). 
Persistent seizures were seen in the two cases of LennoxGastaut, one tuberous sclerosis and two cases of primary autism. 9 of $11(80 \%)$ of primary autism patients are seizure free (on or off medication) at follow-up.

\section{P.88}

The Positive Effects of Piracetam on a Patient With Intention Myoclonus and Turncal Ataxia Following Hyponic Encephalopathy

\section{B.M. Chaudhuri (Calgary, Alberta)}

This case will demonstrate the positive effects of Piracetam over a relatively short time period on a 22 -year-old patient with action myoclonus and truncal ataxia. Piracetam has been used in Europe for a long time for cognitive protection after hypoxic events and after the onset of Parkinson's disease.

The patient in this case improved dramatically over a six week period. He moved from being wheel chair bound to using a walker and finally to using a cane without assistance. This drug was tried after the traditional therapies for intention myoclonus were implemented including with up to $10 \mathrm{mg}$ of clonazepam and $2000 \mathrm{mg}$ of Epival daily for four months before the Piracetam was initiated. The patient is now taking $12 \mathrm{~g}$ of Piracetam per day.

This poster will explore the nature of Neurotropic drugs, of which Piracetam is one, and will also explore the physiology and pharmacology of this drug. Piracetam has virtually no side effects and the rapid improvement of this patient has remarkable implications for its future applications.

\section{P-89}

\section{Withdrawn}

\section{P-90}

\section{Weight Gain on Valproic Acid}

Nathalie Leung, Céline Corman, Denis Boisvert and Alan GubERMAN (Ottawa, Ontario)

Valproic acid (VPA) has become one of the first line antiepileptic drugs for a variety of seizure types. Weight gain has been recognized as a common adverse effect and leads to discontinuation relatively frequently. Only few publications have attempted to address the incidence and correlates of weight gain on VPA.

We have analyzed the records retrospectively and interviewed 70 adult patients attending an epilepsy clinic on VPA mono- or polytherapy followed over a median of 27 months (range 3 - 189 months) as well as 20 patients on carbamazepine (CBZ) monotherapy. Patients were divided into non-weight gainers ( $<5 \%$ baseline body weight), mild-moderate weight gainers ( 5 - 10\% body weight) and marked weight gainers (> $10 \%$ body weight). The following variables were statistically analyzed to determine their relationship to weight gain: sex, concurrent therapy, personal or family history of obesity, drug dose and serum level, changes in eating or exercise habits, seizure control and other adverse drug effects.

Preliminary analysis showed that $71 \%$ of the VPA group were weight gainers vs. $43 \%$ in $\mathrm{CBZ}$ group and that only drug dose and female sex correlated with weight gain. Weight gain was often sustained and frequently socially significant to the patients. Possible strategies to reduce this adverse effect include dietary and exercise counselling, dose reduction and careful patient selection.

\section{NEUROSURGERY - TUMOURS $(P-91$ to $P$ - 102$)$}

\section{P-91}

C6 Astrocytoma Cells Release an Extracellular Proteasomelike Structrue With Collagenase I, IV and General Protease Activity

R. Del Maestro, Indrasen Vaithilingam and Warren MCDONALD (London, Ontario)

The serum-free media conditioned by $\mathrm{C} 6$ astrocytoma cells contains a novel extracellular proteasome-like structure (EP) which is composed of three subunits, two isoelectic variants at $70-\mathrm{kDa}$ and one at $65-\mathrm{kDa}$ and a molecular mass of $1000-\mathrm{kDa}$. Electron microscopic studies demonstrate that EP has a circular structure of $11 \mathrm{~nm}$ diameter which, on end-on view, is an elongated cylinder. Collagen type I and type IV, $\alpha$-casein, $\beta$-insulin albumin and a number of synthetic peptides are substrates for EP. The EP accounts for about $50 \%$ of the collagenase IV activity released by $\mathrm{C} 6$ cells, but this is variably expressed during monolayer culture. The type IV collagenase activity of the proteasome was sensitive to serine inhibitors while the general protease activity was sensitive to metalloprotease inhibitors. The location of EP in the extracellular space is not the result of cell lysis since the ratio of extracellular to intracellular lactate dehydrogenase was not consistent with this. The intracellular origin of EP was confirmed by utilizing ${ }^{35} \mathrm{~S}$ methionine. The novel features of the proteasome include (i) its release from tumour cells, (ii) its high subunit molecular weight, (iii) its ability to degrade 
collagen type I and type IV. Summary: The specificity of EP along with its multicatalytic capacity suggests that EP may play a significant role in the initiation of angiogenesis. The development of strategies for tumour-associated angiogenesis inhibition must take the presence fo extracellular proteasome into consideration.

\section{P-92}

\section{Concurrent Brain Tumours}

\section{VASSILYADI and R.P. PokruPa (Montreal, Quebec)}

Concurrent brain tumours are uncommon, but do occur in patients not irradiated. A 64-year-old female with ammenorrhea since the age of 36 , and morbid obesity, presented with dizziness, weakness, and two sudden episodes of vomiting. On examination she had galactorrhea and decreased axillary hair. Visual acuity was 20/25 bilaterally, however, mild optic atrophy was present. Hypothyrodism, hypoadrenalism, and a serum prolactin of 1460 were discovered. Two lesions were identified on brain MRI, with variations in their uptake of contrast.

The patient was started on cortisone, levothyroxine, and bromocriptine. Seven weeks later the serum prolactin decreased to 8.7. She underwent a right pterional craniotomy and gross total resection of the tuberculum sellae meningioma. The diaphragma sella was left intact.

The world literature reports only ten cases of concurrent suprasellar meningiomas and pituitary adenomas, the latter usually being either nonsecreting or growth hormone secreting tumours. Only once was a concurrent tuberculum sellae meningioma and a sellar prolactinoma previously reported. The possible oncogenesis of meningiomas and pituitary adenomas, and their coexistence, is discussed.

\section{P-93}

\section{Epidemiology of Childhood Brain Tumours}

Darlene M. Miltenburg, Deon F. Louw and Garnette R. SUTHERLAND (Winnipeg, Manitoba; Calgary, Alberta)

Brain tumours comprise more than $20 \%$ of all childhood malignancies, and constitute the greatest number of solid pediatric cancers. Incidence rates reported have been varied, reflecting the impact of modern imaging techniques, the application of diverse investigative methodologies, and the accessibility of the community to health care.

Material was collated from the Manitoba Cancer Foundation Tumour Registry and from the personal records of Winnipeg pediatric neurologists. Patient data were also obtained from hospital charts and operating room log books. Histological sections were examined and classified according to the American Cancer Society by a single neuropathologist. The chisquare test was used for statistical evaluation.

During the seven year study period, the diagnosis of brain tumour was made in 88 pediatric patients. The overall average annual incidence rate for both sexes was $4.0 / 100,000$ childyears, higher than that previously reported. The male and female average incidence rates were 4.2 and $3.7 / 100,000$ child-years, respectively. Tumour type and location were relatively unremarkable, an expected peak of medulloblastoma occurring in young males. The yearly incidence of tumour occurrence was fairly stable, and the geographic distribution of cases within Manitoba, homogeneous.

\section{P-94}

The Production of Collagenase IV in C6 Astrocytoma Monolayer Culture Depends on the Cell Density

M. TAMAKl, Warren MCDonald and Rolando F. Del MAESTRO (London, Ontario)

Background: Angiogenesis is a process by which new blood vessels are formed and is essential for the growth of solid tumours. Since collagen type IV (Col IV) is a major component of the basement membrane, collagenase IV is crucial in angiogenesis. We investigated the following points: (1) What the influence of cell density and time in culture have on the release of collagenase IV? (2) Which enzymes are modulated during cell growth? Methods: C6 astrocytoma cells were seeded at three different densities $\left(5000,10^{4}, 10^{5}\right)$ and conditioned media was collected daily over five days. Collagenase activity in the conditioned media was assayed by measuring the degradation of ${ }^{3} \mathrm{H}$-collagen IV and by Western blot analysis. Immunocytochemistry was also performed to investigate the expression of intracellular gelatinase A. Results: (1) Collagenase IV activity per cell decreases with increasing cell density and time of cell growth in culture; (2) Western blot analysis of gelatinase A showed similar results; (3) Immunocytochemistry indicated that gelatinase A decreased in the same manner intracellularly. Conclusion: These findings indicate the existence of a regulatory mechanisms for the production and release of collagenase IV. Individual or small groups of cells release substantially more enzyme per cell which may be essential to cell invasiveness and the control of angiogenesis.

\section{P-95}

\section{Lipomas of the Scalp: Case Review and Report}

\section{Kym L. Chandler (Memphis, Tennessee)}

Pure lipomas of the scalp are rare. At presentation, these lesions are small and clinically are similar to lipomas found on the rest of the body. When they do involve the skull, they tend to arise from lipocytes within the diploic marrow. Therefore, bony involvement is evident early in the clinical course. Common scalp lesions such as dermoids and epidermoids routinely involve the skull, progressively thinning it secondary to chronic external pressure rather than intramedullary involvement. This produces characteristic radiographic and clinical findings. To date, however, there has been no report of a benign lipoma of the scalp eroding the skull instead of originating within the diploe. We report a case of a huge lipoma of the scalp that slowly enlarged over 18 years, eroding the skull in a manner similar to that observed with dermoids and epidermoids. Methylmethacrylate cranioplasty was necessary to obliterate the skull defect. On the contralateral side of the scalp, another much smaller lesion present for two years was observed and removed. In this instance, there was no bony involvement. A review of the literature demonstrates that this case is unusual not only for the presence of bony erosion and tumour size but also for the bilaterality of the lesions. 
P-96

\section{Neuroblastoma of Pituitary Gland with Hyperprolactinemia}

B.G. Benoit, B. Lach, P.R. Garner, P. Rippstein and B. STAINES (Ottawa, Ontario)

This is the first case report of an intra/supra sellar neuroblastoma involving the adenohypophysis. Associated prolactin secretion caused an amenorrhea-galactorrhea syndrome.

A 40-year-old woman with irregular menses since menarche presented with a 12-year history of infertility and galactorrhea with hyperprolactinemia. She was intolerant of Bromocriptine and Pergolide. Repeat CT/MR scans show an enlarging macroadenoma. Six months prior to surgery, she complained of "blurred vision" and was found to have a bi-temporal defect. At craniotomy, a large fibrous tumour was removed and visual function returned to normal. A six month routine follow up MR showed residual/recurrent tumour, which led to a second resection one year later, followed by radiotherapy. The prolactinemia subsided.

Light microscopy, immuno-electron microscopy, and immuno-histochemical studies showed features typical of peripheral neuroblastoma, with cells positive for oxytocin, and nests showing prolactin granules in the epithelial cells and neuritic processes. Several mechanisms could explain the clinical hyperprolactinemia.

\section{P.97}

Intracranial Suprasellar Angiolipoma: Ultrastructural and Immunohistochemical Features

\section{H.J. LesIUK, B. LACH and B.G. BeNOIT (Ottawa, Ontario)}

The authors present ultrastructural and immunohistochemical characteristics of an intracranial suprasellar tumour displaying features of cavernous angioma with islets of adipose tissue. Electron microscopy revealed thin-walled vessels separated by a loose collagenous stroma containing nests of mature adipocytes as well as fibroblasts, myofibroblasts, mast cells, and a few macrophages. Intracytoplasmic lipid droplets were also identified in scattered pericytes and smooth muscle cells of vascular walls and in the transitional cells resembling smooth muscle cells and adipocytes. Many adipose tissue cells were positive for S-100 protein with polyclonal antibodies. Other lipidized tumour cells were immunoreactive for some or all of the following: smooth muscle-specific actin, factor XIIIa, vimentin, and, occasionally, for desmin. Ultrastructure and immunohistochemistry indicate that in addition to typical adipocytes, lipidized cells of another nature contribute to the characteristic appearance of the adipose tissue component of angiolipoma.

\section{P-98}

\section{Adult Brainstem Gliomas: Clinical, Radiological and Pathological Features}

Michael Tymianski, M. Cusimano and J. Bilbao (Toronto, Ontario)

Gliomas of the brain stem comprise $2 \%$ of adult neuroepithelial tumours. Between 1981 and 1994, six patients with such tumours were managed at our institution (Table 1). All underwent imaging with CT and/or MRI, and open biopsy of their lesion. All patients initially presented with signs of local brain stem dysfunction, including cranial nerve palsies, ataxia, and long tract signs. None had hydrocephalus, none suffered from raised intracranial pressure. The rate of progression of clinical features related solely to the tumour histology, with high grade lesions having a rapid, relentless course culminating in death within a short period. One patient, initially diagnosed with a low grade lesion, succumbed when the lesion transformed into a glioblastoma. In contrast with their supratentorial counterparts, high grade lesions enhanced variably with gadolinium on the MRI scan, whereas high enhancement was always observed with low grade lesions. Given the variable appearance of high grade lesions on MRI, and their poor prognosis, a tissue diagnosis should be obtained for diagnostic prognostic reasons prior to prescribing further therapy for all adults with brainstem tumours.

\begin{tabular}{|c|c|c|c|c|}
\hline Age & Histology & Site & Sx (months) & $\mathrm{F} / \mathrm{U}(\mathrm{mos})$ \\
\hline 17 & Astrocytoma & Medulla & 36 & 20 (alive) \\
\hline 51 & Pilocytic astro & Medulla & 21 & (alive) \\
\hline 29 & GBM & Medulla & 1 & (dead) \\
\hline 36 & Malignant astro & Pons & 7 & (dead) \\
\hline 24 & Astro $->$ GBM & Pons & 24 & 17 (dead) \\
\hline 53 & GBM & Pons & 1 & 0.5 (dead) \\
\hline
\end{tabular}

\section{P-99}

An Unusual Lymphoid Mass of the Intracranial Meninges Presenting as Meningioma

E.V. Colapinto, N. Razack and L. Spollen (Columbia, Missouri; Manhasset, New York)

Rare inflammatory masses attached to the dura may simulate the gross and radiological appearance of meningioma. A 59-yearold right-handed female presented with a three month history of forgetfulness and speech difficulties. Physical examination revealed dysphasia, decreased short-term memory, and right-sided weakness. Computed tomographic scan of the brain showed an enhancing mass over the left frontal cenvexity with surrounding cerebral edema. The patient underwent left frontal craniotomy and the extra-axial dural-based mass was resected. The patient had resolution of her neurological deficits and has remained well 13 months after surgery. Pathological examination of the surgical specimen showed a partially nodular and partially diffuse lymphoid infiltrate. Many large and small lymphoid cells were atypical with pale cytoplasm and irregular nuclei. Immunohistochemical stains showed mixed $B$ and $T$ cell populations, and flow cytometry demonstrated no monoclonality. Gene rearrangement studies were negative. This unusual intracranial lesion differs from previously described inflammatory meningeal masses due to the atypicality evident in the lymphoid infiltrate, the lack of plasma cells or sclerosis, and the absence of any meningothelial cells to suggest an underlying meningioma. The etiology and classification of this heterogeneous group of lesions remains uncertain, but most can be surgically resected and adjuvant therapy is probably not necessary. 
P-100

The Effect of Blood on the Transmission of $630 \mathrm{~nm}$ Light Through a Diffusion Medium Used in the Photodynamic Therapy of Brain Tumours

\section{P.J. MULLER and B.C. WiLSON (Toronto, Ontario)}

In the photodynamic therapy of brain tumours a balloon irradiator filled with a light dispersion medium 1:1000 dilution of sterile Nutralipid or Intralipid [Pharmacia (Canada) Inc., Dorval, Quebec] coupled to an Argon pumped dye laser (Model 880 or Model Aurora M laser, Cooper Medical, Mountain View, California) is used for cavitary photoillumination after tumour extirpation at craniotomy. An alternate method of cavitary photoillumination has been to fill the tumour resection cavity with the light dispersion medium and place an optical fiber into the centre of the resection cavity. The diffusion medium is often contaminated by blood during the photoillumination.

We used a phantom tumour cavity to measure the back scatter of $630 \mathrm{~nm}$ light over a $10 \mathrm{~mm}$ distance from a $2 \mathrm{~cm}$ diffusion tip fiber placed in medium contaminated with various concentrations of blood.

There was an initial steep fall in the back scatter at $\mathrm{Hb}$ concentration of 0 to $2 \mathrm{~g} / \mathrm{l}$ and then a smooth slope suggesting a bimodal light attenuation. A $10 \%$ reduction occurred at $0.4 \mathrm{~g} / \mathrm{l}$ and a $50 \%$ reduction at $6.0 \mathrm{~g} / \mathrm{l}$. Continuous replacement of the medium in order to keep the $\mathrm{Hb}$ level below $0.5 \mathrm{mg} / \mathrm{l}$ will prevent the attenuation of the light flux.

\section{P-101}

\section{Tuberculum Sellae Meningiomas: A Short Series}

\section{S. Konasiewicz, P.J. Muller, W. Tucker, R.J. Moulton and J.} BilbaO (Toronto, Ontario)

Tuberculum sellae meningiomas (TSM) are tumours arising from the post posterior surface of the planum sphenoidale at the tuberculum of the sella. Their clinical presentation consists of a homonymous hemianopsia and/or visual failure with optic atrophy. In our data bank we found five TSM is a series of 401 patients (1.2\% of miningiomas). There were three females and two males. All presented with visual failure, three had optic atrophy and only one an enlarged sella. In one patient the tumour was huge (long diameter $=6 \mathrm{~cm}$ ); he underwent an orbital osteotomy approach with bilateral frontal craniotomy. One patient had a transsphenoidal approach (preoperative diagnosis was pituitary adenoma) and three had standard pterional approaches.

In a similar interval 112 pituitary macro adenomas were identified. Of those patients with tumours between 1 and 2.4 $\mathrm{cm}$ diameter, $25 \%$ presented with visual findings; of those with tumours $>2.5 \mathrm{~cm}$ diameter, $75 \%$ presented with visual findings.

The radiographic and MRI features can distinguish TSM from pituitary adenomas. TSM are homogeneously enhancing lesions with a dural tail and a normal sized sella.
P-102

\section{Update on Photodynamic Therapy (PDT) for Recurrent Supratentorial Gliomas}

\section{P.J. MULLER and B.C. WILSON (Toronto, Ontario)}

PDT was used in 56 patients with recurrent supratentorial gliomas who had failed radiation therapy and who were candidates for palliative reoperation. Porphyrin photosensitizer was administered i.v. 12 - 36 hours prior to photoillumination. All patients had the recurrent tumour subtotally resected or cyst drained followed by intraoperative cavitary photoillumination. In 15 cases interstitial photoillumination using fibers with 2 $\mathrm{cm}$ diffusing tips supplemented the cavitary illumination. The total light energy delivered ranged from 440 to $4500 \mathrm{~J}$ (median $=1800 \mathrm{~J})$. The energy density ranged from 8 to $110 \mathrm{~J} / \mathrm{cm}^{2}$ $\left(\right.$ median $\left.=38 \mathrm{~J} / \mathrm{cm}^{2}\right)$.

The post-PDT median survival of patients with recurrent GBM was 31 weeks with a one and two year actuarial survival of $18 \%$ and $0 \%$, respectively. The GBM median survival GBM from first diagnosis was 118 weeks with a one and two year rates of $82 \%$ and $57 \%$, respectively. For recurrent MA the post-PDT median survival was 44 weeks with one and two year rate of $43 \%$ and $36 \%$, respectively; and, first diagnosis the median survival was 147 weeks with a one and two year rate of $86 \%$ and $71 \%$, respectively. Patients with MA who received greater than the median light dose had a longer survival than those who received less than the median light dose. With increasing light doses, better light delivery systems and more effective photosensitizers further improvement in survival can be expected.

\section{NEURO-ONCOLOGY (P-103 to P-115)}

\section{P-103}

Intravascular Lymphoma: a Rare Cause of Multifocal Neurologic Disease

\author{
M.J. Wong, J. MAH and J. Holden (Vancouver, British \\ Columbia)
}

Intravascular lymphoma (neoplastic angioendotheliomatosis or malignant angioendotheliosis) is a rare malignancy characterized by neoplastic cells within the lumen of capillaries, small veins and arteries. Initially it was felt to be an endothelial neoplasm because of a positive staining for factor 8 antigen, a specific antigen marker of endothelial cells. However, based on histochemical staining, these cells are felt to arise from a monoclonal lymphoid population of either B or, less frequently, $\mathrm{T}$ cell lineage. Therefore, the modern terminology for this neoplasm is angiotropic lymphoma or intravascular lymphoma.

Although this disease can present with systemic manifestations, particularly skin lesions, $60 \%$ will have CNS complications. There are only 40 reported cases presenting with primarily CNS disease.

Because of the rarity of this disease, the diagnosis is usually made post mortem. This diagnosis should be considered in patients presenting with bizarre and multifocal neurological signs. Newer treatment modalities, such as chemotherapy and plasmaphoresis, may temporarily halt the progression of disease. In the absence of systemic disease, meningeal or brain or peripheral nerve biopsy should be considered. We would like to present the clinical and pathological features of such a case. 


\section{P-104}

Paraneoplastic Limbic Encephalitis in Association With Non-Hodgkin's Lymphoma

Hannah R. Briemberg, J. Turnbull and J.A. Maguire (Toronto, Hamilton, Ontario)

An 81 -year-old man presented with rapidly progressive dementia, with confusion, personality changes, incontinence and falls progressing to death six months later. Spinal fluid had increased protein and moderate lymphocytosis but no malignant cells. MRI showed frontal atrophy with multiple areas of increased $\mathrm{T} 2$ signal intensity in the left thalamus, basal ganglia and white matter of the frontal lobes extending across the corpus callosum. The patient developed thrombocytopenia and bone marrow biopsy showed well-differentiated low grade lymphoma. Autopsy confirmed systemic B-cell lymphoma with metastases to the corpus callosum and periventricular regions. There were also extensive parenchymal and perivascular $\mathrm{T}$ cell infiltrates, consistent with a paraneoplastic syndrome, in the corpus callosum, hippocampi, cingulate gyri, basal ganglia, midbrain, pons, and cerebellar dentate nuclei. The basal ganglia and cingulate gyri had extensive gliosis, and spongiform changes were noted in the centrum semi-ovale and corpus callosum extending to both cingulate gyri. This is the first pathologically confirmed report of paraneoplastic limbic encephalitis associated with lymphoma. Though rare, lymphoma causing limbic encephalitis must be considered in atypical dementia.

\section{P-105}

A Prospective Randomized Study of Prophylactic Anticonvulsants (AC) in Patients with Primary Brain Tumours (PBT) or Metastatic Brain Tumours (MBT) and Without Prior Seizures (Sz): A Preliminary Analysis of 67 Patients

P.A. Forsyth, S. Weaver, D. Fulton, P. Brasher, N. Hagen, G. Sutherland and L. Deangelis (Calgary, Alberta; Albany, New York, New York; Edmonton, Alberta)

Objectives: 1) To determine if AC given prophylactically to BT patients reduces $S z$ frequency, 2) To describe the type of Szs, and 3) The toxicity of AC in BT patients. Background: $\mathrm{Sz}$ occur in $20-30 \%$ of patients with a PBT or MBT. AC are commonly used prophylactically despite the absence of proof for efficacy in BTs and potential side effects. Methods: We conducted a prospective study of prophylactic $A C$ in new BT pts. Pts were randomized within one month to receive AC (AC Grp.) or no AC (No AC Grp.); pts with prior Sz were excluded. All had a KPS of $>50$. Results: 67 pts were studied; 41 men and 26 women. The mdn age was 58 yrs. 42 had MBTs and 25 had PBTs. $30(45 \%)$ were randomized to AC Grp and $37(55 \%)$ to No AC Grp. $13(43 \%)$ of the AC group had primary BT and $17(57 \%)$ had metastases. Seizures occurred in $8(12 \%) ; 4(13.3 \%)$ in the AC Grp and 4 (10.8\%) in the No AC Grp; these were not different (Fischer's exact $\mathrm{p}=1.0$ ). These were GTC in $3(38 \%)$ and focal in $5(52 \%)$. Of the eight with Szs, two had subtherapeutic levels and three had progressive disease. AC Toxicity was seen in $2(7 \%)$ pts; one had rash and one increased LFTs. Compliance found $2(50 \%)$ with Szs had therapeutic serum levels; all others had a mdn 99\% (range, $26-230 \%$ ) therapeutic AC level. Mdn follow-up was 3.5 months. Conclusions: Seizures in BT patients were minor in some patients but serious in others. AC toxicities were minor. There was no differ- ence in $\mathrm{Sz}$ frequency between the AC Grp and No AC Grp. Continued accrual to increase statistical power and longer follow-up are needed to validate these observations.

\section{P-106}

\section{Epileptic Palinacousis in Malignant Astrocytoma}

A.M. Demchuk, P.A. Forsyth and D. Zochodne (Calgary, Alberta)

Palinacousis is a rare paroxysmal auditory illusion in which words or sounds recently heard persist or recur after the initial auditory stimulus has stopped. It usually reflects an underlying temporal lobe abnormality. Only two cases of palinacousis with malignant astrocytoma have been reported.

This 56-year-old left-handed, right hemisphere dominant man had two generalized seizures. EEG showed right temporal sharp waves and slowing; phenytoin was started. An MRI showed a temporal lobe mass and an anaplastic astrocytoma was removed. Three weeks later he had the acute onset of confusion and aphasia. An EEG revealed PLEDs arising from the right temporal lobe; the phenytoin level was subtherapeutic. His aphasia improved. On the third day he began "hearing echoes of peoples voices" in his left ear which were episodic. Words or short phrases just heard would persist and be repeated as "echoes" and last for several minutes. These echoes were specific for voice. As the phenytoin dose was increased the palinacousis became less frequent and was replaced by ringing in his left ear. A follow-up EEG showed less pronounced PLEDs.

Palinacousis may be a symptom of a brain tumour and requires investigation. It should not be confused with auditory hallucinations from dexamethasone-induced psychosis or psychiatric disease. Palinacousis and seizures are closely associated. The finding of PLEDs, the response to phenytoin, and the worsening of palinacousis during trough phenytoin levels, suggests this phenomenon may represent focal seizure activity. It can be treated successfully with anticonvulsants.

\section{P-107}

Primary T-Cell Lymphoma of the Brain Presenting as a Relapsing CNS Granulomatosis

Marc Petitclerc, Richard Desbiens, Daniel Lacerte and PETER-V. Gould (Quebec, Quebec)

Primary CNS T-cell lymphomas are distinctively rare. Most manifest as space occupying lesions, but some have features mimicking relapsing multifocal CNS disorders.

In 1987, a 65-year-old woman developed right sixth cranial nerve palsy which recovered spontaneously. In 1992, left sixth cranial nerve palsy, ataxia, fatique and pyramidal tract signs appeared. MRI revealed multiple small nodules, localized in the cortex, periventricular white matter, brain stem and cerebellum. Investigation was negative for multiple sclerosis, systemic tuberculosis, sarcoidosis, toxoplasmosis and neoplasia. Treatment with oral steroids resulted in clinical and radiological improvement. One year later, the patient was readmitted because of severe progressive ataxia and left-sided neglect. MRI showed a $2.5 \mathrm{~cm}$ right parietal lobe lesion. A stereotactic biopsy was performed and revealed changes consistent with primary T-cell lymphoma, with immunohistochemical staining positive for $\mathrm{T}$ cell markers MT2 and UCHL1. The patient was treated with chemotherapy and radiation therapy. The clinical picture stabilized (follow-up two months). 
Recent data including our patient suggest that primary CNS T-cell lymphoma may have a better prognosis than previously reported. Brain biopsy appears as the only reliable diagnostic tool. The clinical and radiological features of our case will be discussed and the literature will be reveiwed.

\section{P-108}

\section{Bromocriptine Induced CSF Leak; Case Report}

Eric Belanger, Daniel Lacerte and Michel Copty (Quebec, Quebec)

Prolactinoma is usually treated pharmacologically by the administration of Bromocriptine. Rarely, in the case of invasive adenomas, shrinkage of the tumour may be followed by cerebrospinal fluid (CSF) rhinorrhea. Such a complication has been reported to be managed surgically. We reoprt a case successfully treated by conservative therapy.

A 23-year-old man complained of headaches for about one year accompanied by hemianopsia, galactorrhea and visual impairment. Endocrinologic evaluation revealed a raised prolactin level: $3790 \mathrm{ug} / \mathrm{l}$ (normal: 0 - $10 \mathrm{ug} / \mathrm{l})$. A very large and invasive pituitary tumour was found on CT scan. Bromocriptine was initiated and five days later, a very active rhinorrhea clearly indication a CSF fistula appeared. A control CT scan was done and displayed an almost complete necrosis of the tumour and a defective superior sphenoidal sinus wall. With standard medical treatment including bed rest, head elevation and fluid restriction, the CSF fistula closed six days later and never recurred. The level of prolactine progressively dropped to a normal value and the patient's symptoms disappeared.

This is the first case of invasive microadenoma presenting a CSF leak induced by Bromocriptine and treated medically with success. Cases from the literature are briefly reviewed and management strategies are discussed.

\section{P-109}

\section{Transfection of a Human Fibrosarcoma Cell Line With a Glial Fibrillary Acidic Protein cDNA}

M. Murakami, S.L. Hubbard, K. Matsuzawa, K. Fukuyama, P. DIRKS and J.T. RUTKA (Toronto, Ontario)

Glial fibrillary acidic protein (GFAP) is an intermediate filament protein expressed almost exclusively by glial cells of the central nervous system. We have previously transfected GFAPnegative human astrocytoma cells with the gene for GFAP, and have demonstrated that GFAP transfection decreases astrocytoma proliferation and alters astrocytoma morphology. To determine if the same cellular responses could be elicited upon GFAP transfection of non-glial tumour cells, in the present study we have trasfected a GFAP-negative human fibrosarcoma cell line (HT-1080) with a cDNA containing the entire coding sequence of the human GFAP gene under the control of an inducible metallothionein promoter. Stably transfected HT-1080 clones were identified that are GFAP-positive by immunocytochemistry. GFAP-positive HT-1080 fibrosarcoma cells also demonstrate a decrease in tumour cell proliferation and altered morphological features when compared to controls. These findings suggest that the forced expression of the cytoskeletal protein, GFAP, can also be associated with dramatic cellular effects in non-glial, non-CNS tumour cells.

\section{P-110}

\section{Signal Transduction and Calmodulin Dependent Phosphodiesterase Activity in Brain Tumours}

S. Lal, R. Ragala and R.K. Sharma (Saskatoon, Saskatchewan)

Little or no progress has been made in the treatment of patients with malignant brain tumours. Disturbances of the components of the signal transducing systems could lead to uncontrolled cell proliferation and ultimately cancer. This may imply that malfunctions at the level of the receptors and or secend messengers may cause alteration in gene expression. The calmodulin dependent phosphodiesterase (PDE) is one of the most intensively studied and best characterized enzymes in the normal mammalian tissues, with very little known about this enzyme in tumour tissue. We have compared the activity of human brain PDE from normal cerebral tissue and various cerebral tumours. PDE activity isolated from various malignant tissue and from different regions of the brain was approx. tenfold lower than the corresponding activity observed in the control human brain tissue. We have also compared the activity of calmodulin in corresponding areas from normal and malignant brain tumours. An approx. threefold decrease in calmodulin activity was also observed in the malignant brain tumour as compared to the controls. In addition, the immunoblotting experiments suggested that PDE activity in highly malignant cerebral tumours failed to react with antibody, which was raised against bovine brain, whereas normal tissue reacted with the antibody. Although the significance of the observed study is not clear, it is noteworthy that the PDE activity in malignant brain tissue is lower than in normal brain tissue.

\section{P-111}

Expression of Cyclins, Cyclin Dependent Kinases, and Their Inhibitors in Astrocytomas by Northern Analysis, Immunoblotting, and Immunohistochemistry

Peter B. Dirks, James Rutka, Sherri lynn Hubbard, Masaji Murakami, Kazuhito Matsuzawa and Kozo fukuyama (Toronto, Ontario)

There is increasing evidence that perturbations in control of specific components of the molecular cell cycle machinery may be responsible for the uncontrolled growth of a cancer cell. As a result, we have studied the expression of cyclins, cyclin dependent kinases (CDKs), and cyclin dependent kinase inhibitors (CDKIs) in malignant human astrocytoma cell lines. Nine malignant human astrocytoma cell lines SF126, SF188, SF539, U87MG, U138MG, U251MG, U343MG-A, U373MG, and XF498 were studied by Northern blot analysis with human cyclin (A, B1, DI, D3, E) and CDK (CDK1, CDK2, CDK4, CDK6) and CDKI (p2l, p16) cDNA molecular probes. Immunohistochemistry and immunoblotting was performed with appropriate antibodies. Steady state cell cycle mRNA expression was analysed in unsynchronized tumour cells and in cells synchronized in GI phase to determine cell cycle 
phase specific gene expression. Analysis of steady state expression revealed increased levels of a variety of different cyclin transcripts and CDKs compared to normal brain or a primary culture of normal human fibroblasts. In several cell lines, there was increased expression of p21 mRNA in addition to increased cyclin and CDK expression. p16 transcripts were not identified in most cell lines. No aberrant transcripts were identified. Synchronized astrocytoma cells had appropriate timing of expression of cyclins. Immunohistochemistry revealed dramatic increased staining of astrocytoma cells for most cyclins and CDKs compared to human fibroblasts. Immunoblotting also revealed increased expression of protein in several astrocytoma cell lines with imperfect correlation to mRNA expression. Study is now being focused on those cell lines with overexpressed cyclins and p21 to better understand the molecular mechanisms of increased proliferation in these cells. We are also correlating expression of these genes between cell lines and primary tumours.

\section{P.112}

\section{Neurogenic Sarcomas: Experience at the University of Toronto}

Lily Angelov, A. Davis, B. O'Sullivan, R.S. Bell and A. GuHA (Toronto, Ontario)

Neurogenic sarcomas comprise $5 \%$ of all soft tissue sarcomas. In this study 11 patients with a neurogenic sarcoma managed at a regional sarcoma centre between $1989-1993$ were reviewed. Two patients had an underlying diagnosis of neurofibromatosis-1. 55\% presented with peripheral nerve symptoms associated with their mass. $73 \%$ of the tumours were Grade-3, $18 \%$ were Grade-2, and $9 \%$ were Grade-1 using the U.I.C.C. scale for grading sarcomas. The neurogenic sarcomas were also categorized according to size $(64 \%>5 \mathrm{~cm} ; 36 \%<5 \mathrm{~cm})$, a prognostic feature of soft tissue sarcomas.

At the University of Toronto Multidisciplinary Sarcoma Group, neurogenic sarcomas are managed similar to other soft tissue sarcomas, with local wide excision plus peri-operative radiation. In this review, with an overall median follow-up of 52 months, negative margins were achieved in six patients with continuous disease free status achieved in four of these patients. In the five patients resected with positive margins, none are continuously free of disease. In this latter group, four have suffered local relapse and one has systemic relapse. $80 \%$ of patients with local relapse eventually required limb amputation. This study thus suggests that wide resection with negative margins offers the best hope for cure as well as limb preservation in management of neurogenic sarcomas.

\section{P-113}

An Analysis of p53 and p16 Alterations in Primary Brain Tumours: Correlation With Changes in mRNA Expression

Eric Marmor, Jodi Lees, Elizabeth Sexsmith and David MALKIN (Toronto, Ontario)

Tumour suppressor genes are involved in the negative regulation of cellular proliferation. Both p53 and p16 (MTS1) are putative tumour suppressor genes that inhibit progression through the G1/S checkpoint of the cell cycle by binding with specific cyclin dependent kinasis. Both p53 and p16 have been found to be altered in a wide variety of malignancies, including glial cell tumours. There has been a very poor correlation between mutations detected at the DNA level and expression of abnormal protein products of the p53 gene. There have not been any studies to date examining this relationship for the p 16 gene. The purpose of this study is to examine the relationship between mRNA expression of these genes, and mutations at the DNA level in order to better understand their functional significance. Eight frozen tissue samples were obtained from glial cell tumours and genomic DNA as well as total RNA were extracted simultaneously using a $\mathrm{CsCl}$ technique. Single strand conformational polymorphism (SSCP) analysis was done on polymerase chain reaction (PCR) amplified DNA fragments for almost the entire coding region of both the p53 and pl6 genes. Reamplification, followed by sequencing was performed for the specific fragments that were found to have band shifts suggestive of mutations using SSCP analysis. Northern blot analysis of the RNA extracted from the tumours was accomplished with a probe specific for p53. 1/8 samples harboured a p53 mutation (H193R) while 3/8 samples show band shifts by SSCP for $p 16$ and are being currently confirmed. Interestingly the sample harbouring the p53 mutation was found to have markedly decreased RNA expression. These results suggest that both $\mathrm{p} 53$ and p16 play a role in glial cell tumour progression. Work is continuing on these samples as well as others to further investigate the role of tumour suppressor genes in the tumourigenesis of primary brain tumours.

\section{P-114}

Quantitation of ERCC-2 Gene Expression for Drug Resistance in Human Glioma Cell Lines by ReverseTranscription Polymerase Chain Reaction

Zhong-ping Chen, Susan Brien, Gerard Mohr and LaWrence PANASCl (Montreal, Quebec)

Background: Excision Repair Cross Complementing rodent repair deficiency (ERCC) genes are human genes implicated in nucleotide excision repair. ERCC-2 may be involved in recognition of DNA damage site and contribute to anti-cancer drug resistance. It is difficult to quantitate ERCC-2 gene expression in glioma specimens by Northern blot analysis because of its rarity. In order to correlate this gene expression with drug resistance in human glioma cells, we have developed a quantitative reverse-transcription polymerase chain reaction (RT-PCR) method. Methods: Total RNA were extracted from established glioma cell lines. After reverse transcription, cDNA was amplified by PCR obtaining a fragment of 617 bases. The cDNA was quantitated by a method using two separate tubes analysis. The ERCC-2 gene expression was calibrated with $\beta$-actin expression. Results: Our results show that glioma cell lines SKI- 1 and T98-G which are drug resistant have more than 10 folds ERCC2 gene expression than that of drug sensitive glioma cell line SK-MG-1. Our quantitative RT-PCR method can detect $0.000001 \mathrm{ng}$ ERCC-2 gene expression in $0.1 \mu \mathrm{g}$ total RNA sample, which is significantly more sensitive than Northern analysis. 
Ras Mediated Signal Transduction Pathway in Human Astrocytoma Cell Lines

\section{A. Guha, N. Lau and A. Pawson (Toronto, Ontario)}

Proliferation of human astrocytoma cells by activation of receptor tyrosine kinases (RTKs), such as Platelet-Derived Growth Factor (PDGF) and Epidermal Growth Factor (EGF) receptors, have been demonstrated. The ras signal transduction pathway, is utilized by many RTKs and its importance in proliferation of malignant human astrocytoma cell lines (U: 373 , $343,87,138$ ) was examined in this study. Immunoprecipitations and western blotting with antibodies directed at upstream activators of ras (SHc, GRB2, Syp), PDGF \& EGF RTKs and phosphotyrosine were utilized. The cells were transfected with the ras-Asn- 17 inhibitory mutant and effects on growth analyzed. Functional PDGF RTKs were expressed in 3/4 lines, while all lines expressed EGF RTKs. With EGF stimulation, the RTKs complexed with SHc, which was phosphorylated and bound $\mathrm{GRB}_{2}$. Syp was expressed by all lines and was constitutively phosphorylated in the U-138 cells. Induction of the ras-Asn-17 inhibitory mutant with either $\mathrm{Zn}^{+2}$ or dextramethasone in pooled or stable transfectants, decreased the number of viable colonies and proliferation rate in $3 / 4$ of the lines. These results demonstrate that mitogenic signals from RTKs in some human malignant astrocytoma cells utilize and depend on the ras pathway. Pharmacological methods of blocking ras signalling therefore may be of therapeutic use to decrease proliferation of human astrocytomas.

\section{EDUCATION $(P-116$ to $P-118)$}

\section{P.116}

Restructuring of the Neurology Residency Program Application Review Process at The University of Calgary

\section{N.A. HAGEN and L. METZ (Calgary, Alberta)}

Background: Beginning for the graduating medical school class of 1993, students could apply for neurology residency programs at the post-graduate year one (PGY-I) level through a computerized match service. For many Neurology programs, this resulted in a much greater number of applications to review. In this paper, we discuss the restructuring of the application review process over the past three years at The University of Calgary, and its outcome. Methods and Results: For a single PGY-1 position, each year an average of 34 applications were received. All applicants were invited to participate in a structured telephone interview. An average of eleven top ranked individuals were then evaluated during in-person interviews. Beginning for the 1995 graduating cohort, in-person interviews were highly structured, with each interviewer assigned specific questions. Conclusions: Structured telephone interviews for all applicants, and structured in-person interviews, were an efficient mechanism for a small residency program to evaluate a large number of applicants. The process of developing this interview system was found to be helpful to assist the selection committee to formulate its criteria for more effectively ranking a large number of applicants.

\section{Professional Liability of Canadian Neurologists and Neurosurgeons (A Comparative Study)}

\section{L.P. IVAN R.J. RoBson and W.J. BeIlby (Ottawa, Ontario)}

To define the present trends in neurological and neurosurgical malpractice lawsuits the Archives of the Canadian Medical Protective Association (CMPA) were studied covering 1979 1994. The data were analyzed statistically using simple descriptive statistics and comparative methods. Results: The Canadian experience shows:

I. an increase of certain medicolegal problems (college complaints, asking for advice)

II. a plateauing of the yearly incidence of lawsuits

III. a slowing down of the dramatic escalation of expenses

IV. a number of high risk areas which can be used for education.

There were significant differences between neurosurgeons and neurologists in:

I. the yearly incidence of lawsuits

II. the risk of being sued

III. the expenses of the defence

IV. the size of the payments awarded to the plaintiff by the courts

V. the proportion of lost and won lawsuits, and

VI. in some of the diagnostic and anatomical areas classified as high risk categories.

Conclusions: The malpractice profile of neurosurgeons is vastly different from the neurologists and from the rest of the medical profession. The difference in the magnitude of therapeutic risk may account for the difference in the incidence of lawsuits and the difference in expenses for legal protection. The incidence and outcome of lawsuits reached a steady state in the last five years; the possible reasons for this will be discussed.

\section{P-118}

The Use of Hypertext in the Development of Problem Based Neuropathology Tutorials

\section{C.R. SkINNER (Ottawa, Ontario)}

This demonstration will show a computer-based hypertext series of Problem Based Tutorials to enhance the teaching of neuropathology in a Problem Based curriculum.

Hypertext is a computer-based system of enrichment of text using "rich text format", colour imagery, video, and sound, which can be accessed through keyboard or mouse using a system of highlighting and hot words. The use of hypertext allows students to navigate through the learning material according to their learning requirements in a given subject area. The hypertext authoring system used for these modules allowed local content experts to author the course material into fully transportable Windows Help files which can be used in other centres. The ease of use of the authoring system is comparable to that of learning to use any Windows based word processor such as Microsoft Word or Wordperfect.

The demonstration will show modules already developed in ischemia, demyelination, neoplasia, and developmental disorders. 


\section{GENERAL NEUROLOGY $(P-119$ to $P-140)$}

\section{P-119}

\section{Dihydroergotamine and Metoclopramide in the Treatment of Organic Headache}

D.W. Gross, J.R. Donat, P. Davidson and C. Boyle (Saskatoon, Saskatchewan)

Dihydroergotamine (DHE) and metoclopramide have been used in the treatment of several types of benign headache for many years. The presumed mechanism of action of both DHE and metoclopramide is through the affinity these drugs have for serotonergic receptors. We present three cases of the use of DHE and metoclopramide in patients with headache due to organic pathology (two patients with viral meningitis and one with meningeal carcinomatosis). All three patients had excellent symptomatic relief. Our results demonstrate that DHE and metoclopramide can be effective in treating organic headache and, therefore, response to DHE and metoclopramide can not be assumed to signify benign disease.

\section{P-120}

The Experience of a General Internist in the Management of Chronic Daily Headache

Kevin P. Browne and Ashfaq Shuaib (Lethbridge, Alberta; Saskatoon, Saskatchewan)

Introduction: The management of patients with chronic daily headache had been relegated mostly to Neurologists practicing at teaching institutions. This paper presents the experience of a general internist attempting similar goals in a regional hospital. A total of 30 patients diagnosed with chronic daily headache were admitted electively for treatment to a secondary referral hospital (Lethbridge Regional Hospital, Alberta). The patients were from 11 to 72 years old (mean 39.5); mostly female (25); had a history of chronic headache for 1 to 50 years (mean 15.6); with a severity from 3 to 8 (mean 4.9) on a scale of $1-10$ and also had a history of migraine (26). They received intravenous Dihydroergotamine (DHE, Sandoz) 0.5 to $1.0 \mathrm{mg}$ every eight hours preceded by metoclopromide $10 \mathrm{mg} \mathrm{I.V.} \mathrm{and}$ amytriptyline $25-50 \mathrm{mg}$ PO at night. They continued subcutaneous DHE for an average of three days post discharge. Results: A complete resolution of headache was seen in $88.3 \%$ (25 of 30) with pain eliminated. Of the five non-responders, four were female, and three of the five went on to be diagnosed with psychiatric disorders. Conclusion: The management of appropriately selected patients suffering from chronic daily headache can be accomplished by an experienced internist in a NonUniversity setting with results that are comparable to published data.

\section{P-121}

A Newly-recognized, Acute Non-epidemic Encephalitis: Clinical, Neuroimaging, EEG and CSF Findings

A.O. OGUNYEMI (St. John's, Newfoundland)
Among the sporadic viral encephalitides, herpes simplex encephalitis is the best known. Over the last three years, we have observed eight patients with the clinical diagnosis of nonherpes "Viral" encephalitis all of whom show similar clinical, EEG and CSF features.

The patients' ages ranged from 16 to 75 years. Three were female and five male. The presenting clinical features were: acute alteration in the level of consciousness deteriorating to stupor or coma over 48 to 72 hours and low grade fever. The general medical examination was non-contributory. The neurological examination confirmed unresponsiveness without focal deficits.

CT scan of the brain was normal in all. The EEG findings ranged from generalized, high-amplitude delta slow waves with sleep spindles to intermittent generalized attenuation and burst suppression. The CSF showed elevated protein, normal glucose and mild lymphocytosis. Also, in the last five patients, polymerase chain reaction detected DNA of Epstein-Barr (EB) virus in the CSF.

All the patients became fully alert within three to five days with no neurological sequelae. One patient has had three attacks and five have had two attacks.

Additional studies are needed to fully characterize the disease and the etiologic agent.

\section{P-122}

\section{Viral Encephalitis: The Bug Bear of Neurology}

\section{B.M. Chaudhuri (Calgary, Alberta)}

Non-herpes simplex virus encephalitis is an uncommon cause of encephalopathy with an unknown etiology. In this poster, we will present three cases of undiagnosed encephalitis and the approaches to management of these patients and the outcome of their cases.

We have examined the differential diagnoses and the potential clinical pearls which can lead one down the path of a correct diagnoses. Also we will review the role of serological tests and brain biopsies in cases of localized lesions.

Finally, we will present a unique case of an immunocompetent, HIV negative patient who may have toxoplasmosis encephalopathy. An interesting aspect of his case is that treatment with sulfonamides may have caused him to develop bilateral hearing loss, a side effect never reported.

\section{P-123}

\section{Seronegative Toxoplasmosis Encephalitis in an AIDS Patient}

Zoe Arvanitakis, C. Power, L. Nocolle, J.N. Simonsen, A. NATH and A. Russell (Winnepeg, Manitoba)

Toxoplasmosis encephalitis (TE) is confirmed by positive toxoplasma gondii serology and neuroimaging demonstrating ring-enhancing lesions. A 41-year-old male with AIDS and a CD4 count of 14 (cells $/ \mathrm{mm}^{3}$ ), had an abrupt onset of dysarthria and ataxia associated with a "flu-like" illness. He demonstrated psychomotor slowing, dysdiadochokinesia and was unable to walk unassisted. Cranial CT scans, with and without contrast, were initially reported as normal but subsequent review demonstrated one brainstem and two periventricular non-enhancing 
hypodensities. Serum IgM and IgG antibodies to toxoplasmosis were undetectable at admission. CSF studies revealed 5 cells $/ \mathrm{mm}^{3}$ ( $100 \%$ lymphocytes), normal cytology, glucose $(3.5$ $\mathrm{mmol} / \mathrm{l})$ and cultures for bacteria, viruses and fungi were negative. CSF protein was elevated $(1.27 \mathrm{~g} / \mathrm{L})$ and the CSF immunoelectrophoresis showed two bands. MRI with gadolinium showed 17 enhancing lesions in grey and white matter. He was started on pyramethamine, sulfadiazine, and folinic acid. He improved over the next four weeks, and, discharge, his speech was clear and he was able to walk unassisted. He has remained toxoplasma seronegative since discharge. The clinical and MRI features and response to therapy indicate that he had TE. The negative toxoplasma serology which may reflect a depressed humoral response in an immunocompromised host and the absence of enhancing lesions on CT scan is an unusual presentation of TE. This case illustrates the lack of sensitivity in detection of TE with CT scanning and toxoplasma serology. MRI should be used where there is a high index of suspicion for this diagnosis in the face of other tests being inconclusive.

\section{P.124}

\section{Enkephalitis Precipitated by Systemic Treatment of Filariasis With Diethylcarbamazine}

Manon Thibault, J.V. Villeneuve, S.T. Trottier and J.-P. BouCHARD (Quebec, Sainte-Foy, Quebec)

The filarial parasites of humans infect over 100 million people. Wuchereria bancrofti alone is worldwide in the tropics and responsible for three-quarters of the cases. Case report: A 27 year-old black male native of Zaïre, in Quebec for three years, complained of fever, fatigue, headache, arthralgia, diffuse pruritus, coughing and abdominal pain. Physical and neurological examination were normal. Blood tests showed filariae of the Wuchereria bancroffi type. The patient was started on diethylcarbamazine (DEC). He was admitted a week later for severe confusion. He was alert but disorientated and dysphasic, without nuchal rigidity. CSF was within normal limits, as well as CT and MRI scans. EEG showed a moderately severe diffuse slowing more marked over the left frontal region, potentially epileptogenic. The patient was treated with Acyclovir and Phenytoin and became drowsy and weak in the right hand. Decadron was started the next day. Although cell count and proteins remained normal, microfilariae were identified in CSF. Acyclovir was stopped; DEC and Decadron were continued. The patient was normal on discharge one month later. Conclusion: Enkephalitis is a wellknown severe and often fatal complication of DEC treatment. The prophylactic use of steroids seems to be of definite value in such cases.

\section{P-125}

\section{Auditory Hallucinations Secondary to Listeria Brainstem Abscess}

\section{A.G. Douen and P.R. Bourque (Ottawa, Ontario)}

Complex auditory hallucinations occur in neuropsychiatric disorders and lesions of the temporal lobe or peripheral auditory system. A few cases were described with acute pontine lesions, mostly hemorrhages. We report an association with a brainstem abscess.

A 43-year-old man presented with a prodrome of headache, fever, and vomiting. He developed nystagmus, ataxia, and progressive left-sided cranial neuropathies V, VII, VIII, IX, X. The initial CT was normal but the CSF was suggestive of meningitis and Listeria Monocytogenes was identified in blood cultures. The patient developed nearly complete hearing loss on the left and musical auditory hallucinations. While fully alert, he reported hearing "big band music" and specific songs, in particular "Summer Girl". A series of CT scans and MRI showed an evolving pontine abscess. With antibiotic treatment, there was initial improvement, then deterioration with a new left hemiparesis. Imaging studies at that stage were consistent with hemorrhage into the large pontine abscess and surgical decompression was successfully carried out.

To our knowledge, musical auditory hallucinations have not been previously described in the context of rhombencephalitis. The likely pathogenesis is a release phenomenon due to involvement of central audotory pathways (acoustic striae, trapezoid body, superior olivary nucleus).

\section{P-126}

Familial Prion Disease: Phenotypic Variability, Diagnostic Difficulties, and Diagnostic Approach

Andrew M. Demchuk, Carey Johnson and Gary Klein (Calgary, Alberta)

An autosomal dominant pattern of inheritance has been recently described in the prion diseases. Creutzfeldt-Jakob (CJD), Gerstmann-Straussler-Scheinker, and Fatal Familial Insomnia are linked to germline mutations in the coding region of a single gene, prion protein gene (PRP) on chromosome 20 . We describe two families with clinical, pathologic and genetic evidence confirming familial Creutzfeldt-Jakob disease.

Family 1 consists of a father with early dementia and two daughters who have clinically, and pathologically confirmed CJD. Family 2 consists of a mother with a clinical history suggestive of fatal familial insomnia and two sons with pathologically confirmed CJD.

Fatal Familial Insomnia and Creutzfeldt-Jakob disease have never been reported in the same family. Both diseases can have mutations at codon 178 of PRP gene. A methionine-valine polymorphism at codon 129 determines which phenotypic expression FFI or CJD is present.

These kindreds illustrate the phenotypic variability, potential diagnostic difficulties, and diagnostic approach of familial prion disease.

Progressive Subcortical Dementia With Alien Limb Syndrome. A Variant of Cortico-basal Ganglionic Degeneration?

OsCar Benavente, E. Mohr and C.L. Barclay (Ottawa, Ontario)

Alien limb phenomenon (ALP) has been described in association with stroke, lesions of the corpus callosum and callosectomy. 
However, in its pure form it has been associated with only one neurodegenerative disorder, cortico-basal ganglionic degeneration (CBGD). We present a case of ALP associated with a neurodegenerative disorder lacking other features of CBGD.

This 68-year-old man had a two year history of headache, progressive dementia and difficulty using his left arm. The arm "had a mind of its own" and unless held down, it would move involuntarily. Examination showed impaired saccades, paratonia, semi-purposeful movements in the left arm, and left-sided apraxia with prominent grasp response. No parkinsonism, stimulus-sensitive myoclonus or cortical sensory loss were present. MRI was unremarkable, SPECT scanning demonstrated hypoperfusion of the right parieto-temporal region and brain biopsy of that region was normal except for mild congophilic angiopathy.

This case illustrates that progressive ALP can occur in absence of other features of CBGD. With negative brain biopsy, post-mortem examination will be required to determine if this case is due to a variant of CBGD or another neurodegenerative disorder not previously known to be associated with ALP.

\section{P-128}

\section{Dementia and Normal Aging: a Comparative} Neurobehavioural Assessment

\section{S. Darvesh, M. Freedman, L. Leach, S. Black and E. Kaplan} (Halifax, Nova Scotia; Toronto, Ontario)

An appreciation of the patterns of cognitive impairment is important for diagnosis, management and future research in patients with cognitive decline. Various mental status examinations are available to physicians for use in the office or at the bedside but none are comprehensive and concise. To fill this gap we devised the "Toronto Baycrest Behavioural Neurology Assessment" (BTBNA), which briefly assesses salient cognitive domains: level of awareness, physical appearance, emotional state, attention, memory, language, visuospatial function, manipulation of acquired knowledge, frontal lobe function and praxis. To obtain normative data, the BTBNA was administered, along with the Mini-Mental State Examination (MMSE) to 123 subjects. To evaluate the effectiveness of BTBNA in identifying cognitive impairment, the results obtained from subjects in the normative study were compared to those obtained from patients with dementia of mixed etiology ( $\mathrm{N}=29 ; 15$ females, 14 males in each group). The two groups differed significantly on the total MMSE scores as well measures of all cognitive domains assessed in BTBNA. However, BTBNA was more sensitive at identifying impairments in several areas including language (naming) and visuospatial function (clock drawing and cube copying). Our preliminary results suggest that the BTBNA provides a concise, comprehensive and sensitive overview of mental status functions in the clinical setting.

\section{P-129}

Intranasal Administration of Nerve Growth Factor in the Rat and in Patients With Alzheimer's Disease

J.D. Turnbull, F. Jiang, W. Malloy and D. Muir (Hamilton, Ontario)

Alzheimer's disease (AD) is characterised by cholinergic cell loss in the deep septal region. Cholinergic cells are trophically supported by NGF, and administration of NGF reduces septal cell loss in aged rats and in fimbrectomized young rats. In the absence of suitable animal models, growth factor therapy for $\mathrm{AD}$ has been supported as an acceptable human research initiative, and two patients in Sweden have received NGF via intraventricular infusion with some promise. However intraventricular infusions are problematic, as are intravenous injections, and we are exploring alternative modes of delivery. Wheat germ agglutinin and colloidal gold are taken up by nasal epithelium and transported trans-synaptically to areas of the brain with anatomical connections with the olfactory system, including those heavily involved in Alzheimer's disease. We have argued that the nasal route might be an appropriate route for the administration of neurotrophins to the brain in $A D$, and for some time have been investigating the feasibility of administering nerve growth factor alone, or in biodegradable microspheres to enhance pinocytosis and trans-synaptic spread. We will discuss the rationale, results in rats, and preliminary results of a feasibility trial in patients with advanced AD.

\section{P-130}

\section{Anticardiolipin Antibodies in Women With Silicone Breast Implants}

Britta Ostermeyer Shoaib, Bernard M. Patten, Dale SAlAZAR and GlenN ARmEN (Houston, Texas)

We started to measure anticardiolipin antibodies in women with silicone breast implants who were referred to us for evaluation of neurological disease. Measurements were done by indirect immunofluorescence. 42 of 200 women were found to have anticardiolipin antibodies (26 IgG, $12 \mathrm{IgM}, 4$ both IgG and IgM). Mean latency period between implant surgery and development of symptoms was 5.8 years (range 3 months -15 years). 38 had polyneuropathy and four had an MS-like syndrome. Cerebellar findings were seen in 16/34. Other symptoms included myalgia, arthralgia, joint stiffness, memory problems, vision problems, skin rashes, dry eyes and mouth, Raynaud's phenomena and TIAs. Decreased blood perfusion on SPECT scan was seen in 23/35, MRI showed white matter lesions in 12/37, EKG showed $T$ waves in 13/36 and sural nerve biopsies showed loss of myelinated fibers in 25/27. Anticardiolipin antibodies should be added to the list of abnormal laboratory findings in patients with breast implants and neurological disease.

\section{P-131}

\section{Unifying Metrics for Normal Human Saccades}

\section{J.J.S. BARTON (Toronto, Ontario)}

Previous studies have used power, exponential, and inverse linear functions empirically to relate saccadic peak velocity to amplitude. However, I show that this curve can be predicted solely from the parameters of the equations for the duration/amplitude and peak-velocity/mean-velocity relationships, rendering prior empiric equations unnecessary. 
I studied 2808 saccades from four normal subjects. Compared with previous empiric curves, the derived equation had less error and did not underestimate the peak velocity of small saccades. It also showed that saccadic metrics are not unitary but vary at different amplitudes. Between $5^{\circ}$ and $25^{\circ}$ centrifugal and centripetal saccades have similar linear duration/amplitude and peak-velocity/ mean-velocity functions. Below $5^{\circ}$ increases in saccadic amplitude are accompanied by smaller increments in peak velocity and larger increments in duration. Above $25^{\circ}$ centrifugal but not centripetal saccades are slower and longer, likely due to orbital mechanics. These variations are obscured when the previous empiric equations are used. Lastly, the peak-velocity/meanvelocity ratio is not constant, but declines at small amplitudes, a finding also predicted from the duration/amplitude and peakvelocity/mean-velocity relationships.

\section{P.132}

Demonstration of an Extra-retinal Pursuit Signal in Human Lateral Occipital Cortex With Functional Magnetic Resonance Imaging

J.J.S. Barton, E. Kiriakopoulos, C. Stewart, K. Davis, D. MIKULIS and T. SIMPSON (Hamilton, Toronto, Waterloo, Ontario)

We imaged seven normal human subjects with a conventional 1.5 Tesla MR instrument, using the following parameters: SPGR gradient echo, TR $68 \mathrm{~ms}$, TE $40 \mathrm{~ms}$, matrix $256 \times 128$, field of view $30 \times 22 \mathrm{~cm}$, flip angle $45^{\circ}$, and slice thickness of $4 \mathrm{~mm}$. Subjects watched either a moving grating with their gaze fixed on a stationary target, or pursued a small moving spot. During the control period they stared at a stationary grating. We obtained six images during each of the three condition periods, and repeated the cycle six times, for a total of 108 images. Activation was identified using a pixel-by-pixel t-test analysis comparing mean signal intensity in one condition with that of another.

In lateral occipital cortex, a region previously identified as a human homologue of V5, a significant increase in signal intensity was seen in five subjects. This increase was greater during dot pursuit than viewing of the moving grating, despite the greater retinal slip created by the grating. This implies activation related to the act of pursuit, rather than the visual motion of the target. Signal in V1 and V2 decreased during pursuit, consistent with the lesser visual stimulation.

These results suggest that, in addition to a homologue of V5, a homologue of the monkey's medial superior temporal area (MST) exists in the lateral occipital cortex of humans.

\section{P-133}

\section{Oxygen Free Radicals and Alzheimer Disease}

S. Abo El-Ghar, A.H. Rajput, S.V. Mantha, J. Kalra and K. PRASAD (Saskatoon, Saskatchewan)

Alzheimer's disease (AD) is a neurodegenerative disorder. Degenerative changes in the brain of $A D$ could be due to increased levels of cytotoxic oxygen free radicals (OFRs) because of its increased production and/or decreased destruction. We, therefore, investigated the OFR-producing activity of polymorphonuclear leukocytes (PMNL-CL), lipid peroxidation product malondialdehyde (MDA), activated PMNLS (NBT positive PMNLs), and antioxidant enzymes (superoxide dismutase, catalase, glutathione peroxidase) in blood from 15 age matched healthy subjects and patients with AD. There was an increase in the number of activated PMN leukocyte, the activity of antioxidant enzymes, and plasma MDA and a decrease in oxygenderived and SOD-inhibitable PMNL-CL in patients with AD. Increased release of OFRs from PMNLs is suggested by decreased PMN-CL and increased number of NBT positive PMNLs. In MDA inspite of increased antioxidant enzymes suggests increased levels of OFRs in excess of the capacity of antioxidants to scavenge OFRs. These results suggest that there is an increased level of OFRs, and that PMNLs may be one source of OFR. OFRs may, therefore, be involved in the pathophysiology of Alzheimer's disease.

\section{P-134}

Clinical Course and Sequential Neuroimaging in a Pattern With Severe Delayed Carbon Monoxide (CO) Toxicity (Grinker's Myelinopathy)

\section{B.M. ChaudhurI (Calgary, Alberta)}

This case of a 59-year-old male with Grinker's Myelinopathy demonstrates remarkable clinical recovery and the associated sequential imaging changes beginning with $\mathrm{CT}$ one year prior to $\mathrm{CO}$ exposure. Although many cases of delayed $\mathrm{CO}$ toxicity have been previously reported this is the first to report the disease course in detail.

The patient suffered acute $\mathrm{CO}$ toxicity after falling asleep in his running car. Several hours later he was discovered comatose; the car was no longer running. In the emergency department his carboxyhemoglobin level was $11 \%$. He was admitted and treated with normobaric oxygen. He recovered rapidly and was back at work within two weeks.

Three weeks after the initial exposure he began to rapidly deteriorate. He developed severe dementia, incontinence and parkinsonism. His deterioration continued for two weeks, he remained stable for three weeks, and he is still recovering. At his most disabled he could not speak or move. Trials of Ldopa/carbidopa and bromocriptine were useless. He received extensive rehabilitation therapy and is again living independently although he is not employed due to residual high level cognitive deficits.

Within (16) hours of $\mathrm{CO}$ exposure a CT scan demonstrated hypodensities in the globus pallidus bilaterally consistent with $\mathrm{CO}$ exposure. (Such lesions have not previously been reported so soon after exposure but with the previously normal scan we believe these lesions were due to this poisoning). MRI scans over one month demonstrated white matter changes.

\section{P-135}

\section{Executive Dysfunction in Simultanagnosia}

S. Naidj, Michel Panisset and Martine Simard (Montreal, Quebec)

Objective: To clarify the role of attentional deficit in simultanagnosia in Balint syndrome. Background: Balint syndrome consists in optic ataxia, gaze apraxia, and simultanagnosia. 
The nature of simultanagnosia is still unclear. Design/Method: A 63-year-old woman with a one year history of progressive visual disturbance had optic ataxia, gaze apraxia, and simultanagnosia on exam. Among tests of a comprehensive neuropsychological battery, cancellation tasks were designed in order to test attentional functioning in the context of a severe visuospatial (VS) handicap. Either pre-ordered arrays $(\mathrm{O})$ or randomly organized (R) stimuli were presented. In the first condition (1), a single stimulus had to be crossed whereas in the second one (2), two stimuli had to be crossed always in the same order. Results: Simultanagnosia was evident on VS recognition tests. There were $5 / 12$ omissions in condition $\mathrm{O} 1$ and $8 / 13$ in condition $\mathrm{O} 2$, $8 / 12$ omissions in condition $\mathrm{R} 1$ and $11 / 13$ in condition R2. Conclusion: The poor performances on tasks minimally demanding on the working memory (conditions $\mathrm{O} 2$ and R2) supports that simultanagnosia may at least in part be explained by an attentional system deficit.

\section{P.136}

Neuroradiological Correlates of Neuropsychological Disorders in Olivopontocerebellar Atrophy (OPCA)

T. Botez-Marquard and Olga-Lucia Pedraza-Linares (Montreal, Quebec; Sante Fe de Bogota, Colombia)

Thirty-two OPCA patients without cortical atrophy were studied. Radiological data were correlated with neuropsychological evaluations on the following five neuroradiological measures: subjective assessment of cerebellar atrophy with estimation of the brainstem ratio, midbrain ratio, fourth ventricular ratio and brachium pontis ratio. From the radiological point of view, the patients were divided in two groups: one with mild, and the other with moderate-severe atrophies. The neuropsychological assessment measures were: global memory quotient, verbal learning capacities, recall and recognition, attention, abstract thinking, visio-spatial and visuo-constructive functioning. In general, patients with moderate-severe atrophies showed more marked cognitive impairment than those with mild atrophies.

These results suggest that neuropsychological deficits in OPCA patients are mainly related to the neocerebellum and to neocerebellar cortical-cerebellar loops.

\section{P-137}

\section{Reversible Focal Encephalopathy Sequel to Remote Brain Irradiation}

\section{J. ScotT and A. OGunYemi (St. John's Newfoundland)}

Focal cerebral necrosis and diffuse cerebral injury are well known delayed complications of brain irradiation. We report three patients with reversible, acute focal encephalopathy occurring between 8 and 14 years after brain irradiation administered for the treatment of primary brain tumours.

For the three patients, the presenting clinical features were: (1) low grade fever, (11) headache, (111) obtundation and (IV) focal neurological deficits which were unrelated to the sites of their brain tumours. One patient also had a generalized seizure.

In addition, the neurological examination revealed left hemiparesis, left tactile neglect and left homonymous hemianopia in one patient, the second patient had right homonymous hemianopia, right hemiparesis and aphasia while the third showed aphasia and right hemiplegia. Also, all the three patients had nystagmus and gait ataxia.

CT scan showed only changes localized to the site of the primary brain tumours in all the patients. The EEG however, demonstrated focal abnormalities correlating with the focal cerebral deficits. There was complete resolution of the acute neurological deficits within one to two weeks, in the three patients.

The possible mechanisms of the acute focal encephalopathy will be discussed.

\section{P-138}

\section{Withdrawn}

An Uncommon Phenotypic Expression: Adult Cerebral Adrenoleukodystrophy

Donald Rivest, Ariane Mackey, Jean-Pierre Bouchard and Manon Thibault (Quebec, Quebec)

Phenotypic expression of X-linked adrenoleukodystrophy is highly variable. Only a few cases of the adult cerebral form have been described. A 44-year-old man known for "probable multiple sclerosis" was admitted for a first seizure. At age 39 he developed progressive gait disturbance. One year prior to admission, personality changes, abnormal behaviour and intellectual decline were noticed at work and by his family. 
Medical history revealed multiple infections with unexpected lengthy recovery. Family history was unrevealing except for a nephew who had "dark skin". The patient had melanodermia and was almost bald. Neurologic examination revealed disinhibited behaviour, profound cognitive impairment, mild ataxia, brisk deep tendon reflexes and bilateral Babinski signs. Speech, visual acuity and hearing were normal. There was no weakness, nor sensory loss. MRI showed extensive symmetric white matter lesions in the frontal and posterior parietal regions sparing the U-fibers. Nerve conduction studies were normal. Plasma very long chain fatty acids ratio (C-26/C22) was increased fivefold and mild adrenocortical insufficiency was documented.

Adult cerebral adrenoleukodystrophy (ACALD) has a variable clinical expression and might be underdiagnosed. Since family screening is mandatory for genetic counselling, diagnosis of ACALD should not be overlooked. Treatment options might be available for asymptomatic patients.

\section{P-140}

Prevalence of Vitamin $B_{12}$ Deficiency and Thyroid Abnormalities in Dementia

A. Krajewski, J. Springer, J. Jones, T. Xerri and M. Freedman (North York, Toronto, Ontario)

Objective: Prevalence of Vitamin $B_{12}$ deficiency and thyroid abnormalities associated with Alzheimer's disease (AD), Parkinson's disease (PD) and multi-infarct dementia (MID). Design: Retrospective study of consecutive patients. Setting: Behavioural Neurology Assessment Unit. Subjects: 251 consecutive patients; male/female $=112 / 139$; mean age $71 \pm 11$ years. Results: Although $145(63.2 \%)$ patients had AD, only 115 had $\mathrm{AD}$ as a single diagnosis. $25(10.8 \%)$ patients had $\mathrm{PD}$ with 16 having PD only. 26 (11.3\%) subjects had MID with 16 having MID only. Prevalence rate for patients with a single diagnosis of AD, PD and MID for vitamin $B_{12}$ deficiency was $7 \%, 12.5 \%$ and $6.25 \%$ respectively. Prevalence rate of elevated TSH was $4.3 \%$ in $\mathrm{AD}, 0 \%$ in $\mathrm{PD}$ and $12.5 \%$ in MID. Differences between proportions of vitamin $B_{12}$ abnormalities in $A D, P D$ and MID were not significant. Differences between proportions of TSH abnormalities in AD and MID were not significant. Also there was no relation between blood test results and diagnosis. Conclusions: The prevalence rates of vitamin $\mathrm{B}_{12}$ and TSH abnormalities in our series of patients with dementia suggest that vitamin $B_{12}$ deficiency and elevated TSH may be important contributors to the congnitive impairment in AD, PD and MID. The follow-up of treated patients is required to further address this issue.

\section{SPINAL CORD $(P-141$ to $P$ - $/ 45)$}

\section{P-141}

Failure of Intrathecal Nimodipine to Increase Spinal Cord Blood Flow in the Normal and the Injured Rats

\section{H. IMAMURA and C.H. TATOR (Toronto, Ontario)}

We have previously demonstrated that the intravenously administered calcium channel antagonist nimodipine produced an increase in spinal cord blood flow (SCBF) in normal and injured rats. However, in the injured rats, due to the hypotension caused by both cord trauma and the vasodilator effects of nimodipine, it was necessary to counteract the hypotension with volume expansion or vasopressors to maintain posttraumatic arterial blood pressure. The present study was performed to investigate whether intrathecally infused nimodipine could increase SCBF in normal and injured rats.

Male Wistar rats anesthetized by $\alpha$-chloralose and urethane had a laminectomy from $\mathrm{Cl}$ to $\mathrm{TI}$ and then a silastic tube was inserted into the subarachnoid space via the atlantooccipital membrane and passed to the $\mathrm{C} 6$ level. We administered intrathecal nimodipine $(0.05 \mathrm{mg} / \mathrm{kg}, \mathrm{n}=5 ; 0.2 \mathrm{mg} / \mathrm{kg}, \mathrm{n}=5)$ or placebo $(\mathrm{n}=5$ ) to normal rats, and measured SCBF at C7/T1 with the hydrogen clearance method before infusion, during infusion and 30 minutes after infusion of the drug or placebo. Neither 0.05 nor $0.2 \mathrm{mg} / \mathrm{kg}$ of nimodipine increased SCBF during infusion $(p>0.05)$ or after infusion ( $p>0.05$ ). We also administered intrathecal nimodipine $(0.05 \mathrm{mg} / \mathrm{kg}, \mathrm{n}=5)$ or placebo $(\mathrm{n}=5)$ to rats injured by acute compression with a $35 \mathrm{~g}$ clip for one min. There was no improvement in posttraumatic spinal cord ischemia $(p>0.05)$ or axonal dysfunction $(p>0.05$ ) assessed by somatosensory and cerebellar evoked potentials. These results show that intrathecal nimodipine at these doses was not beneficial for the normal or injured spinal cord.

\section{P-142}

\section{Cauda Equina Syndrome Following Spinal Anesthesia}

Ziad Nasreddine and Jean-Pierre Bernier (Sherbrooke, Quebec)

Cauda Equina Syndrome (CES) is a rare but serious complication associated with spinal anesthesia. Drasner (Anesthesiology 92) and Cheng (Anesth. Analg 94) described two patients with CES following subarachnoid administration of $2 \%$ lidocaine. We report a patient with CES following spinal anesthesia.

A 73-year-old male patient, presented with urinary retention and urologic examination revealed prostatic hypertrophy. The patient underwent transurethral resection under spinal anesthesia. Lidocaine $5 \%$ (75 $\mathrm{mg}$ without epinephrine) was administered in the subarachnoid space at the $\mathrm{L}_{3}-\mathrm{L}_{4}$ level and a $\mathrm{T}_{6}-\mathrm{T}_{7}$ analgesic level was obtained. Immediately following the operation the patient complained of fecal incontinence and neurologic examination showed bilateral weakness in the $L_{5}-S_{1}$ territory with an anesthesia in a saddle-like distribution, and abolished ankle reflexes. Myelography and CT-Scanning did not show any lesions in the spinal canal. Detailed electrophysiological studies will be presented. Patient status remained unchanged two months after surgery.

Possible mechanisms include neurotoxic and/or ischemic effects on spinal nerves caused by large doses of lidocaine with or without epinephrine.

Further experience will be necessary to elucidate the mechanism of injury in this serious but fortunately rare complication. 


\section{P-143}

\section{Listeria Spinal Cord Abscess - Clinical Features and MRI Findings}

J.Y. Chu, W. Montanera and R.A. Willinsky (Toronto, Ontario)

Intramedullary spinal cord abscess due to Listeria Monocytogenes is an uncommon condition usually affecting immunocompromised patients. The following case report illustrates the importance of serial MRI to determine the degree of response following antibiotic therapy.

A 69-year-old man presented with three weeks history of subacute onset of lower limbs paraparesis and left upper limb weakness. Myelogram and CT scan showed widened upper cervical cord. CSF revealed lymphocytosis, moderately elevated protein and depressed glucose. A Gadolinium enhanced MRI showed diffuse cervical cord edema with two ring-enhancing lesions at C2-3. Blood and CSF cultures grew Listeria Monocytogenes. He received IV Ampicillin and Gentamycin, the latter was discontinued after one month due to nephrotoxicity. Serial MRI over the next three months showed significant reduction in the size of these abscesses. The patient demonstrated modest improvement in the power of his lower limbs, however, he remained bedridden. Aside from being a mild, diet-controlled diabetic, there was no other evidence of immuo-suppression and he was HIV negative.

The clinical features and serial MRI findings will be presented in detail. A Medline search (1980 - 1994) shows no previous report utilizing MRI to serially image Listeria spinal cord abscess.

\section{P.144}

Spinal Cord Stimulation is the Treatment of Choice in Refractory Reflex Sympathetic Dystrophy: a 10-year Experience

\section{K. KUMAR and R. NaTH (Regina, Saskatchewan)}

An analysis of 228 patients receiving spinal cord stimulation (SCS) revealed 18 patients diagnosed with Reflex Sympathetic
Dystrophy (RSD). The mean age of the 13 men and five women was 42.1 years. All suffered limb injuries, from a variety of causes.

At an average of 46 months follow-up, 12 patients were using their stimulators regularly and only two were using minor pain medication. The level of pain control was determined by administering a modified McGill Pain Questionnaire.

Seven patients reported excellent, three good, and two fair pain relief. Six patients reported no pain relief; these six had pain of mixed etiology from severe multicompartment injuries which had responded poorly to surgical sympathectomy. Five minor hardware related complications occurred.

The low morbidity of SCS and its efficacy in patients failing surgical sympathectomy suggest that SCS is logically superior to ablative sympatholysis in the management algorithm of reflex sympathetic dystrophy. RSD patients with additional sources of pain do not respond well to SCS.

\section{P-145}

\section{Transient Weakness of Both Arms. Spinal Cord T.I.A?}

\section{C.A. SIMPSON (Victoria, British Columbia)}

The case histories of three patients are presented who have had several episodes of brief transient weakness of both arms simultaneously without involvement of the legs. Two of them have evidence of cardiac ischemia but in none of them has the episode ever been precipitated by exertion. All three patients have cervical spondylosis, two significantly so. All have had magnetic resonance imaging of the cervical cord and one had had cervical discectomy and fusion. In none was there a history of trauma precipitating the episode, but there may be some association with neck position.

No previous description of a similar syndrome could be found. Something similar but more persistent can be seen with the "central cord syndrome". It is postulated that the syndrome may be caused by impairment of blood flow to the cord by compression or irritation of the radicular arteries resulting in either temporary occlusion or spasm. In these three patients the syndrome appears to be benign. The main differential diagnosis is angina but reasons are given why this is probably not angina. 